\title{
GALOIS ACTION ON KNOTS II: PROALGEBRAIC STRING LINKS AND KNOTS
}

\author{
HIDEKAZU FURUSHO
}

\begin{abstract}
We discuss an action of the Grothendieck-Teichmüller proalgebraic group on the linear span of proalgebraic tangles, oriented tangles completed by a filtration of Vassiliev. The action yields a motivic structure on tangles. We derive distinguished properties of the action particularly on proalgebraic string links and on proalgebraic knots which can not be observed in the action on proalgebraic braids. By exploiting the properties, we explicitly calculate the inverse image of the trivial (the chordless) chord diagram under the Kontsevich isomorphism.
\end{abstract}

\section{INTRODUCTION}

This paper is a continuation of our previous paper [F6, where the action of the absolute Galois group on profinite knots was constructed by an action of the Grothendieck-Teichmüller profinite group $\widehat{G T}$ there. While in this paper, the action of the motivic Galois group, which is the Galois group of the tannakian category of mixed Tate motives over Spec $\mathbb{Z}$, on the linear span of proalgebraic tangles is deduced from an action of the Grothendieck-Teichmüller proalgebraic group $G T(\mathbb{K})$ $(\mathbb{K}$ : a field of characteristic 0$)$ there.

Proalgebraic tangles (Definition 2.3) means the $\mathbb{K}$-linear span of oriented tangles completed by a filtration à la Vassiliev. Proalgebraic n-string links (resp. proalgebraic knots) are proalgebraic analogues of $n$-string links (a good example can be found in Figure 2.1) (resp. knots) and they are subspaces of proalgebraic tangles spanned by them. In $₫ 2$ we give a $G T(\mathbb{K})$-action on proalgebraic tangles by following a method indicated in $[\mathrm{KT}$. The action is interpreted as an extension of the $G T(\mathbb{K})$-action on the proalgebraic braids (reviewed in $\S \mathbb{1}$ ) into the one on the proalgebraic tangles. In 33 we derive distinguished properties of the $G T(\mathbb{K})$-action on proalgebraic tangles which can not be observed in $G T(\mathbb{K})$-action on proalgebraic braids. Particularly

Theorem A (Theorem 3.14 and Proposition 3.19). Let $G T_{1}(\mathbb{K})$ be the unipotent part of $G T(\mathbb{K})$. Then

(1) The $G T_{1}(\mathbb{K})$-action on proalgebraic string links is given by an inner conjugation.

(2) The $G T_{1}(\mathbb{K})$-action on proalgebraic knots is trivial, which yields a non-trivial decomposition (3.16) of each oriented knot.

They are derived by Twistor Lemma (Lemma 3.2 and 3.12), which can be seen as reformulations of AT2 Theorem 7.5, AET Theorem 2, LM1 Theorem 8 and [LS] Theorem 2.1 in our setting, though all of which originate from [Dr] Theorem

Date: August 21, 2017.

2010 Mathematics Subject Classification. Primary 16W25; Secondary 11M32, 20F36, 57M25. 
$\mathrm{A}^{\prime}$. The action on proalgebraic $n$-string links in Theorem A.(1) is faithful for $n \geqslant 3$ and is not faithful (actually it is trivial) for $n=1$ (see Remark [3.16). However the case for $n=2$ remains unsolved (Problem 3.17). Since $G T(\mathbb{K})$ contains the motivic Galois group, our $G T(\mathbb{K})$-action on proalgebraic tangles yields the Galois action there, which yields a structure of mixed Tate motives of Spec $\mathbb{Z}$ there. Particularly Theorem A.(2) says that proalgebraic knots can be decomposed into infinite summations of Tate motives.

By exploiting the properties shown in the above theorem, we explicitly determine the inverse image of the unit $e$, the chordless chord diagram on the oriented circle, under Kontsevich isomorphism $I$ in (2.33):

Theorem B (Theorem 3.22). Let $c_{0}$ be the proalgebraic knot which is the infinite summation of (topological) knots given in Figure 3.5. Put

$$
\gamma_{0}:=\bigcirc-c_{0}+c_{0} \sharp c_{0}-c_{0} \sharp c_{0} \sharp c_{0}+c_{0} \sharp c_{0} \sharp c_{0} \sharp c_{0}-\cdots
$$

where $\bigcirc$ is the trivial knot and $\sharp$ is the product called the connected sum. Then Kontsevich (knot) invariant of $\gamma_{0}$ becomes trivial, i.e. $I\left(\gamma_{0}\right)=e$. Namely

$$
I^{-1}(e)=\gamma_{0} .
$$

We recall that the image $I(\circlearrowleft)$ of the unit $\bigcirc$, the trivial knot, under the Kontsevich isomorphism $I$ was calculated in BLT. Hence the above theorem could be regarded as a calculation in an opposite direction to their calculation.

The contents of the paper go as follows: $₫ 1$ is a review on Drinfeld's tools of Grothendieck-Teichmüller groups and their actions on braids, which serves for a good understanding of the actions of the groups on proalgebraic tangles given in \$2. Main results will be shown in 3 .

Convention. In this paper, $\mathbb{K}$ means a commutative field with characteristic 0 . (Actually we may more generally assume that it is a commutative ring containing the rational number field $\mathbb{Q}$.) The symbols $\mathbb{C}, \mathbb{R}, \mathbb{Q}_{p}, \mathbb{Z}_{p}, \mathbb{Z}$ and $\widehat{\mathbb{Z}}$ stand for the complex number field, the real number field, the $p$-adic number field ( $p$ : a prime), the $p$-adic integer ring, the integer ring and its profinite completion respectively.

\section{PRoAlgebraic BRAIDS AND INFINITESIMAL BRAIDS}

This is an expository section for non-experts on Drinfeld's works on the action of the proalgebraic Grothendieck-Teichmüller groups on proalgebraic braids and also on infinitesimal braids and also a short review on a relationship of the groups with the motivic Galois group.

1.1. The $G T$-action. We recall in Definition 1.2 explicitly the definition of the Grothendieck-Teichmüller group $G T(\mathbb{K})$, a proalgebraic group introduced by Drinfeld $\left[\mathrm{Dr}\right.$, and explain its action on the proalgebraic braids $\widehat{\mathbb{K}\left[B_{n}\right]}$ for $n \geqslant 2$ in Proposition 1.5.

Notation 1.1. (1) Let $B_{n}$ be the Artin braid group with $n$-strings $(n \geqslant 2)$ with standard generators $\sigma_{i}(1 \leqslant i \leqslant n-1)$ and defining relations $\sigma_{i} \sigma_{i+1} \sigma_{i}=\sigma_{i+1} \sigma_{i} \sigma_{i+1}$ and $\sigma_{i} \sigma_{j}=\sigma_{j} \sigma_{i}$ for $|i-j|>1$. The generator $\sigma_{i}$ in $B_{n}$ is depicted as in Figure 1.1. And for $b$ and $b^{\prime} \in B_{n}$, we draw the product $b \cdot b^{\prime} \in B_{n}$ as in Figure 1.2 (the order of product $b \cdot b^{\prime}$ is chosen to combine the bottom endpoints of $b$ with the top endpoints of $\left.b^{\prime}\right)$. 


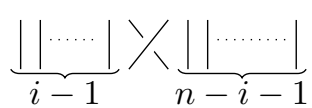

FiguRe 1.1. $\sigma_{i}$

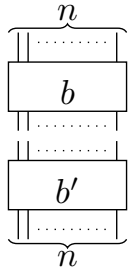

Figure $1.2 . \quad b \cdot b^{\prime}$

We denote the pure part of $B_{n}$ by $P_{n}$, i.e. the kernel of the natural homomorphism $P_{n} \rightarrow \mathfrak{S}_{n}$, and call it by the pure braid group. For $1 \leqslant i<j \leqslant n$, special elements

$$
x_{i, j}=x_{j, i}=\left(\sigma_{j-1} \cdots \sigma_{i+1}\right) \sigma_{i}^{2}\left(\sigma_{j-1} \cdots \sigma_{i+1}\right)^{-1}
$$

form a generating set of $P_{n}$. For $1 \leqslant a \leqslant a+\alpha<b \leqslant b+\beta \leqslant n$, we define

$$
\begin{aligned}
x_{a \cdots a+\alpha, b \cdots b+\beta}:= & \left(x_{a, b} x_{a, b+1} \cdots x_{a, b+\beta}\right) \cdot\left(x_{a+1, b} x_{a+1, b+1} \cdots x_{a+1, b+\beta}\right) \\
& \cdots\left(x_{a+\alpha, b} x_{a+\alpha, b+1} \cdots x_{a+\alpha, b+\beta}\right) \in P_{n} .
\end{aligned}
$$

They are drawn in Figure 1.3 and 1.4

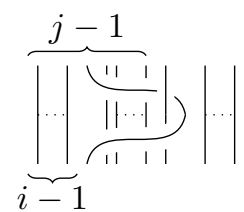

FiguRE 1.3. $x_{i j}$

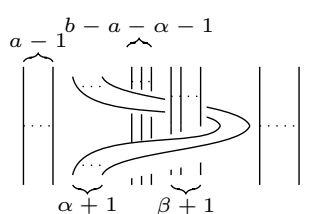

FigURE 1.4. $x_{a \cdots a+\alpha, b \cdots b+\beta}$

We mean $\widehat{\mathbb{K}\left[B_{n}\right]}$ by the completion of the group algebra $\mathbb{K}\left[B_{n}\right]$ with respect to the two-sided ideal $I$ generated by $\sigma_{i}-\sigma_{i}^{-1}$ for $1 \leqslant i \leqslant n-1$;

$$
\widehat{\mathbb{K}\left[B_{n}\right]}:={\underset{\varliminf}{N}}_{\lim } \mathbb{K}\left[B_{n}\right] / I^{N}
$$

(cf. [F6]). By abuse of notation, we denote the induced filtration on $\widehat{\mathbb{K}\left[B_{n}\right]}$ by the same symbol $\left\{I^{n}\right\}_{n \geqslant 0}$. It is checked that $\widehat{\mathbb{K}\left[B_{n}\right]}$ is a filtered Hopf algebra. We call its group-like part by the proalgebraic braid group and denote it by $B_{n}(\mathbb{K})$. It naturally admits a structure of a proalgebraic group over $\mathbb{K}$. We note that it is Hain's $[\mathrm{H}]$ relative completion of $B_{n}$ with respect to the natural projection $B_{n} \rightarrow \mathfrak{S}_{n}$.

(2) Similarly we denote its pure part by $\widehat{\mathbb{K}\left[P_{n}\right]}$. Namely

$$
\widehat{\mathbb{K}\left[P_{n}\right]}:=\underset{\lim _{N}}{\mathbb{K}}\left[P_{n}\right] / I_{0}^{N}
$$

with $I_{0}=I \cap \mathbb{K}\left[P_{n}\right]$. It is also a filtered Hopf algebra. The proalgebraic pure braid group $P_{n}(\mathbb{K})$ means its group-like part. We note that it is a unipotent (Malcev) completion of $P_{n}$ because $I_{0}$ forms an augmentation ideal of $\mathbb{K}\left[P_{n}\right]$.

(3) Let $F_{2}(\mathbb{K})$ be the prounipotent algebraic group over $\mathbb{K}$, the unipotent completion of the free group $F_{2}$ of rank 2 with two variables $x$ and $y$, that is, the group-like part of the Hopf algebra $\widehat{\mathbb{K}\left[F_{2}\right]}$ completed by the augmentation ideal. 
Similarly to the convention in [F6], for any $f \in F_{2}(\mathbb{K})$ and any homomorphism $\tau: F_{2}(\mathbb{K}) \rightarrow G(\mathbb{K})$ of proalgebraic groups sending $x \mapsto \alpha$ and $y \mapsto \beta$, the symbol $f(\alpha, \beta)$ stands for the image $\tau(f)$. Particularly for the (actually injective) homomorphism $F_{2}(\mathbb{K}) \rightarrow P_{n}(\mathbb{K})$ of proalgebraic groups sending $x \mapsto x_{a \cdots a+\alpha, b \cdots b+\beta}$ and $y \mapsto x_{b \cdots b+\beta, c \cdots c+\gamma}(1 \leqslant a \leqslant a+\alpha<b \leqslant b+\beta<c \leqslant c+\gamma \leqslant n)$, the image of $f \in F_{2}(\mathbb{K})$ is denoted by $f_{a \cdots a+\alpha, b \cdots b+\beta, c \cdots c+\gamma}$.

The proalgebraic group $G T(\mathbb{K})$ is defined by Drinfeld $[\mathrm{Dr}$ to be a subgroup of the automorphism (proalgebraic) group of $F_{2}(\mathbb{K})$.

Definition 1.2 ( $(\overline{\mathrm{Dr}}])$. The proalgebraic Grothendieck-Teichmüller group $G T(\mathbb{K})$ is the proalgebraic group over $\mathbb{K}$ whose set of $\mathbb{K}$-values points forms a subgroup of Aut $F_{2}(\mathbb{K})$ and is defined by

$G T(\mathbb{K}):=\left\{\begin{array}{l|l}\sigma \in \operatorname{Aut} F_{2}(\mathbb{K}) \mid \begin{array}{l}\sigma(x)=x^{\lambda}, \sigma(y)=f^{-1} y^{\lambda} f \text { for some }(\lambda, f) \in \mathbb{K}^{\times} \times F_{2}(\mathbb{K}) \\ \text { satisfying the three relations below. }\end{array}\end{array}\right\}$

$$
\begin{gathered}
f(x, y) f(y, x)=1 \quad \text { in } F_{2}(\mathbb{K}), \\
f(z, x) z^{m} f(y, z) y^{m} f(x, y) x^{m}=1 \text { in } F_{2}(\mathbb{K}) \text { with } z=(x y)^{-1}, m=\frac{\lambda-1}{2}, \\
f_{1,2,34} f_{12,3,4}=f_{2,3,4} f_{1,23,4} f_{1,2,3} \quad \text { in } P_{4}(\mathbb{K}) .
\end{gathered}
$$

The powers $x^{\lambda}, y^{\lambda}, x^{m}, y^{m}, z^{m}$ appearing in the above all make sense because $F_{2}(\mathbb{K})$ is the prounipotent completion of $F_{2}$. For $f_{1,2,34}$ etc., see Notation 1.1 .

We remark that each $\sigma \in G T(\mathbb{K})$ determines a pair $(\lambda, f)$ uniquely because the pentagon equation (1.3) implies that $f$ belongs to the commutator of $F_{2}(\mathbb{K})$. By abuse of notation, we occasionally express the pair $(\lambda, f)$ to represents $\sigma$ and denote as $\sigma=(\lambda, f) \in G T(\mathbb{K})$.

The above set-theoretically defined $G T(\mathbb{K})$ forms indeed a proalgebraic group whose product is induced from that of $\operatorname{Aut}_{2}(\mathbb{K})$ and is given by 1

$$
\left(\lambda_{2}, f_{2}\right) \circ\left(\lambda_{1}, f_{1}\right):=\left(\lambda_{2} \lambda_{1}, f_{1}\left(f_{2} x^{\lambda_{2}} f_{2}^{-1}, y^{\lambda_{2}}\right) \cdot f_{2}\right)=\left(\lambda_{2} \lambda_{1}, f_{2} \cdot f_{1}\left(x^{\lambda_{2}}, f_{2}^{-1} y^{\lambda_{2}} f_{2}\right)\right) .
$$

The first equality is the definition and the second equality can be easily verified. We denote the subgroup of $G T(\mathbb{K})$ with $\lambda=1$ by $G T_{1}(\mathbb{K})$;

$$
G T_{1}(\mathbb{K}):=\{\sigma=(\lambda, f) \in G T(\mathbb{K}) \mid \lambda=1\} .
$$

It is easily seen that it forms a proalgebraic unipotent subgroup of $G T(\mathbb{K})$.

Remark 1.3. In some literatures, (1.1), (1.2) and (1.3) are called 2-cycle, 3-cycle and 5-cycle relation respectively. The author often calls (1.1) and (1.2) by two hexagon equations and (1.3) by one pentagon equation because they reflect the three axioms, two hexagon and one pentagon axioms, of braided monoidal (tensor) categories [JS. We remind that (1.3) represents

$$
f\left(x_{12}, x_{23} x_{24}\right) f\left(x_{13} x_{23}, x_{34}\right)=f\left(x_{23}, x_{34}\right) f\left(x_{12} x_{13}, x_{24} x_{34}\right) f\left(x_{12}, x_{23}\right) \quad \text { in } P_{4}(\mathbb{K}) .
$$

\footnotetext{
${ }^{1}$ For our purpose to make 1.5 not anti-homomorphic but homomorphic, we reverse the order of the product given in the original paper $\mathrm{Dr}$.
} 
In several literatures such as [I, F3, the equation (1.3) is replaced by a different (more symmetric) formulation:

$$
f\left(x_{12}^{*}, x_{23}^{*}\right) f\left(x_{34}^{*}, x_{45}^{*}\right) f\left(x_{51}^{*}, x_{12}^{*}\right) f\left(x_{23}^{*}, x_{34}^{*}\right) f\left(x_{45}^{*}, x_{51}^{*}\right)=1 \quad \text { in } P_{5}^{*}(\mathbb{K})
$$

where $P_{5}^{*}$ is the pure sphere braid group with 5-strings.

The author actually showed that the pentagon equation implies two hexagon equations:

Proposition $1.4([\mathrm{F5}])$. Let $\mathbb{K}$ be an algebraically closed field of characteristic 0 . For each $f \in F_{2}(\mathbb{K})$ satisfying (1.3), there always exists (actually unique up to signature) $\lambda \in \mathbb{K}$ such that the pair $(\lambda, f)$ satisfies the two hexagon equations (1.1) and (1.2).

The following Drinfeld's $G T(\mathbb{K})$-action on $\widehat{\mathbb{K}\left[B_{n}\right]}$ plays a fundamental role in our paper here.

Proposition $1.5(\overline{\mathrm{Dr}})$. Let $n \geqslant 2$. There is a continuous $G T(\mathbb{K})$-action on the filtered Hopf algebra $\widehat{\mathbb{K}\left[B_{n}\right]}$

$$
\rho_{n}: G T(\mathbb{K}) \rightarrow \text { Aut } \widehat{\mathbb{K}\left[B_{n}\right]}
$$

which is induced by, for each $\sigma=(\lambda, f) \in G T(\mathbb{K})$,

$$
\rho_{n}(\sigma):\left\{\begin{array}{llll}
\sigma_{1} & \mapsto & & \sigma_{1}^{\lambda}, \\
\sigma_{i} & \mapsto & f_{1 \cdots i-1, i, i+1}^{-1} & \sigma_{i}^{\lambda} f_{1 \cdots i-1, i, i+1}
\end{array} \quad(2 \leqslant i \leqslant n-1) .\right.
$$

Here $\sigma_{i}^{\lambda}:=\sigma_{i} \cdot\left(\sigma_{i}^{2}\right)^{\frac{\lambda-1}{2}} \in \widehat{\mathbb{K}\left[B_{n}\right]}$ and $f_{1 \cdots i-1, i, i+1}=f\left(x_{1 i} x_{2 i} \cdots x_{i-1, i}, x_{i, i+1}\right) \in$ $P_{n}(\mathbb{K})$ (see Notation 1.11). .It is well defined because $\sigma_{i}^{2}$ belongs to the unipotent completion $P_{n}(\mathbb{K})$ and $\frac{\lambda-1}{2}$-th power of $\sigma_{i}^{2}$ makes sense in $P_{n}(\mathbb{K})$. We denote $\rho_{n}(\sigma)(b)$ simply by $\sigma(b)$ when there is no confusion.

We note that $\rho_{n}$ is injective when $n \geqslant 3$.

Remark 1.6. For each prime $l$, there are natural homomorphisms $\widehat{B}_{n} \rightarrow B_{n}\left(\mathbb{Q}_{l}\right)$ and $\widehat{P}_{n} \rightarrow P_{n}\left(\mathbb{Q}_{l}\right)$. Hence we have

$$
\widehat{B}_{n} \rightarrow \widehat{\mathbb{Q}_{l}\left[B_{n}\right]} .
$$

By natural homomorphisms $\widehat{\mathbb{Z}} \rightarrow \mathbb{Q}_{l}, \widehat{F}_{2} \rightarrow F_{2}\left(\mathbb{Q}_{l}\right)$ and $\widehat{P}_{4} \rightarrow P_{4}\left(\mathbb{Q}_{l}\right)$, we obtain a continuous group homomorphism

$$
\widehat{G T} \rightarrow G T\left(\mathbb{Q}_{l}\right)
$$

from the profinite Grothendieck-Teichmüller group $\widehat{G T}$ (cf. [F6]). By direct calculations, it can be verified that the above two homomorphisms are consistent with the $G T\left(\mathbb{Q}_{l}\right)$-action on $\widehat{\mathbb{Q}_{l}\left[B_{n}\right]}$ in Proposition $\left[1.5\right.$ and the $\widehat{G T}$-action on $\widehat{B}_{n}$ (given in $[\mathrm{Dr}$ ] and see also [F6]).

In $₫ 3$, we will extend the action on proalgebraic braids to the one on proalgebraic tangles and will show that actually the above action on proalgebraic pure braid groups is realized as an inner automorphism of proalgebraic string links (Corollary 3.15). 
1.2. The GRT-action. We recall explicitly Drinfeld's definitions of the graded Grothendieck-Teichmüller group $G R T(\mathbb{K})$ in Definition 1.9. We discuss their actions on the algebra ${\widehat{U \mathfrak{b}_{n}}}_{n}$ of infinitesimal braids for $n \geqslant 2$ in Proposition 1.12

Notation 1.7. (1) Let $\mathfrak{p}_{n}$ be the infinitesimal pure braid Lie algebra with $n$-strings $(n \geqslant 2)$ with standard generators $t_{i j}(1 \leqslant i, j \leqslant n)$ and defining relations $t_{i i}=0$, $t_{i j}=t_{j i},\left[t_{i j}, t_{i k}+t_{j k}\right]=0$ and $\left[t_{i j}, t_{k l}\right]=0$ when $i, j, k, l$ all differ. For $1 \leqslant a \leqslant$ $a+\alpha<b \leqslant b+\beta \leqslant n$, we define

$$
t_{a \cdots a+\alpha, b \cdots b+\beta}:=\sum_{0 \leqslant i \leqslant \alpha, 0 \leqslant j \leqslant \beta} t_{a+i, b+j} \in \mathfrak{p}_{n} .
$$

We denote by $U \mathfrak{p}_{n}$ its enveloping algebra and by $\widehat{U \mathfrak{p}_{n}}$ its completion with respect to its augmentation ideal.

(2) We denote $\mathfrak{S}_{n}$ to be the symmetric group acting on $\{1,2, \ldots, n\}(n \geqslant 1)$ and $\tau_{i, i+1}$ in $\mathfrak{S}_{n}$ to be the transpose of $i$ and $i+1(1 \leqslant i \leqslant n-1)$. The group $\mathfrak{S}_{n}$ acts $\widehat{U \mathfrak{p}_{n}}$ by $\tau \cdot t_{i j}=t_{\tau^{-1}(i), \tau^{-1}(j)}$ for $\tau \in \mathfrak{S}_{n}$ and $1 \leqslant i, j \leqslant n$. We may consider the crossed product (cf. [M] etc.)

$$
\widehat{U \mathfrak{b}_{n}}:=\mathbb{K}\left[\mathfrak{S}_{n}\right] *{\widehat{U \mathfrak{p}_{n}}}
$$

It is $\mathbb{K}\left[\mathfrak{S}_{n}\right] \otimes_{\mathbb{K}} \widehat{U \mathfrak{p}_{n}}$ as vector space with the product structure given by

$$
\left(\tau_{1} \otimes t_{i_{1} j_{1}}\right) \cdot\left(\tau_{2} \otimes t_{i_{2} j_{2}}\right):=\tau_{1} \tau_{2} \otimes\left(t_{\tau_{2}^{-1}\left(i_{1}\right) \tau_{2}^{-1}\left(j_{1}\right)} \cdot t_{i_{2} j_{2}}\right)
$$

for $\tau_{1}, \tau_{2} \in \mathfrak{S}_{n}$. By abuse of notation, occasionally $\tau$ indicate $\tau \otimes 1$ for $\tau \in \mathbb{K}\left[\mathfrak{S}_{n}\right]$ and $t$ indicate $1 \otimes t$ for $t \in \widehat{U_{\mathfrak{p}}}$ in this paper. Hence we have

$$
\tau \cdot t_{i j}=t_{\tau(i) \tau(j)} \cdot \tau \quad\left(=\tau \otimes t_{i j}\right)
$$

for $\tau \in \mathfrak{S}_{n}$. We occasionally depict $t_{i j} \in \widehat{U \mathfrak{p}_{n}}$ as the diagram with $n$ vertical lines and a dotted horizontal line (called a chord) connecting $i$-th and $j$-th lines and $\tau \in \mathfrak{S}_{n}$ as the diagram connecting each $i$-th point on the bottom with $\tau(i)$-th point on the top by an interval. The order of the product $b \cdot b^{\prime}$ is chosen to combine the bottom endpoints of $b$ with the top endpoints of $b^{\prime}$. By putting $\operatorname{deg} t_{i j}=1(1 \leqslant$ $i, j \leqslant n)$ and $\operatorname{deg} \tau=0\left(\tau \in \mathfrak{S}_{n}\right)$, we can show that both $\widehat{U \mathfrak{p}_{n}}$ and $\widehat{U \mathfrak{b}_{n}}$ carry structures of graded Hopf algebras.

(3) Let $\mathfrak{f}_{2}$ be the free Lie algebra over $\mathbb{K}$ with two variables $A$ and $B$ and $\widehat{U \mathfrak{f f}_{2}}$ be its completed Hopf algebra. Again similarly, for any $g \in \widehat{U \mathfrak{f f}_{2}}$ and any algebra homomorphism $\tau: \widehat{U_{f_{2}}} \rightarrow S$ sending $A \mapsto v$ and $B \mapsto w$, the symbol $g(v, w)$ stands for the image $\tau(g)$. Particularly for the (actually injective) homomorphism $\widehat{U_{\mathfrak{F}_{2}}} \rightarrow \widehat{U \mathfrak{p p}_{n}}$ sending $A \mapsto t_{a \cdots a+\alpha, b \cdots b+\beta}$ and $B \mapsto t_{b \cdots b+\beta, c \cdots c+\gamma}(1 \leqslant a \leqslant a+\alpha<$ $b \leqslant b+\beta<c \leqslant c+\gamma \leqslant n)$, the image of $g \in \widehat{U \mathfrak{U f}_{2}}$ is denoted by $g_{a \cdots a+\alpha, b \cdots b+\beta, c \cdots c+\gamma} \in$ $\widehat{U \mathfrak{p}_{n}}$.

We note that $\widehat{U \mathfrak{p}_{n}}$ is not algebraically generated by $t_{i, i+1}(1 \leqslant i \leqslant n-1)$ while the following holds for $\widehat{U \mathfrak{b}_{n}}$.

Lemma 1.8. The algebra ${\widehat{U \mathfrak{b}_{n}}}_{\text {is }}$ algebraically generated by $t_{i, i+1}$ and $\tau_{i, i+1}$ for $1 \leqslant i \leqslant n-1$.

Proof. The elements $\tau_{i, i+1}$ generate $\mathfrak{S}_{n}$ and, by (1.7), any $t_{k l}$ is obtained from $t_{i, i+1}$ and $\tau_{i, i+1}(1 \leqslant i \leqslant n-1)$, which yields our claim. 
The proalgebraic group $G R T_{1}(\mathbb{K})$ is defined by Drinfeld $\underline{\mathrm{Dr}}$ to be a proalgebraic subgroup of the automorphism (proalgebraic) group of $\exp \mathfrak{f}_{2}$.

Definition $1.9(\underline{\mathrm{Dr}})$. The proalgebraic graded Grothendieck-Teichmüller group $G R T(\mathbb{K})$ is the subgroup of Aut $\exp \mathfrak{f}_{2}$ defined by

$G R T(\mathbb{K}):=\left\{\begin{array}{l|l}\sigma \in \text { Aut exp } \mathfrak{f}_{2} \mid \begin{array}{l}\sigma\left(e^{A}\right)=e^{A / c}, \sigma\left(e^{B}\right)=g^{-1} e^{B / c} g \text { for some } c \in \mathbb{K}^{\times} \\ \text {and } g \in \exp \mathfrak{f}_{2} \text { satisfying two hexagon equations } \\ \text { (1.8)-(1.9) and one pentagon equation (1.10) below. }\end{array}\end{array}\right\}$

$$
\begin{gathered}
g(A, B) g(B, A)=1 \quad \text { in } \exp \mathfrak{f}_{2}, \\
g(C, A) g(B, C) g(A, B)=1 \quad \text { in } \exp \mathfrak{f}_{2} \text { with } C=-A-B, \\
g_{1,2,34} g_{12,3,4}=g_{2,3,4} g_{1,23,4} g_{1,2,3} \quad \text { in } \exp \mathfrak{p}_{4} .
\end{gathered}
$$

Similarly to Definition 1.2, we remark that each $\sigma \in G R T(\mathbb{K})$ determines a pair $(c, g)$ uniquely. By abuse of notation, we occasionally express the pair $(c, g)$ to represents $\sigma \in G R T(\mathbb{K})$ and denote as $\sigma=(c, g) \in G R T(\mathbb{K})$.

The above set-theoretically defined $G R T(\mathbb{K})$ forms indeed a proalgebraic group whose product is induced from that of Aut $\exp \mathfrak{f}_{2}(\mathbb{K})$ and is given by

$$
\left(c_{2}, g_{2}\right) \circ\left(c_{1}, g_{1}\right)=\left(c_{2} c_{1}, g_{1}\left(g_{2} \frac{A}{c_{2}} g_{2}^{-1}, \frac{B}{c_{2}}\right) \cdot g_{2}\right)=\left(c_{2} c_{1}, g_{2} \cdot g_{1}\left(\frac{A}{c_{2}}, g_{2}^{-1} \frac{B}{c_{2}} g_{2}\right)\right) \text {. }
$$

The first equality is the definition and the second equality can be easily verified. Notice the simple equality $(1, g) \circ(c, 1)=(c, g)$. We denote the subgroup of $G R T(\mathbb{K})$ with $c=1$ by $G R T(\mathbb{K})_{1}$, i.e. $G R T_{1}(\mathbb{K}):=\{\sigma=(c, g) \in G R T(\mathbb{K}) \mid c=1\}$. It is easily seen that it forms a proalgebraic unipotent subgroup of $G R T(\mathbb{K})$.

Remark 1.10. The symbol GRT stands for 'graded Grothendieck-Teichmüller group.' Indeed its grading on $G R T_{1}(\mathbb{K})$ is equipped by the action of $\mathbb{G}_{m}(\mathbb{K})\left(=\mathbb{K}^{\times}\right)$ given by

$$
(1, g(A, B)) \mapsto\left(1, g\left(\frac{A}{c}, \frac{B}{c}\right)\right)
$$

for $c \in \mathbb{K}^{\times}$and $g \in G R T_{1}(\mathbb{K})$, which is reformulated by $(1, g) \mapsto(c, 1) \circ(1, g)$. Thus we have, by the action,

$$
G R T(\mathbb{K})=\mathbb{K}^{\times} \ltimes G R T_{1}(\mathbb{K}) .
$$

Two specific elements of $G R T(\mathbb{K})$ are known.

Example 1.11. (1) The $p$-adic Drinfeld associator $\Phi_{\mathrm{KZ}}^{p}(A, B)$, a $p$-adic analogue of the Drinfeld (KZ-)associator (cf. Example 1.16) is a non-commutative formal power series whose coefficients are $p$-adic multiple zeta values [F2]. It was constructed as a regularized holonomy of the $p$-adic KZ-equation and was shown in [F4] by the results of $[\mathrm{U}]$ that it belongs to $G R T_{1}(\mathbb{K})$ with $\mathbb{K}=\mathbb{Q}_{p}$.

(2) The $p$-adic Deligne associator $\Phi_{\mathrm{De}}^{p}(A, B)$, a variant of the above $\Phi_{\mathrm{KZ}}^{p}(A, B)$ (cf. [F4]) is shown in [U] to be in $G R T_{1}(\mathbb{K})$ with $\mathbb{K}=\mathbb{Q}_{p}$. It was in [F4] shown that each of its coefficients is given by a certain polynomial combination of $p$-adic multiple zeta values.

\footnotetext{
${ }^{2}$ We remark again that, for our purpose, we reverse the order of the product given in $[\mathrm{Dr}]$.
} 
The following $G R T(\mathbb{K})$-action on $\widehat{U \mathfrak{b}_{n}}$ was explicitly presented neither in Drinfeld's paper Dr] nor Bar-Natan's paper [Ba3], where they showed $G R T_{1}(\mathbb{K})$-action there.

Proposition 1.12. Let $n \geqslant 2$. There is a continuous $G R T(\mathbb{K})$-action on the graded Hopf algebra $\widehat{U \mathfrak{b}_{n}}$

$$
\rho_{n}: G R T(\mathbb{K}) \rightarrow \text { Aut } \widehat{U \mathfrak{b}_{n}}
$$

which is induced by, for each $\sigma=(c, g) \in G R T(\mathbb{K})$,

$$
\rho_{n}(\sigma):\left\{\begin{array}{lllll}
t_{1,2} & \mapsto & & \frac{t_{1,2}}{c}, & \\
t_{i, i+1} & \mapsto & g_{1 \cdots i-1, i, i+1}^{-1} & \frac{t_{i, i+1}}{c} g_{1 \cdots i-1, i, i+1} & (2 \leqslant i \leqslant n-1), \\
\tau_{1,2} & \mapsto & & \tau_{1,2}, & \\
\tau_{i, i+1} & \mapsto & g_{1 \cdots i-1, i, i+1}^{-1} & \tau_{i, i+1} g_{1 \cdots i-1, i, i+1} & (2 \leqslant i \leqslant n-1) .
\end{array}\right.
$$

We recall that $\tau_{i, i+1}$ means the transpose of $i$ and $i+1$ in $\mathfrak{S}_{n}$. We again note that $\rho_{n}$ is injective when $n \geqslant 3$.

In $\$ 3$ we will extend the above action on infinitesimal braids to the one on chord diagrams and will show that actually the above action on infinitesimal braids is realized as an inner automorphism of chord diagrams.

The associated Lie algebra $\mathfrak{g r t}_{1}$ of $G R T_{1}$, which was independently introduced by Ihara [I] and called the stable derivation algebra, is equipped grading by the $\mathbb{G}_{m}$-action (1.12).

Conjecture $1.13([\mathrm{De}, \overline{\mathrm{Dr}}, \mathrm{I}])$. The graded Lie algebra $\mathfrak{g r t}_{1}$ is freely generated by one element in each degree $3,5,7, \ldots$.

Remark 1.14. By Br1], we know that $\mathfrak{g r t}_{1}$ contains such a free Lie subalgebra (see Remark 1.23 below).

1.3. Associators. We recall Drinfeld's definition of the associator set $M(\mathbb{K})$ in Definition 1.15 and its $(G R T(\mathbb{K}), G T(\mathbb{K}))$-bitorsor structure in Proposition 1.18. Then we will explain how associators give isomorphisms between $\widehat{\mathbb{K}\left[B_{n}\right]}$ and $\widehat{U \mathfrak{b}_{n}}$ in Proposition 1.19 .

Definition $1.15(\underline{\mathrm{Dr}})$. The associator set $M(\mathbb{K})$ is the proalgebraic variety whose set of $\mathbb{K}$-valued points is given by

$M(\mathbb{K}):=\left\{p=(\mu, \varphi) \in \mathbb{K} \times \exp \mathfrak{f}_{2} \mid \mu \in \mathbb{K}^{\times}\right.$and $(\mu, \varphi)$ satisfies (1.8), (1.10) and (1.14). $\}$

$$
\exp \left\{\frac{\mu A}{2}\right\} \varphi(C, A) \exp \left\{\frac{\mu C}{2}\right\} \varphi(B, C) \exp \left\{\frac{\mu B}{2}\right\} \varphi(A, B)=1 \quad \text { in } \exp \mathfrak{f}_{2}
$$

with $C=-A-B$. For each fixed $\mu_{0} \in \mathbb{K}$, define the proalgebraic variety $M_{\mu_{0}}(\mathbb{K})$ by

$$
M_{\mu_{0}}(\mathbb{K}):=\left\{\varphi \in \exp _{2} \mid \varphi \text { satisfies (1.8), (1.10) and (1.14) with } \mu=\mu_{0}\right\} .
$$

Hence we have $M_{0}(\mathbb{K})=G R T_{1}(\mathbb{K})$. Three examples of associators are known:

Example 1.16. (1) The KZ-associator, also known as the Drinfeld associator, $\Phi_{\mathrm{KZ}}(A, B)$ is a non-commutative formal power series whose coefficients are multiple zeta values. It was constructed by Drinfeld $[\mathrm{Dr}$ ] as a regularized holonomy of the 
KZ-equation and was shown by him that it belongs to $M_{\mu}(\mathbb{K})$ with $\mathbb{K}=\mathbb{C}$ and $\mu= \pm 2 \pi \sqrt{-1}$. It is known to be expressed as follows:

$$
\begin{gathered}
\Phi_{\mathrm{KZ}}(A, B)=1+\sum_{\substack{m, k_{1}, \ldots, k_{m} \in \mathbb{N} \\
k_{m}>1}}(-1)^{m} \zeta\left(k_{1}, \cdots, k_{m}\right) A^{k_{m}-1} B \cdots A^{k_{1}-1} B \\
+ \text { (regularized terms). }
\end{gathered}
$$

Here $\zeta\left(k_{1}, \cdots, k_{m}\right)$ is the multiple zeta value (MZV in short), the real number defined by the following power series

$$
\zeta\left(k_{1}, \cdots, k_{m}\right):=\sum_{0<n_{1}<\cdots<n_{m}} \frac{1}{n_{1}^{k_{1}} \cdots n_{m}^{k_{m}}}
$$

for $m, k_{1}, \ldots, k_{m} \in \mathbb{N}\left(=\mathbb{Z}_{>0}\right)$ with $k_{m}>1$ (its convergent condition). All of the coefficients of $\Phi_{\mathrm{KZ}}$ (including its regularized terms) are explicitly calculated in terms of MZV's in F1 Proposition 3.2.3 by Le-Murakami's method in LM2.

(2) The Deligne associator $\Phi_{\mathrm{De}}(A, B)([\mathrm{Br} 2])$ (denoted by $\Phi_{\mathrm{KZ}}^{-}(A, B)$ in [F4]) is a non-commutative formal power series in $M_{\mu}(\mathbb{K})$ with $\mathbb{K}=\mathbb{R}$ and $\mu=1$ which is located in the 'middle' of $\Phi_{\mathrm{KZ}}\left(\frac{1}{2 \pi \sqrt{-1}} A, \frac{1}{2 \pi \sqrt{-1}} B\right)$ and $\Phi_{\mathrm{KZ}}\left(\frac{-1}{2 \pi \sqrt{-1}} A, \frac{-1}{2 \pi \sqrt{-1}} B\right)$. Its explicit relationship with the above $\Phi_{\mathrm{KZ}}(A, B)$ is given in [F4] Lemma 2.25.

(3) The AT-associator $\Phi_{\mathrm{AT}}(A, B)$ is another associator. It was constructed by Alekseev and Torossian AT1 as a holonomy of AT-connection, a certain nonholomorphic flat connection on a certain configuration space. Ševera and Willwacher [SW] showed that it belongs to $M_{\mu}(\mathbb{K})$ with $\mathbb{K}=\mathbb{R}$ and $\mu=1$. Rossi and Willwacher showed that $\Phi_{\mathrm{AT}} \neq \Phi_{\mathrm{De}}$ in $[\mathrm{RW}$.

We remark again that the author also in this setting showed that the pentagon equation implies two hexagon equations.

Proposition 1.17 ([F6]). Let $\mathbb{K}$ be an algebraically closed field of characteristic 0 . For each $\varphi \in \exp \mathfrak{f}_{2}$ satisfying (1.10), there always exists (actually unique up to signature) $\mu \in \mathbb{K}$ such that the pair $p=(\mu, \varphi)$ satisfies the two hexagon equations (1.8) and (1.14).

It was shown by Drinfeld that $G R T(\mathbb{K})$ acts freely and transitively on $M(\mathbb{K})$ from the left, $G T(\mathbb{K})$ acts freely and transitively on $M(\mathbb{K})$ from the right, and these two actions are commutative:

Proposition $1.18([\mathrm{Dr})$. The associator set $M(\mathbb{K})$ forms a $(G R T(\mathbb{K}), G T(\mathbb{K}))$ bitorsor by the left $G R T(\mathbb{K})$-action given by

$$
(c, g) \circ(\mu, \varphi):=\left(\frac{\mu}{c}, \varphi\left(g \frac{A}{c} g^{-1}, \frac{B}{c}\right) \cdot g\right)=\left(\frac{\mu}{c}, g \cdot \varphi\left(\frac{A}{c}, g^{-1} \frac{B}{c} g\right)\right)
$$

for $(c, g) \in G R T(\mathbb{K})$ and $(\mu, \varphi) \in M(\mathbb{K})$ and the right $G T(\mathbb{K})$-action given by

$$
(\mu, \varphi) \circ(\lambda, f):=\left(\lambda \mu, f\left(\varphi e^{\mu A} \varphi^{-1}, e^{\mu B}\right) \cdot \varphi\right)=\left(\lambda \mu, \varphi \cdot f\left(e^{\mu A}, \varphi^{-1} e^{\mu B} \varphi\right)\right)
$$

for $(\mu, \varphi) \in M(\mathbb{K})$ and $(\lambda, f) \in G T(\mathbb{K})$.

We must note again that we reverse the order of the product given in the paper $\mathrm{Dr}$ for our purpose. Drinfeld $\mathrm{Dr}$ showed that associators give an isomorphism between $\widehat{\mathbb{K}\left[B_{n}\right]}$ and $\widehat{U \mathfrak{b}_{n}}$. 
Proposition $1.19(\overline{\mathrm{Dr}})$. Let $n \geqslant 2$. The $(G R T(\mathbb{K}), G T(\mathbb{K}))$-bitorsor $M(\mathbb{K})$ is mapped to the $\left(\operatorname{Aut}\left(\widehat{U \mathfrak{U b}_{n}}\right)\right.$, Aut $\left.\left(\widehat{\mathbb{K}\left[B_{n}\right]}\right)\right)$-bitorsor $\operatorname{Isom}\left(\widehat{\mathbb{K}\left[B_{n}\right]}, \widehat{U \mathfrak{U b}_{n}}\right)$ by the map

$$
\rho_{n}: M(\mathbb{K}) \rightarrow \operatorname{Isom}\left(\widehat{\mathbb{K}\left[B_{n}\right]}, \widehat{U_{\mathfrak{b}}}\right)
$$

induced by, for each $p=(\mu, \varphi)$,

$\rho_{n}(p):\left\{\begin{array}{llll}\sigma_{1} & \mapsto & & \tau_{1,2} \cdot \exp \left\{\frac{\mu t_{12}}{2}\right\}, \\ \sigma_{i} & \mapsto \quad \varphi_{1 \cdots i-1, i, i+1}^{-1} \cdot & \tau_{i, i+1} \cdot \exp \left\{\frac{\mu t_{i, i+1}}{2}\right\} \cdot \varphi_{1 \cdots i-1, i, i+1}(2 \leqslant i \leqslant n-1) .\end{array}\right.$

It is a morphism as bitorsors, i.e. it is compatible with (1.5) and (1.13).

We again note that $\rho_{n}$ is injective when $n \geqslant 3$.

1.4. The motivic Galois group. We briefly review the formulations of the motivic Galois groups and their torsor(consult also $\mathrm{A}$ as a nice exposition). We also review their relationship with the torsor of the Grothendieck-Teichmüller groups discussed in our previous subsections.

The triangulated category $D M(\mathbb{Q})_{\mathbb{Q}}$ of mixed motives over $\mathbb{Q}$ (a part of an idea of mixed motives is explained in De $\S 1$ ) was constructed by Hanamura, Levine and Voevodsky. Tate motives $\mathbb{Q}(n)(n \in \mathbb{Z})$ are (Tate) objects of the category. Let $D M T(\mathbb{Q})_{\mathbb{Q}}$ be the triangulated sub-category of $D M(\mathbb{Q})_{\mathbb{Q}}$ generated by Tate motives $\mathbb{Q}(n)(n \in \mathbb{Z})$. By the work of Levine a neutral tannakian $\mathbb{Q}$-category $M T(\mathbb{Q})=M T(\mathbb{Q})_{\mathbb{Q}}$ of mixed Tate motives over $\mathbb{Q}$ is extracted by taking the heart with respect to a $t$-structure of $D M T(\mathbb{Q})_{\mathbb{Q}}$. Deligne and Goncharov DeG] introduced the full subcategory $M T(\mathbb{Z})=M T(\mathbb{Z})_{\mathbb{Q}}$ of mixed Tate motives over Spec $\mathbb{Z}$ inside of $M T(\mathbb{Q})_{\mathbb{Q}}$. The category $M T(\mathbb{Z})$ forms a neutral tannakian $\mathbb{Q}$-category and association of each object $M \in M T(\mathbb{Z})$ with the underlying $\mathbb{Q}$-linear space of its Betti and de Rham realizations give the fiber functor $\omega_{\mathrm{Be}}$ and $\omega_{\mathrm{DR}}$ respectively.

Definition 1.20. For $* \in\{\mathrm{Be}, \mathrm{DR}\}$, the motivic Galois group $\mathrm{Gal}_{*}^{\mathcal{M}}(\mathbb{Z})$ is defined to be the corresponding tannakian fundamental group of $M T(\mathbb{Z})$, that is, the pro$\mathbb{Q}$-algebraic group defined by $\underline{\operatorname{Aut}}^{\otimes}\left(M T(\mathbb{Z}): \omega_{*}\right)$.

For $*, *^{\prime} \in\{\mathrm{Be}, \mathrm{DR}\}$, we denote the corresponding tannakian fundamental torsor $\underline{\operatorname{Isom}}^{\otimes}\left(M T(\mathbb{Z}): \omega_{*}, \omega_{*^{\prime}}\right)$ by $\mathrm{Gal}_{*, *^{\prime}}^{\mathcal{M}}(\mathbb{Z})$. This is a $\left(\mathrm{Gal}_{*^{\prime}}^{\mathcal{M}}(\mathbb{Z}), \mathrm{Gal}_{*}^{\mathcal{M}}(\mathbb{Z})\right)$-bitorsor. We note that $\mathrm{Gal}_{*, *}^{\mathcal{M}}(\mathbb{Z})=\operatorname{Gal}_{*}^{\mathcal{M}}(\mathbb{Z})$. By the fundamental theorem of tannakian category theory, each fiber functor $\omega_{*}$ induces an equivalence of categories

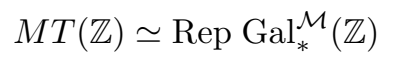

where the right hand side stands for the category of finite dimensional $\mathbb{Q}$-vector spaces equipped with $\mathrm{Gal}_{*}^{\mathcal{M}}(\mathbb{Z})$-action.

Remark 1.21. For $*, *^{\prime} \in\{\mathrm{Be}, \mathrm{DR}\}$, the action of $\mathrm{Gal}_{*}^{\mathcal{M}}(\mathbb{Z})$ on $\omega_{*}(\mathbb{Q}(1)) \simeq \mathbb{Q}$ defines a surjection $\mathrm{Gal}_{*}^{\mathcal{M}}(\mathbb{Z}) \rightarrow \mathbb{G}_{m}$ and its kernel $\mathrm{Gal}_{*}^{\mathcal{M}}(\mathbb{Z})_{1}$ is the unipotent radical of $\mathrm{Gal}_{*}^{\mathcal{M}}(\mathbb{Z})$. For $*=\mathrm{DR}$, there is a natural splitting $\tau: \mathbf{G}_{m} \rightarrow \mathrm{Gal}_{\mathrm{DR}}^{\mathcal{M}}(\mathbb{Z})$ which gives a negative grading on its associated Lie algebra $\operatorname{LieGal}_{\mathrm{DR}}^{\mathcal{M}}(\mathbb{Z})_{1}$. By the axiom on the structure of the category $M T(\mathbb{Z})$, it is known that the Lie algebra is the graded Lie algebra freely generated by one element in each degree $-3,-5,-7, \ldots$. (consult [De $\S 8$ for the full story). 
The motivic fundamental group $\pi_{1}^{\mathcal{M}}\left(\mathbb{P}^{1} \backslash\{0,1, \infty\}: \overrightarrow{01}\right)$ constructed in [DeG] $\S 4$ is a (pro-)object of $M T(\mathbb{Z})$. The KZ-associator (cf. Example 1.16) is essential in describing the Hodge realization of the motive (cf. [A, DeG, F4]). Since its Betti and de Rham realization is given by $F_{2}(\mathbb{Q})$ and $\exp \mathfrak{f}_{2}$, the motivic Galois groups, $\operatorname{Gal}_{\mathrm{Be}}^{\mathcal{M}}(\mathbb{Z})$ and $\mathrm{Gal}_{\mathrm{DR}}^{\mathcal{M}}(\mathbb{Z})$, acts there respectively. The tannakian equivalence (1.17) induces a morphism of bitorsors

$$
\Psi: \mathrm{Gal}_{\mathrm{Be}, \mathrm{DR}}^{\mathcal{M}}(\mathbb{Z}) \rightarrow \operatorname{Isom}\left(F_{2}(\mathbb{Q}), \exp \mathfrak{f}_{2}\right)
$$

from the $\left(\operatorname{Gal}_{\mathrm{DR}}^{\mathcal{M}}(\mathbb{Z}), \mathrm{Gal}_{\mathrm{Be}}^{\mathcal{M}}(\mathbb{Z})\right)$-bitorsor $\operatorname{Gal}_{\mathrm{Be}, \mathrm{DR}}^{\mathcal{M}}(\mathbb{Z})$ to the (Aut exp $\mathfrak{f}_{2}, \mathrm{Aut}_{2}(\mathbb{Q})$ )bitorsor $\operatorname{Isom}\left(F_{2}(\mathbb{Q}), \exp \mathfrak{f}_{2}\right)$. The following has been conjectured (Deligne-Ihara conjecture) and finally proved by Brown by using Zagier's relation on MZV's.

Theorem 1.22 ([Br1] $)$. The map $\Psi$ is injective.

It is a proalgebraic group analogue of the so-called Belyı's theorem $[\mathrm{Be}$ in the profinite group setting. The theorem says that all unramified mixed Tate motives over Spec $\mathbb{Z}$ are associated with MZV's.

Remark 1.23. We recall that our $(G R T(\mathbb{Q}), G T(\mathbb{Q}))$-bitorsor $M(\mathbb{Q})$ is naturally injected to the $\left(\operatorname{Aut} \exp \mathfrak{f}_{2}, \operatorname{Aut} F_{2}(\mathbb{Q})\right)$-bitorsor $\operatorname{Isom}\left(F_{2}(\mathbb{Q})\right.$, $\left.\exp \mathfrak{f}_{2}\right)$ :

$$
M(\mathbb{Q}) \hookrightarrow \operatorname{Isom}\left(F_{2}(\mathbb{Q}), \exp \mathfrak{f}_{2}\right) .
$$

As is explained in A, F4, a certain geometric interpretations of the GrothendieckTeichmüller groups shows that $\operatorname{Im} \Psi$ is injected in $M(\mathbb{Q})$ as bitorsors. Thus by the above theorem, $\left(\mathrm{Gal}_{\mathrm{DR}}^{\mathcal{M}}(\mathbb{Z}), \mathrm{Gal}_{\mathrm{Be}}^{\mathcal{M}}(\mathbb{Z})\right)$-bitorsor $\mathrm{Gal}_{\mathrm{Be}, \mathrm{DR}}^{\mathcal{M}}(\mathbb{Z})$ is mapped injectively to $(G R T(\mathbb{Q}), G T(\mathbb{Q}))$-bitorsor $M(\mathbb{Q})$ as bitorsors:

$$
\operatorname{Gal}_{\mathrm{Be}, \mathrm{DR}}^{\mathcal{M}}(\mathbb{Z}) \hookrightarrow M(\mathbb{Q}) .
$$

The inclusion induces the one from $\operatorname{LieGal}_{\mathrm{DR}}^{\mathcal{M}}(\mathbb{Z})_{1}$ to $G R T(\mathbb{Q})$. By Remark 1.21 we get the claim in Remark 1.14. The $G T(\mathbb{Q})$-action on $\widehat{\mathbb{Q}\left[B_{n}\right]}$ given in (1.5) induces a $\operatorname{Gal}_{\mathrm{Be}}^{\mathcal{M}}(\mathbb{Z})$-action there and $G R T(\mathbb{Q})$-action on ${\widehat{U \mathfrak{b}_{n}}}_{\text {given in }}(1.13)$ also induces a $\operatorname{Gal}_{\mathrm{DR}}^{\mathcal{M}}(\mathbb{Z})$-action there. Hence by the equivalence (1.17), $\widehat{\mathbb{Q}\left[B_{n}\right]}$ is the Betti realization of a certain mixed Tate (pro-)motive over $\operatorname{Spec} \mathbb{Z}$, while whose de Rham realization is given by ${\widehat{U \mathfrak{b}_{n}}}_{\text {. }}$.

\section{Proalgebraic tangles and Chord diagrams}

We develop the actions of the Grothendieck-Teichmüller groups on proalgebraic braids and on infinitesimal braids explained in our previous section into the ones on proalgebraic tangles and on chord diagrams by following the method indicated in $[\mathrm{KT}$. This section might be regarded as an extension of Bar-Natan's formalism [Ba3] on a relationship of the Grothendieck-Teichmüller groups with proalgebraic braids into their relationship with proalgebraic tangles.

2.1. The $G T$-action. In this subsection we give a review but with more detailed considerations on the last appendix of both [KT] and [KRT] where an interesting $G T(\mathbb{K})$-action on proalgebraic tangles and knots are briefly explained. Proalgebraic tangles and proalgebraic knots are recalled in Definition 2.3. They are shown in Proposition 2.7 to be described by proalgebraic pre-tangles (and knots) introduced by our ABC-construction in Definition 2.5 . The $G T(\mathbb{K})$-action on proalgebraic 
tangles is explained in Definition 2.9 and Proposition 2.11 The induced $G T(\mathbb{K})$ action on proalgebraic knots is discussed in Proposition 2.13. In Proposition 2.17we give a relationship of the $G T(\mathbb{K})$-action on the proalgebraic knots with the profinite $\widehat{G T}$-action on profinite knots which was constructed in our previous paper [F6].

Notation 2.1. Let $k, l \geqslant 0$. Let $\epsilon=\left(\epsilon_{1}, \ldots, \epsilon_{k}\right)$ and $\epsilon^{\prime}=\left(\epsilon_{1}^{\prime}, \ldots, \epsilon_{1}^{\prime}\right)$ be sequences (including the empty sequence $\emptyset$ ) of symbols $\uparrow$ and $\downarrow$. An (oriented) 3 tangle of type $\left(\epsilon, \epsilon^{\prime}\right)$ means a smooth embedded compact oriented one-dimensional real manifolds in $[0,1] \times \mathbb{C}$ (hence it is a finite disjoint union of embedded one-dimensional intervals and circles), whose boundaries are $\{(1,1), \ldots,(1, k),(0,1) \ldots,(0, l)\}$ such that $\epsilon_{i}$ (resp. $\epsilon_{j}^{\prime}$ ) is $\uparrow$ or $\downarrow$ if the tangle is oriented upwards or downwards at $(1, i)$ (resp. at $(0, j))$ respectively. A link is a tangle of type $(\emptyset, \emptyset)$, i.e. $k=l=0$, and a knot means a link with a single connected component. An $n$-string link 4 , a string link with $n$-components, means a tangle with $\epsilon=\epsilon^{\prime}$ and $k=l=n$ which consists of $n$-intervals connecting $(0, i)$ with $(1, i)$ for each $1 \leqslant i \leqslant n$ and no circles (cf. Figure 2.1).

We denote $\mathcal{T}$ to be the full set of isotopy classes of oriented tangles and $\mathcal{T}_{\epsilon, \epsilon^{\prime}}$ to be its subset consisting of tangles with type $\left(\epsilon, \epsilon^{\prime}\right)$. Figure 2.2 might help the readers to have a good understanding of the definition. It is easily seen that there

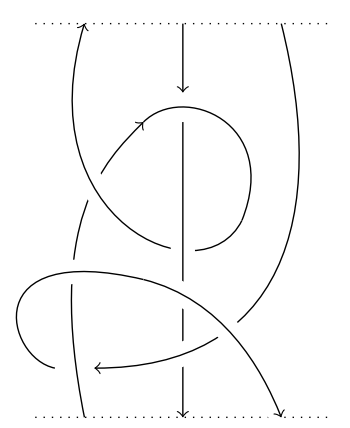

Figure 2.1. A string link

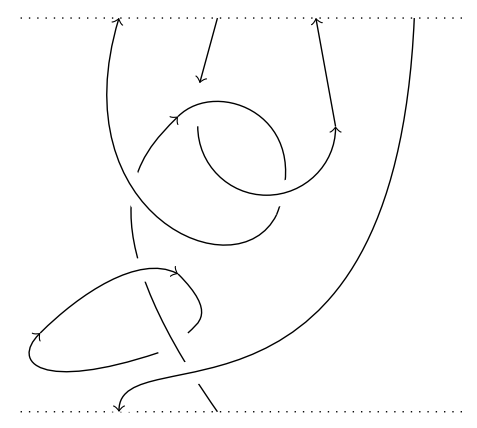

Figure 2.2. A tangle in $\mathcal{T}_{\uparrow \downarrow \uparrow \downarrow, \downarrow \uparrow}$

is a natural composition map

$$
\cdot: \mathcal{T}_{\epsilon_{1}, \epsilon_{2}} \times \mathcal{T}_{\epsilon_{2}, \epsilon_{3}} \rightarrow \mathcal{T}_{\epsilon_{1}, \epsilon_{3}}
$$

for any sequences $\epsilon_{1}, \epsilon_{2}, \epsilon_{3}$. The set $\mathcal{S} \mathcal{L}_{\epsilon}$ denotes the subspace of $\mathcal{T}_{\epsilon, \epsilon}$ consisting of string links. By the above composition $\mathcal{S} \mathcal{L}_{\epsilon}$ forms a monoid for each $\epsilon$. By putting on each $i$-th strand an orientation $\epsilon_{i}$, the pure braid group $P_{n}(n>1)$ may be regarded as a submonoid of $\mathcal{S} \mathcal{L}_{\epsilon}$ with $\epsilon=\left(\epsilon_{1}, \ldots, \epsilon_{n}\right)$. By definition $\mathcal{T}_{\emptyset, \emptyset}$ is the set of isotopy classes of (oriented) links. We denote $\mathcal{K}$ to be its subspace consisting isotopy classes of (oriented) knots. The set $\mathcal{K}$ forms a monoid by the connected sum (the knot sum)

$$
\sharp: \mathcal{K} \times \mathcal{K} \rightarrow \mathcal{K} .
$$

\footnotetext{
${ }^{3}$ We occasionally omit to mention it. Throughout the paper all tangles are assumed to be oriented.

${ }^{4}$ A string link is not a link in the sense of the previous sentence.
} 
It is a natural way to fuse two oriented knots, with an appropriate position of orientation, into one (an example is illustrated in Figure 2.3). It can be done at

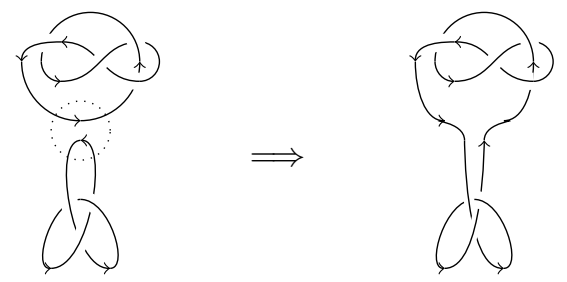

Figure 2.3. Connected sum (knot sum)

any points. Our short caution is that the connected sum $\sharp$ in (2.2) is different from the composition · in (2.1). (In fact a composition of two knots in not a knot but a link).

There is a fundamental identification between knots and long knots (string link with a single component).

Proposition 2.2. Let $\epsilon=\uparrow$ or $\downarrow$. Then the set $\mathcal{S} \mathcal{L}_{\epsilon}$ of long knots with the composition - is identified with the set $\mathcal{K}$ with the connected sum $\sharp$ by closing the two endpoints of each. Namely we have an identification of two monoids

$$
\mathrm{cl}:\left(\mathcal{S} \mathcal{L}_{\uparrow}, \cdot\right) \simeq(\mathcal{K}, \sharp) .
$$

Proof. The identification is simply obtained by combining the ends of long knots. Checking all compatibilities are easy to see.

For more on tangles, consult the standard textbook, say, CDM].

Definition 2.3 ([F6, $\overline{\mathrm{KT}}]$ ). (1) Let $\mathbb{K}\left[\mathcal{T}_{\epsilon, \epsilon^{\prime}}\right]$ be the free $\mathbb{K}$-module of finite formal sums of elements of $\mathcal{T}_{\epsilon, \epsilon^{\prime}}$. A singular oriented tangle, an 'oriented tangle' allowed to have a finite number of transversal double points (see $[\mathrm{KT}$ for datail), determines an element of $\mathbb{K}\left[\mathcal{T}_{\epsilon, \epsilon^{\prime}}\right]$ by the desingularization of each double point by the following relation

$$
X=Y-\lambda
$$

Let $\mathcal{T}_{n}(n \geqslant 0)$ be the $\mathbb{K}$-submodule of $\mathbb{K}\left[\mathcal{T}_{\epsilon, \epsilon^{\prime}}\right]$ generated by all singular oriented tangles with type $\left(\epsilon, \epsilon^{\prime}\right)$ and with $n$ double points. The descending filtration $\left\{\mathcal{T}_{n}\right\}_{n} \geqslant 0$ is called the singular filtration. The topological $\mathbb{K}$-module $\widehat{\mathbb{K}\left[\mathcal{T}_{\epsilon, \epsilon^{\prime}}\right]}$ of proalgebraic tangles of type $\left(\epsilon, \epsilon^{\prime}\right)$ means its completion with respect to the singular filtration:

$$
\widehat{\mathbb{K}\left[\mathcal{T}_{\epsilon, \epsilon^{\prime}}\right]}:=\lim _{N} \mathbb{K}\left[\mathcal{T}_{\epsilon, \epsilon^{\prime}}\right] / \mathcal{T}_{N} .
$$

By abuse of notation, we denote the induced filtration on $\widehat{\mathbb{K}\left[\mathcal{T}_{\epsilon, \epsilon^{\prime}}\right]}$ by the same symbol $\left\{\mathcal{T}_{n}\right\}_{n \geqslant 0}$. Note that there is a natural composition map

$$
\cdot: \widehat{\mathbb{K}\left[\mathcal{T}_{\epsilon_{1}, \epsilon_{2}}\right]} \times \widehat{\mathbb{K}\left[\mathcal{T}_{\epsilon_{2}, \epsilon_{3}}\right]} \rightarrow \widehat{\mathbb{K}\left[\mathcal{T}_{\epsilon_{1}, \epsilon_{3}}\right]}
$$

for any $\epsilon_{1}, \epsilon_{2}$ and $\epsilon_{3}$. We denote the collection of $\widehat{\mathbb{K}\left[\mathcal{T}_{\epsilon, \epsilon^{\prime}}\right]}$ for all $\epsilon$ and $\epsilon^{\prime}$ by $\widehat{\mathcal{T}_{\mathbb{K}}}$.

(2) Let $\mathbb{K}[\mathcal{K}]$ be the $\mathbb{K}$-submodule of $\mathbb{K}\left[\mathcal{T}_{\emptyset, \emptyset}\right]$ generated by $\mathcal{K}$. By the product

$$
\sharp: \widehat{\mathbb{K}[\mathcal{K}]} \times \widehat{\mathbb{K}[\mathcal{K}]} \rightarrow \widehat{\mathbb{K}[\mathcal{K}]}
$$


induced by the connected sum $\sharp$ in $(2.2)$ and the coproduct map $\Delta: \mathbb{K}[\mathcal{K}] \rightarrow$ $\mathbb{K}[\mathcal{K}] \otimes \mathcal{K} \mathbb{K}[\mathcal{K}]$ sending $k \mapsto k \otimes k$ and the augmentation map $\mathbb{K}[\mathcal{K}] \rightarrow \mathcal{K}$, it carries a structure of co-commutative and commutative bi-algebra. Put $\mathcal{K}_{n}:=\mathcal{T}_{n} \cap \mathbb{K}[\mathcal{K}]$ $(n \geqslant 0)$. Then $\mathcal{K}_{n}$ forms an ideal of $\mathbb{K}[\mathcal{K}]$ and the descending filtration $\left\{\mathcal{K}_{n}\right\}_{n \geqslant 0}$ is called the singular knot filtration (cf. loc.cit.). The topological commutative $\mathbb{K}$ algebra $\widehat{\mathbb{K}[\mathcal{K}]}$ of proalgebraic knots means its completion with respect to the singular knot filtration:

$$
\widehat{\mathbb{K}[\mathcal{K}]}:={\underset{\varliminf}{N}}_{\lim } \mathbb{K}[\mathcal{K}] / \mathcal{K}_{N}
$$

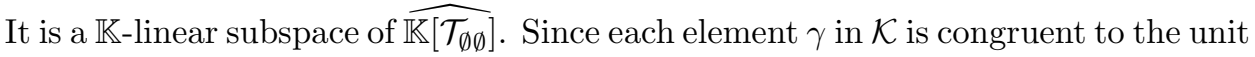
$\bigcirc$ modulo $\mathcal{K}_{1}$ due to the finiteness property of the unknotting number (cf. [CDM]), the inverse of $\gamma$ with respect to $\sharp$ always exists in $\widehat{\mathbb{K}[\mathcal{K}]}$. It defines the antipode map on $\widehat{\mathbb{K}[\mathcal{K}]}$, which yields a structure of co-commutative and commutative Hopf algebra there. Again by abuse of notation, we denote the induced filtration of $\widehat{\mathbb{K}[\mathcal{K}]}$ by the same symbol $\left\{\mathcal{K}_{n}\right\}_{n \geqslant 0}$, which is compatible with a structure of filtered Hopf algebra on $\widehat{\mathbb{K}[\mathcal{K}]}$.

(3) Proalgebraic links and proalgebraic string links can be defined in the same way. The subset $\widehat{\mathbb{K}\left[\mathcal{S L}_{\epsilon}\right]}$ of $\widehat{\mathbb{K}\left[\mathcal{T}_{\epsilon} \epsilon\right]}$ consisting of proalgebraic string links forms a non-commutative $\mathbb{K}$-algebra by the composition map (2.3).

Here is a fundamental identification in our proalgebraic setting.

Lemma 2.4. Let $\epsilon=\uparrow$ or $\downarrow$. There is an identification between two $\mathbb{K}$-algebras

$$
\mathrm{cl}:\left(\widehat{\mathbb{K}\left[\mathcal{S} \mathcal{L}_{\epsilon}\right]}, \cdot\right) \simeq(\widehat{\mathbb{K}[\mathcal{K}]}, \sharp) .
$$

which is compatible with their filtrations.

Proof. It is an immediate corollary of Proposition 2.2.

We give a piecewise construction of the above proalgebraic tangles by using proalgebraic pre-tangles introduced below.

Definition 2.5. (1) A fundamental proalgebraic (oriented) tangle means a vector belonging to an $A B C$-space, one of the following $\mathbb{K}$-linear spaces $A_{k, l}^{\epsilon}, \widehat{B_{\tau}^{\epsilon}}$ and $C_{k, l}^{\epsilon}$ for some $k, l, \epsilon, \tau$ :

$A_{k, l}^{\epsilon}:=\mathbb{K} \cdot a_{k, l}^{\epsilon}$, with $\epsilon=\left(\epsilon_{i}\right)_{i=1}^{k+l+1} \in\{\uparrow, \downarrow\}^{k} \times\{\curvearrowright, \curvearrowleft\} \times\{\uparrow, \downarrow\}^{l}(k, l=0,1,2, \ldots)$, $\widehat{B_{\tau}^{\epsilon}}:=\widehat{\mathbb{K}\left[P_{n}\right]} \cdot \tau$ with $\epsilon=\left(\epsilon_{i}\right)_{i=1}^{n} \in\{\uparrow, \downarrow\}^{n}$ and $\tau \in \mathfrak{S}_{n}(n=1,2,3,4, \ldots)$, $C_{k, l}^{\epsilon}:=\mathbb{K} \cdot c_{k, l}^{\epsilon}$, with $\epsilon=\left(\epsilon_{i}\right)_{i=1}^{k+l+1} \in\{\uparrow, \downarrow\}^{k} \times\{\smile, \smile\} \times\{\uparrow, \downarrow\}^{l}(k, l=0,1,2, \ldots)$. Here $\widehat{\mathbb{K}\left[P_{n}\right]} \cdot \tau$ stands for the coset of $\widehat{\mathbb{K}\left[B_{n}\right]} / \widehat{\mathbb{K}\left[P_{n}\right]}$ corresponding to $\tau \in \mathfrak{S}_{n}=$ $B_{n} / P_{n}$, the inverse image of $\mathbb{K} \cdot \sigma$ under the natural homomorphism $\widehat{\mathbb{K}\left[B_{n}\right]} \rightarrow \mathbb{K}\left[\mathfrak{S}_{n}\right]$. To stress that an element $b$ belongs to $\widehat{B_{\tau}^{\epsilon}}$, we occasionally denote $b^{\epsilon}$ or $(b, \epsilon)$.

For each ABC-space $V$, its source $s(V)$ and target $t(V)$, which are sequences of $\uparrow$ and $\downarrow$, are defined in a completely same way to [F6]; e.g. $s\left(\widehat{B_{\tau}^{\epsilon}}\right)=\epsilon, t\left(\widehat{B_{\tau}^{\epsilon}}\right)=\tau(\epsilon)$.

(2) A proalgebraic pre-tangle $\Gamma$ of type $\left(\epsilon, \epsilon^{\prime}\right)$ is a vector belonging to a $\mathbb{K}$-linear space which is a finite consistent (successively composable) $\mathbb{K}$-linear tensor product of ABC-spaces. Namely it is an element belonging to a $\mathbb{K}$-linear space $V_{n} \otimes \cdots V_{2} \otimes V_{1}$ for some $n$ such that $s\left(V_{i+1}\right)=t\left(V_{i}\right)$ for all $i=1,2, \ldots, n-1$ and $s\left(V_{1}\right)=\epsilon$ and 
$t\left(V_{n}\right)=\epsilon^{\prime}$. For our simplicity, we denote each of its element $\Gamma=\gamma_{n} \otimes \cdots \otimes \gamma_{2} \otimes \gamma_{1}$ with $\gamma_{i} \in V_{i}$ by $\Gamma=\gamma_{n} \cdots \gamma_{2} \cdot \gamma_{1}$. We also define $s(\Gamma):=s\left(V_{1}\right)$ and $t(\Gamma):=t\left(V_{n}\right)$. A proalgebraic pre-link $\Gamma$ is a proalgebraic pre-tangle with $s(\Gamma)=t(\Gamma)=\emptyset$. Two proalgebraic pre-tangles $\Gamma=\gamma_{n} \cdots \gamma_{2} \cdot \gamma_{1}$ and $\Gamma^{\prime}=\gamma_{m}^{\prime} \cdots \gamma_{2}^{\prime} \cdot \gamma_{1}^{\prime}$ are called composable when $s(\Gamma)=t\left(\Gamma^{\prime}\right)$. Their composition $\Gamma \cdot \Gamma^{\prime}$ is defined by $\gamma_{n} \cdots \gamma_{2} \cdot \gamma_{1} \cdot \gamma_{m}^{\prime} \cdots \gamma_{2}^{\prime} \cdot \gamma_{1}^{\prime}$.

For each ABC-space $V$, its skeleton $\mathbb{S}(V)$ is defined in a completely same way to F6. For a proalgebraic pre-tangle $\Gamma=\gamma_{n} \cdots \gamma_{2} \cdot \gamma_{1}$ with $\gamma_{i} \in V_{i}$, its skeleton $\mathbb{S}(\Gamma)$ stands for the graph of the compositions $\mathbb{S}\left(V_{n}\right) \cdots \mathbb{S}\left(V_{2}\right) \cdot \mathbb{S}\left(V_{1}\right)$ and its connected components mean the connected components of $\mathbb{S}(\Gamma)$ as graphs. A proalgebraic pre-knot is a proalgebraic pre-link with a single connected component. A proalgebraic pre-string link of type $\epsilon=\left(\epsilon_{i}\right)_{i=1}^{n}$ is a proalgebraic pre-tangle of type $(\epsilon, \epsilon)$ whose connected components consist of $n$ intervals connecting each $i$-th point on the bottom to the one on the top.

(3) Two proalgebraic pre-tangles are called isotopic when they are related by a finite number of the 6 moves replacing profinite tangles and profinite braids group $\widehat{B_{n}}$ by proalgebraic pre-tangles and proalgebraic braid algebras $\widehat{\mathbb{K}\left[B_{n}\right]}$ in (T1)-(T6) in [F6] and $c \in \widehat{\mathbb{Z}}$ by $c \in \mathbb{K}$ in (T6). (N.B. We note that $\sigma_{i}^{c}$ for $c \in \mathbb{K}$ makes sense in $\widehat{\mathbb{K}\left[B_{n}\right]}$ by the reason explained in [F6] Proof of Proposition 2.29 (2).)

(4) We denote $\mathbb{K}\left[\mathcal{T}_{\epsilon, \epsilon^{\prime}}^{\text {pre }}\right]$ to be the $\mathbb{K}$-linear space which is the quotient of the $\mathbb{K}$ span of proalgebraic pre-tangles with type $\left(\epsilon, \epsilon^{\prime}\right)$ divided by the equivalence linearly generated by the above isotopy. Note that there is a natural composition map

$$
\cdot: \mathbb{K}\left[\mathcal{T}_{\epsilon_{1}, \epsilon_{2}}^{\text {pre }}\right] \times \mathbb{K}\left[\mathcal{T}_{\epsilon_{2}, \epsilon_{3}}^{\text {pre }}\right] \rightarrow \mathbb{K}\left[\mathcal{T}_{\epsilon_{1}, \epsilon_{3}}^{\text {pre }}\right]
$$

for any $\epsilon_{1}, \epsilon_{2}$ and $\epsilon_{3}$. The subset $\mathbb{K}\left[\mathcal{S} \mathcal{L}_{\epsilon}^{\text {pre }}\right]$ of $\mathbb{K}\left[\mathcal{T}_{\epsilon, \epsilon}^{\text {pre }}\right]$ consisting of proalgebraic pre-string links of type $\epsilon$ forms a non-commutative $\mathbb{K}$-algebra by the composition map.

The symbol $\mathbb{K}\left[\mathcal{K}^{\text {pre }}\right]$ stands for the subspace of $\mathbb{K}\left[\mathcal{T}_{\emptyset, \emptyset}^{\text {pre }}\right]$ generated by proalgebraic pre-knots. It can be proven in a same way to $[\mathrm{F} 6]$ that $\mathbb{K}\left[\mathcal{K}^{\text {pre }}\right]$ inherits a structure of a commutative $\mathbb{K}$-algebra by the connected sum

$$
\sharp: \mathbb{K}\left[\mathcal{K}^{\text {pre }}\right] \times \mathbb{K}\left[\mathcal{K}^{\text {pre }}\right] \rightarrow \mathbb{K}\left[\mathcal{K}^{\text {pre }}\right] .
$$

Here for any two proalgebraic knots $K_{1}=\alpha_{m} \cdots \alpha_{1}$ and $K_{2}=\beta_{n} \cdots \beta_{1}$ with $\left(\alpha_{m}, \alpha_{1}\right)=(\curvearrowleft, \cup)$ and $\left(\beta_{n}, \beta_{1}\right)=(\curvearrowleft, \cup)$ (we may assume such presentations by (T6)), their connected sum is defined by

$$
K_{1} \sharp K_{2}:=\alpha_{m} \cdots \alpha_{2} \cdot \beta_{n-1} \cdots \beta_{1} .
$$

Again we note that $\sharp$ is different from the above composition (2.6).

(5) For a $\mathbb{K}$-linear space $V$ with $V=\widehat{B_{\tau}^{\epsilon}}$, we give its descending filtration $\left\{\mathcal{T}_{N}(V)\right\}_{N=0}^{\infty}$ of $\mathbb{K}$-linear subspaces by $\mathcal{T}_{N}(V):=I^{N}$ and for a $\mathbb{K}$-linear space $V$ with $V=A_{k, l}^{\epsilon}$ or $C_{k, l}^{\epsilon}$ we give its filtration by $\mathcal{T}_{0}(V)=V$ and $\mathcal{T}_{N}(V):=\{0\}$ for $N>0$. For a finite consistent tensor product $V=V_{n} \otimes \cdots \otimes V_{1}$ of ABC-spaces, we give its descending filtration $\left\{\mathcal{T}_{N}(V)\right\}_{N=0}^{\infty}$ with

$$
\mathcal{T}_{N}(V):=\sum_{i_{n}+\cdots+i_{1}=N} \mathcal{T}_{i_{n}}\left(V_{n}\right) \otimes \cdots \otimes \mathcal{T}_{i_{1}}\left(V_{1}\right),
$$

i.e. the $\mathbb{K}$-linear subspaces generated by the subspaces $\mathcal{T}_{i_{n}}\left(V_{n}\right) \otimes \cdots \otimes \mathcal{T}_{i_{1}}\left(V_{1}\right)$ with $i_{n}+\cdots+i_{1}=N$. For any $\epsilon$ and $\epsilon^{\prime}$, the collection of the filtrations of such consistent tensor product $V$ with $s(V)=\epsilon^{\prime}$ and $t(V)=\epsilon$ yields a filtration of $\mathbb{K}$-submodules of $\mathbb{K}\left[\mathcal{T}_{\epsilon, \epsilon^{\prime}}^{\text {pre }}\right]$, which we denote by $\left\{\mathcal{T}_{N}^{\text {pre }}\right\}_{N \geqslant 0}$. They are compatible with the composition 
map (2.6). The filtration on $\mathbb{K}\left[\mathcal{K}^{\text {pre }}\right]$ induced by the filtration of $\mathbb{K}\left[\mathcal{T}_{\emptyset, \emptyset}^{\text {pre }}\right]$ is denoted by $\left\{\mathcal{K}_{N}^{\text {pre }}\right\}_{N \geqslant 0}$. The filtration is compatible with its algebra structure given by (2.7).

Lemma 2.6. (1) For any $\epsilon$ and $\epsilon^{\prime}$ and any $N \geqslant 0$, there is an isomorphism of $\mathbb{K}$-linear spaces

$$
\mathbb{K}\left[\mathcal{T}_{\epsilon, \epsilon^{\prime}}\right] / \mathcal{T}_{N} \simeq \mathbb{K}\left[\mathcal{T}_{\epsilon, \epsilon^{\prime}}^{\text {pre }}\right] / \mathcal{T}_{N}^{\text {pre }} .
$$

(2) For any $\epsilon$ and any $N \geqslant 0$, there is an isomorphism of non-commutative $\mathbb{K}$-algebras

$$
\mathbb{K}\left[\mathcal{S} \mathcal{L}_{\epsilon}\right] / \mathcal{T}_{N} \simeq \mathbb{K}\left[\mathcal{S} \mathcal{L}_{\epsilon}^{\text {pre }}\right] / \mathcal{T}_{N}^{\text {pre }} .
$$

(3) For any $N \geqslant 0$, there is an isomorphism of commutative $\mathbb{K}$-algebras

$$
\mathbb{K}[\mathcal{K}] / \mathcal{K}_{N} \simeq \mathbb{K}\left[\mathcal{K}^{\text {pre }}\right] / \mathcal{K}_{N}^{\text {pre }} .
$$

Proof. (1) The map is obtained because the set $\mathcal{T}$ is described by the sequence of elements in the discrete sets $A, B$ and $C$ modulo the discrete version of our moves (T1)-(T6) (see F6]). Showing that it is an isomorphism is attained by the isomorphism

$$
\mathbb{K}\left[B_{n}\right] / I^{i} \simeq \widehat{\mathbb{K}\left[B_{n}\right]} / I^{i}
$$

for $i=0,1,2, \ldots$.

(2) and (3) It is a direct consequence of (1).

Here are algebraic reformulations of proalgebraic tangles and knots.

Proposition 2.7. (1) For each $\epsilon$ and $\epsilon^{\prime}$, there is an identification of filtered $K$ linear spaces

$$
\varliminf_{n} \mathbb{K}\left[\mathcal{T}_{\epsilon, \epsilon^{\prime}}^{\text {pre }}\right] / \mathcal{T}_{n}^{\text {pre }} \simeq \widehat{\mathbb{K}\left[\mathcal{T}_{\epsilon, \epsilon^{\prime}}\right]}
$$

(2) For each $\epsilon$, there is an identification of filtered non-commutative $\mathbb{K}$-algebras

$$
\varliminf_{n} \mathbb{K}\left[\mathcal{S} \mathcal{L}_{\epsilon}^{\text {pre }}\right] / \mathcal{T}_{n}^{\text {pre }} \simeq \widehat{\mathbb{K}\left[\mathcal{S} \mathcal{L}_{\epsilon}\right]} \text {. }
$$

(3) There is an identification of filtered commutative $\mathbb{K}$-algebras

$$
\varliminf_{n} \mathbb{K}\left[\mathcal{K}^{\mathrm{pre}}\right] / \mathcal{K}_{n}^{\mathrm{pre}} \simeq \widehat{\mathbb{K}[\mathcal{K}]} .
$$

Proof. It is immediate by Definition 2.3 and the above lemma.

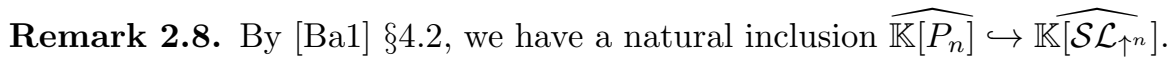

Based on the identification, the action of the Grothendieck-Teichmüller group $G T(\mathbb{K})$ on proalgebraic tangles (in $\mathrm{KRT}$, $\mathrm{KT}$ Appendix) can be explained as follows:

Definition 2.9. Let $\bar{\Gamma} \in \widehat{\mathbb{K}\left[\mathcal{T}_{\epsilon, \epsilon^{\prime}}\right]} / \mathcal{T}_{N}$ with any sequences $\epsilon, \epsilon^{\prime}$ and $N \geqslant 0$. Let $\Gamma$ be its representative in $\mathbb{K}\left[\mathcal{T}_{\epsilon, \epsilon^{\prime}}^{\text {pre }}\right]$ with a presentation $\Gamma=\gamma_{m} \cdots \gamma_{2} \cdot \gamma_{1}\left(\gamma_{j}\right.$ : fundamental proalgebraic tangle). For $\sigma=(\lambda, f) \in G T(\mathbb{K})$ with $\lambda \in \mathbb{K}^{\times}$and $f \in F_{2}(\mathbb{K})$, we define

$$
\left.\sigma(\bar{\Gamma}):=\overline{\sigma\left(\gamma_{m}\right) \cdots \sigma\left(\gamma_{2}\right) \cdot \sigma\left(\gamma_{1}\right)} \in \widehat{\mathbb{K}\left[\mathcal{T}_{\epsilon} \epsilon^{\prime}\right.}\right] / \mathcal{T}_{N} .
$$

Here $\sigma\left(\gamma_{j}\right)$ is defined in a same way to [F] as follows.

(1) If $\gamma_{j} \in A_{k, l}^{\epsilon}$, we define

$$
\sigma\left(\gamma_{j}\right):=\gamma_{j} \cdot\left(\nu_{f}\right)_{k+2}^{s\left(\gamma_{j}\right)} \cdot f_{1 \cdots k, k+1, k+2}^{s\left(\gamma_{j}\right)}
$$


Here the middle term stands for the proalgebraic tangle whose source is $s\left(\gamma_{j}\right)$ which is obtained by putting the trivial braid with $k+1$-strands on the left of $\nu_{f}$ (see below) and the trivial braid with $l$-strands on its right.

(2) If $\gamma_{j}=\left(b_{n}, \epsilon\right) \in \widehat{B_{\tau}^{\epsilon}}$ with $b_{n} \in \widehat{\mathbb{K}\left[P_{n}\right]} \cdot \tau \subset \widehat{\mathbb{K}\left[B_{n}\right]}$, we define

$$
\sigma\left(\gamma_{j}\right):=\left(\rho_{n}(\sigma)\left(b_{n}\right), \epsilon\right) .
$$

Here $\rho_{n}(\sigma)\left(b_{n}\right)$ is the image of $b_{n}$ by the action $\sigma \in G T(\mathbb{K})$ on $\widehat{\mathbb{K}\left[B_{n}\right]}$ explained in 1.1

(3) If $\gamma_{j} \in C_{k, l}^{\epsilon}$, we define

$$
\sigma\left(\gamma_{j}\right):=f_{1 \cdots k, k+1, k+2}^{-1, t\left(\gamma_{j}\right)} \cdot \gamma_{j} .
$$

The symbol $\nu_{f}^{\epsilon}(\epsilon=\uparrow, \downarrow)$ means the proalgebraic tangle in $\widehat{\mathbb{K}\left[\mathcal{T}_{\epsilon, \epsilon}\right]}$ which is a proalgebraic string link with a single strands such that $s\left(\mu_{f}^{\epsilon}\right)=t\left(\mu_{f}^{\epsilon}\right)=\epsilon$ and which is given by the inverse of $\Lambda_{f}^{\epsilon}$ with respect to the composition:

$$
\nu_{f}^{\epsilon}:=\left\{\Lambda_{f}^{\epsilon}\right\}^{-1} .
$$

Here $\Lambda_{f}^{\downarrow}$ in $\widehat{\mathbb{K}\left[\mathcal{T}_{\epsilon, \epsilon}\right]}$ represents the proalgebraic string link with a single strands given by

$$
\Lambda_{f}^{\downarrow}:=a_{1,0}^{\downarrow \curvearrowright} \cdot f^{\downarrow \uparrow \downarrow} \cdot c_{0,1}^{\bigcup \downarrow} .
$$

(cf. Figure 2.4) and $\Lambda_{f}^{\uparrow}$ is the same one obtained by reversing its all arrows.

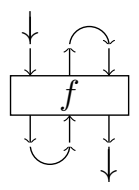

Figure 2.4. $\Lambda_{f}^{\downarrow}$

We note that the existence of the inverse of $\Lambda_{f}^{\epsilon}$ in $\widehat{\mathbb{K}\left[\mathcal{T}_{\epsilon, \epsilon}\right]}$ is immediate because $\Lambda_{f}^{\epsilon}$ is congruent to the trivial braid with a string modulo $\mathcal{T}_{1}$ and $\widehat{\mathbb{K}\left[\mathcal{T}_{\epsilon, \epsilon}\right]}$ is completed by the filtration $\left\{\mathcal{T}_{n}\right\}_{n=0}^{\infty}$.

Remark 2.10. (1) The inverse $\nu_{f}^{\epsilon}$ does not look exist in $\mathbb{K}\left[\mathcal{T}_{\epsilon, \epsilon^{\prime}}^{\mathrm{pre}}\right]$ generally. That is why we define $G T(\mathbb{K})$-action on $\widehat{\mathbb{K}\left[\mathcal{T}_{\epsilon, \epsilon^{\prime}}\right]}$ below.

(2) Our action (2.8) looks slightly different from the one given in [F6]. One of the reasons is that we deal tangles in Definition 2.9 while we discuss knots in [F6].

Proposition 2.11 ([KRT, $[\mathrm{KT}]$ ). The equation (2.8) yields a well-defined $G T(\mathbb{K})$ action on $\widehat{\mathbb{K}\left[\mathcal{T}_{\epsilon, \epsilon^{\prime}}\right]} / \mathcal{T}_{N}$ for any $\epsilon, \epsilon^{\prime}$ and any $N \geqslant 0$, and induces a well-defined $G T(\mathbb{K})$-action on $\widehat{\mathbb{K}\left[\mathcal{T}_{\epsilon, \epsilon^{\prime}}\right]}$ such that the equality

$$
\sigma\left(\Gamma \cdot \Gamma^{\prime}\right)=\sigma(\Gamma) \cdot \sigma\left(\Gamma^{\prime}\right)
$$

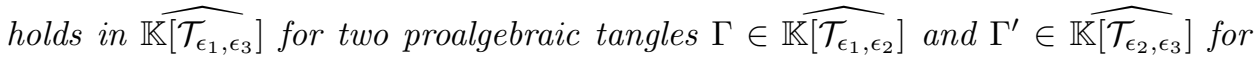
any $\epsilon_{1}, \epsilon_{2}$ and $\epsilon_{3}$. 
Proof. Though its proof can be found in loc. cit where it is explained in terms of $\mathbb{K}$-linear braided monoidal categories, we can prove in a same way to the proof of [F6] Theorem 2.38. by direct calculations.

We denote the above induced action by

$$
\rho_{\epsilon, \epsilon^{\prime}}: G T(\mathbb{K}) \rightarrow \text { Aut } \widehat{\mathbb{K}\left[\mathcal{T}_{\epsilon, \epsilon^{\prime}}\right]} .
$$

We may say that it is a generalization of the map (1.5) into the proalgebraic tangle case. As a consequence of the above proposition, we have

Proposition 2.12. For each sequence $\epsilon$, the subspace $\widehat{\mathbb{K}\left[\mathcal{S L}_{\epsilon}\right]}$ of proalgebraic string links is stable under the above $G T(\mathbb{K})$-action. The action is compatible with its noncommutative algebra structure whose product is given by the composition.

We denote the above action by

$$
\rho_{\epsilon}: G T(\mathbb{K}) \rightarrow \text { Aut } \widehat{\mathbb{K}\left[\mathcal{S} L_{\epsilon}\right]} .
$$

We will see in Theorem 3.14 that this action restricted into $G T_{1}(\mathbb{K})$ is given by inner conjugation.

Particularly by restricting our action $(2.9)$ into $\widehat{\mathbb{K}\left[\widehat{\mathcal{T}_{\emptyset, \emptyset}}\right]}$ we obtain the following.

Proposition 2.13. The subspace $\widehat{\mathbb{K}[\mathcal{K}]}$ of proalgebraic knots is stable under the above $G T(\mathbb{K})$-action. The action there is not compatible with the connected sum $\sharp$ however the equality

$$
\sigma\left(K_{1} \sharp K_{2}\right) \sharp \sigma(\bigcirc)=\sigma\left(K_{1}\right) \sharp \sigma\left(K_{2}\right)
$$

holds for any $\sigma \in G T(\mathbb{K})$ and any $K_{1}, K_{2} \in \widehat{\mathbb{K}[\mathcal{K}]}$.

Proof. Seeing that the subspace is stable can be verified by showing that connected components of skeletons of proalgebraic tangles are unchanged. The equation (2.11) can be proven in a same way to the proof of the equation (2.21) in [F6].

We denote the above action by

$$
\rho_{0}: G T(\mathbb{K}) \rightarrow \text { Aut } \widehat{\mathbb{K}\left[\mathcal{K}_{0}\right]} .
$$

Remark 2.14. We will see in Proposition 3.19 that this action is given by $\mathbb{G}_{m^{-}}$ action. We will explicitly determine in Theorem 3.27 the subspace of $\widehat{\mathbb{K}[\mathcal{K}]}$ which is invariant under the above $G T(\mathbb{K})$-action.

We note that in the proalgebraic knot setting our action above is not compatible with the product structure (2.4) due to (2.11), while in the profinite knot setting the $\widehat{G T}$-action on the group of profinite knots is compatible with the product structure as shown in [F6] Theorem 2.38.(4). In order to relate the $\widehat{G T}$-action on Frac $\widehat{\mathcal{K}}$ constructed in $[\mathrm{F} 6]$ with the above $G T(\mathbb{K})$-action on $\widehat{\mathbb{K}[\mathcal{K}]}$, we introduce the proalgebraic group $\operatorname{Frac} \mathcal{K}(\mathbb{K})$ below.

Notation 2.15. Put $\mathcal{K}(\mathbb{K})$ to be the group-like part of $\widehat{\mathbb{K}[\mathcal{K}]}$, which carries a structure of proalgebraic group. It can be checked directly that $G T(\mathbb{K})$-action on $\widehat{\mathbb{K}[\mathcal{K}]}$ is compatible with its coproduct map and its antipode map. Hence we have $G T(\mathbb{K})$-action on $\mathcal{K}(\mathbb{K})$ though it is not compatible with its product structure of $\mathcal{K}(\mathbb{K})$ due to (2.11). We denote its group of fraction by Frack $(\mathbb{K})$, which is 
the quotient space of $\mathcal{K}(\mathbb{K}) \times \mathcal{K}(\mathbb{K})$ by the equivalent relations $(r, s) \approx\left(r^{\prime}, s^{\prime}\right)$ if $r \sharp s^{\prime} \sharp t=r^{\prime} \sharp s \sharp t$ holds in $\mathcal{K}(\mathbb{K})$ for some $t \in \mathcal{K}(\mathbb{K})$.

Lemma 2.16. The induced $G T(\mathbb{K})$-action on $\operatorname{Frac} \mathcal{K}(\mathbb{K})$ is compatible its group structure, i.e.

$$
\sigma(e)=e, \quad \sigma(x \sharp y)=\sigma(x) \sharp \sigma(y), \quad \sigma\left(\frac{1}{x}\right)=\frac{1}{\sigma(x)}
$$

for any $\sigma \in G T(\mathbb{K})$ and $x, y \in \operatorname{Frac} \mathcal{K}(\mathbb{K})$. Here $e=\varnothing / \circlearrowleft$.

Proof. Let $x=r_{1} / s_{1}$ and $y=r_{2} / s_{2}$. Then by (2.11) it is easy to see

$$
\begin{aligned}
\sigma(x \sharp y) & =\sigma\left(\frac{r_{1} \sharp r_{2}}{s_{1} \sharp s_{2}}\right)=\frac{\sigma\left(r_{1} \sharp r_{2}\right)}{\sigma\left(s_{1} \sharp s_{2}\right)}=\frac{\sigma\left(r_{1} \sharp r_{2}\right) \sharp \sigma(\bigcirc)}{\sigma\left(s_{1} \sharp s_{2}\right) \sharp \sigma(\bigcirc)}=\frac{\sigma\left(r_{1}\right) \sharp \sigma\left(r_{2}\right)}{\sigma\left(s_{1}\right) \sharp \sigma\left(s_{2}\right)} \\
& =\frac{\sigma\left(r_{1}\right)}{\sigma\left(s_{1}\right)} \sharp \frac{\sigma\left(r_{2}\right)}{\sigma\left(s_{2}\right)}=\sigma\left(\frac{r_{1}}{s_{1}}\right) \sharp \sigma\left(\frac{r_{2}}{s_{2}}\right)=\sigma(x) \sharp \sigma(y), \\
\sigma\left(\frac{1}{x}\right) \sharp \sigma(x) & =\sigma\left(\frac{s_{1}}{r_{1}}\right) \sharp \sigma\left(\frac{r_{1}}{s_{1}}\right)=\frac{\sigma\left(s_{1}\right)}{\sigma\left(r_{1}\right)} \sharp \frac{\sigma\left(r_{1}\right)}{\sigma\left(s_{1}\right)}=\frac{\sigma\left(s_{1}\right) \sharp \sigma\left(r_{1}\right)}{\sigma\left(r_{1}\right) \sharp \sigma\left(s_{1}\right)}=\frac{\sigma\left(r_{1}\right) \sharp \sigma\left(s_{1}\right)}{\sigma\left(r_{1}\right) \sharp \sigma\left(s_{1}\right)}=\frac{\bigcirc}{\bigcirc}=e .
\end{aligned}
$$

In [F6], the profinite $\widehat{G T}$-action on Frac $\widehat{\mathcal{K}}$ was constructed. A relationship of the action with the above constructed $G T(\mathbb{K})$-action on $\mathcal{K}(\mathbb{K})$ is explicitly given below.

Proposition 2.17. For each prime $l$, there is a natural group homomorphism

$$
\operatorname{Frac} \widehat{\mathcal{K}} \rightarrow \operatorname{Frac} \mathcal{K}\left(\mathbb{Q}_{l}\right) \text {. }
$$

The $\widehat{G T}$-action on Frac $\widehat{\mathcal{K}}$ constructed in $[\mathrm{F} 6]$ is compatible with the above $G T\left(\mathbb{Q}_{l}\right)$ action on $\mathcal{K}\left(\mathbb{Q}_{l}\right)$ under the maps (1.6) and (2.13).

Proof. The map (2.13) is naturally induced by the map in [F6] Proposition 2.30. It follows from our construction that the map is compatible with two actions.

2.2. The GRT-action. In this subsection, we establish an 'infinitesimal' counterpart of the $G T(\mathbb{K})$-action on proalgebraic tangles given in the previous subsection. Infinitesimal tangles (and knots) are introduced in Definition 2.19 as an infinitesimal counterpart of proalgebraic tangles (and knots). (It will be shown in next subsection that this notion coincides with the notion of chord diagrams.) A consistent $G R T(\mathbb{K})$-action there is established in Proposition 2.23.

Definition 2.18. (1) A fundamental infinitesimal tangle means a vector belonging to one of the following $\mathbb{K}$-linear spaces (let us again call them ABC-spaces): $A_{k, l}^{\epsilon}$, $\widehat{I B_{\tau}^{\epsilon}}$ and $C_{k, l}^{\epsilon}$ for some $k, l, \epsilon, \tau$ :

$A_{k, l}^{\epsilon}:=\mathbb{K} \cdot a_{k, l}^{\epsilon}$, with $\epsilon=\left(\epsilon_{i}\right)_{i=1}^{k+l+1} \in\{\uparrow, \downarrow\}^{k} \times\{\curvearrowright, \curvearrowleft\} \times\{\uparrow, \downarrow\}^{l}(k, l=0,1,2, \ldots)$, $\widehat{I B_{\tau}^{\epsilon}}:=\widehat{U \mathfrak{p}_{n}} \cdot \tau$ with $\epsilon=\left(\epsilon_{i}\right)_{i=1}^{n} \in\{\uparrow, \downarrow\}^{n}$ and $\tau \in \mathfrak{S}_{n}(n=1,2,3,4, \ldots)$,

$C_{k, l}^{\epsilon}:=\mathbb{K} \cdot c_{k, l}^{\epsilon}$, with $\epsilon=\left(\epsilon_{i}\right)_{i=1}^{k+l+1} \in\{\uparrow, \downarrow\}^{k} \times\{\cup, \sim\} \times\{\uparrow, \downarrow\}^{l}(k, l=0,1,2, \ldots)$.

Here $\widehat{U \mathfrak{p}_{n}} \cdot \tau$ stands for the coset of $\widehat{U \mathfrak{b}_{n}} / \widehat{U \mathfrak{p p}_{n}}$ corresponding to $\tau \in \mathfrak{S}_{n}=B_{n} / P_{n}$. To stress that an element $b$ belongs to $\widehat{I B_{\tau}^{\epsilon}}$, we occasionally denote $b^{\epsilon}$ or $(b, \epsilon)$.

For each above space $V$, its source $s(V)$ and target $t(V)$, are defined in a completely same way to Definition 2.5 . 
(2) An infinitesimal pre-tangle $D$ of type $\left(\epsilon, \epsilon^{\prime}\right)$ is defined in a same way to Definition 2.5. Namely it is $D=d_{n} \cdots d_{2} \cdot d_{1}$ where each $d_{i}$ is an infinitesimal fundamental tangle, a vector belonging to $V_{i}$, one of the above ABC-spaces, such that $s\left(V_{i+1}\right)=t\left(V_{i}\right)$ for all $i=1,2, \ldots, n-1$ and $s\left(V_{1}\right)=\epsilon$ and $t\left(V_{n}\right)=\epsilon^{\prime}$. We also define $s(D):=s\left(V_{1}\right)$ and $t(D):=t\left(V_{n}\right)$. An infinitesimal pre-link $D$ is an infinitesimal pre-tangle with $s(D)=t(D)=\emptyset$. Two infinitesimal pre-tangles $D=d_{n} \cdots d_{2} \cdot d_{1}$ and $D^{\prime}=d_{m}^{\prime} \cdots d_{2}^{\prime} \cdot d_{1}^{\prime}$ are called composable when $s(D)=t\left(D^{\prime}\right)$ and their composition $D \cdot D^{\prime}$ is defined by $d_{n} \cdots d_{2} \cdot d_{1} \cdot d_{m}^{\prime} \cdots d_{2}^{\prime} \cdot d_{1}^{\prime}$.

For an infinitesimal pre-tangle $D$ its skeleton $\mathbb{S}(D)$ and its connected components can be defined in the same way to Definition 2.5. An infinitesimal pre-knot is an infinitesimal pre-link with a single connected component. An infinitesimal prestring link of type $\epsilon=\left(\epsilon_{i}\right)_{i=1}^{n}$ is an infinitesimal pre-tangle of type $(\epsilon, \epsilon)$ consisting of $n$ connected components whose each $i$-th component connect $i$-th point on the bottom to the one on the top.

(3) Two infinitesimal pre-tangles are called isotopic when they are related by a finite number of the 6 moves (IT1)-(IT6). Here (IT1)-(IT5) are the moves replacing profinite tangles and profinite braids group $\widehat{B_{n}}$ by infinitesimal pre-tangles and infinitesimal braid algebras ${\widehat{U \mathfrak{b}_{n}}}_{\mathrm{in}}$ (T1)-(T5) in [F6] and (IT6) is an 'infinitesimal' variant of (T6), which is stated below.

(IT6): For $\alpha \in \mathbb{K}, c_{k, l}^{\epsilon} \in C_{k, l}^{\epsilon}$ and $t_{k+1, k+2} \cdot \tau \in \widehat{I B_{\tau}^{\prime}}$ with $\tau=\tau_{k+1, k+2} \in \mathfrak{S}_{k+l+2}$ (the switch of $k+1$ and $k+2$ ) and $t\left(C_{k, l}^{\epsilon}\right)=\epsilon^{\prime}$

$$
\exp \left\{\alpha t_{k+1, k+2}\right\} \cdot \tau_{k+1, k+2} \cdot c_{k, l}^{\epsilon}=c_{k, l}^{\bar{\epsilon}}
$$

where $\bar{\epsilon}$ is the sequence obtained by revering the $(k+1)$-st and $(k+2)$-nd arrows. And for $\alpha \in \mathbb{K}, a_{k, l}^{\epsilon} \in A_{k, l}^{\epsilon}$ and $\tau \cdot t_{k+1, k+2} \in \widehat{I B_{\tau}^{\epsilon^{\prime}}}$ with $\tau=\tau_{k+1, k+2} \in \mathfrak{S}_{k+l+2}$ and $s\left(A_{k, l}^{\epsilon}\right)=\tau\left(\epsilon^{\prime}\right)$

$$
a_{k, l}^{\epsilon} \cdot \tau_{k+1, k+2} \cdot \exp \left\{\alpha t_{k+1, k+2}\right\}=a_{k, l}^{\bar{\epsilon}} \cdot
$$

where $\bar{\epsilon}$ is the sequence obtained by revering the $(k+1)$-st and $(k+2)$-nd arrows. Figure 2.5 depicts the moves. (N.B. We note that $\exp \left\{\alpha t_{k+1, k+2}\right\}$ for $\alpha \in \mathbb{K}$ makes sense in $\left.\widehat{U \mathfrak{p}}_{k+l+2} \cdot\right)$
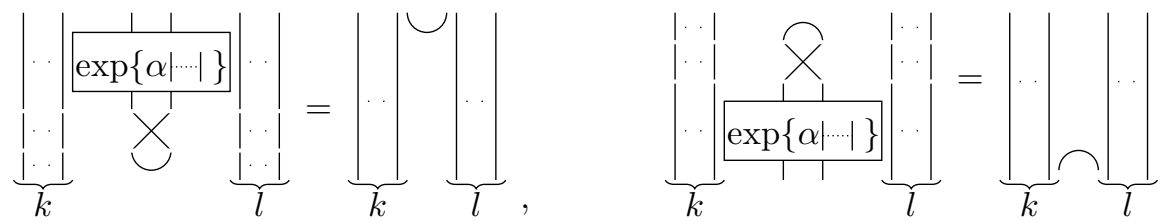

FiguRE 2.5. (IT6)

(4) We denote $\mathbb{K}\left[\mathcal{I T}_{\epsilon, \epsilon^{\prime}}^{\text {pre }}\right]$ to be the $\mathbb{K}$-linear space which is the quotient of the $\mathbb{K}$ span of infinitesimal pre-tangles with type $\left(\epsilon, \epsilon^{\prime}\right)$ divided by the equivalence linearly generated by the above isotopy. Note that there is a natural composition map

$$
\cdot: \mathbb{K}\left[\mathcal{I} \mathcal{T}_{\epsilon_{1}, \epsilon_{2}}^{\text {pre }}\right] \times \mathbb{K}\left[\mathcal{I} \mathcal{T}_{\epsilon_{2}, \epsilon_{3}}^{\text {pre }}\right] \rightarrow \mathbb{K}\left[\mathcal{I} \mathcal{T}_{\epsilon_{1}, \epsilon_{3}}^{\text {pre }}\right]
$$

for any $\epsilon_{1}, \epsilon_{2}$ and $\epsilon_{3}$. The subspace $\mathbb{K}\left[\mathcal{I} \mathcal{S} \mathcal{L}_{\epsilon}^{\text {pre }}\right]$ of $\mathbb{K}\left[\mathcal{I} \mathcal{T}_{\epsilon, \epsilon}^{\text {pre }}\right]$ consisting of infinitesimal pre-string links forms a non-commutative $\mathbb{K}$-algebras by the composition (2.14). 
The symbol $\mathbb{K}\left[\mathcal{I} \mathcal{K}^{\text {pre }}\right]$ stands for the subspace of $\mathbb{K}\left[\mathcal{I} \mathcal{T}_{\emptyset, \emptyset}^{\text {pre }}\right]$ generated by infinitesimal pre-knots. As in Definition 2.5 it inherits a structure of a commutative $\mathbb{K}$-algebra by the connected sum (which can be defined in the same way to [F6])

$$
\sharp: \mathbb{K}\left[\mathcal{I} \mathcal{K}^{\mathrm{pre}}\right] \times \mathbb{K}\left[\mathcal{I K}^{\mathrm{pre}}\right] \rightarrow \mathbb{K}\left[\mathcal{I} \mathcal{K}^{\mathrm{pre}}\right] .
$$

Again we note that $\sharp$ is different from the above composition (2.14).

(5) For a $\mathbb{K}$-linear space $V$ with $V=A_{k, l}^{\epsilon}$ or $C_{k, l}^{\epsilon}$ we give its descending filtration of $\mathbb{K}$-linear subspaces by $\mathcal{I T}_{0}(V)=V$ and $\mathcal{I T}_{N}(V):=\{0\}$ for $N>0$, and when $V=\widehat{I B_{\tau}^{\epsilon}}$, we give its descending filtration $\left\{\mathcal{I T}_{N}(V)\right\}_{N=0}^{\infty}$ such that $\mathcal{I T}_{N}(V)$ is the $\mathbb{K}$-linear subspace topologically generated by elements whose degrees are greater than or equal to $N$.

By the method indicated in Definition 2.5, for any sequences $\epsilon$ and $\epsilon^{\prime}, \mathbb{K}\left[\mathcal{I} \mathcal{T}_{\epsilon, \epsilon^{\prime}}^{\mathrm{pre}}\right]$ is inherited a filtration of its $\mathbb{K}$-submodules which we denote by $\left\{\mathcal{I}_{N}^{\text {pre }}\right\}_{N \geqslant 0}$. They are compatible with the composition map (2.14). The filtration on $\mathbb{K}\left[\mathcal{I} \mathcal{K}^{\text {pre }}\right]$ induced by the filtration of $\mathbb{K}\left[\mathcal{I} \mathcal{T}_{\emptyset, \emptyset}^{\text {pre }}\right]$ is denoted by $\left\{\mathcal{I} \mathcal{K}_{N}^{\text {pre }}\right\}_{N \geqslant 0}$. The filtration is compatible with its algebra structure given by (2.15).

Definition 2.19. (1) An infinitesimal tangle with type $\left(\epsilon, \epsilon^{\prime}\right)$ is an element of

$$
\mathbb{K} \widehat{\left[\mathcal{I} \mathcal{T}_{\epsilon, \epsilon^{\prime}}\right]}:=\varliminf_{n} \mathbb{K}\left[\mathcal{I} \mathcal{T}_{\epsilon, \epsilon^{\prime}}^{\text {pre }}\right] / \mathcal{I} \mathcal{T}_{n}^{\text {pre }} .
$$

(2) An infinitesimal string link with type $\epsilon$ is an element of

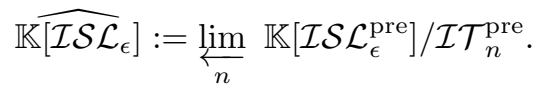

(3) An infinitesimal knot is an element of the following completed $\mathbb{K}$-algebra

$$
\widehat{\mathbb{K}[\mathcal{I K}]}:=\lim _{n} \mathbb{K}\left[\mathcal{I} \mathcal{K}^{\mathrm{pre}}\right] / \mathcal{I} \mathcal{K}_{n}^{\mathrm{pre}}
$$

We note that $\mathbb{K} \widehat{\left[\mathcal{I T}_{\epsilon, \epsilon^{\prime}}\right]}$ for $\epsilon, \epsilon^{\prime}$ is inherited a composition product · by (2.14)

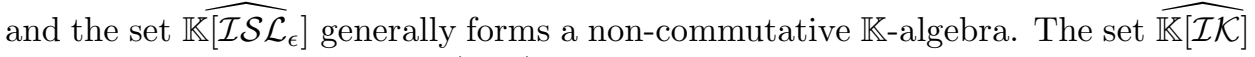
is inherited a connected $\sharp$ by $(2.15)$ and forms a commutative $\mathbb{K}$-algebra.

Remark 2.20. By Ba1 $\S 4.2$, we have a natural inclusion $\widehat{U \mathfrak{p}_{n}} \hookrightarrow \mathbb{K}\left[\widehat{\mathcal{I S \mathcal { L } _ { \uparrow n }}}\right]$.

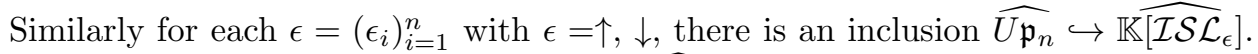
We denote the image of each element $h \in \widehat{\mathbb{U p}_{n}}$ by $h^{\epsilon}$.

As an analogue of Lemma 2.4 we have

Lemma 2.21. Let $\epsilon=\uparrow$ or $\downarrow$. There is an identification of two algebras

$$
\mathrm{cl}:\left(\widehat{\mathbb{K}\left[\widehat{\mathcal{I S L}}_{\epsilon}\right]}, \cdot\right) \simeq(\widehat{\mathbb{K}[\mathcal{I K K}]}, \sharp)
$$

which is compatible with their filtrations.

Based on the identification, the action of the graded Grothendieck-Teichmüller group $G R T(\mathbb{K})$ on infinitesimal tangles is constructed as follows:


Let $D$ be its representative in $\mathbb{K}\left[\mathcal{I} \mathcal{T}_{\epsilon, \epsilon^{\prime}}^{\text {pre }}\right]$ with a presentation $D=d_{m} \cdots d_{2} \cdot d_{1}\left(d_{j}\right.$ : 
fundamental infinitesimal tangle). For $\sigma=(c, g) \in G R T(\mathbb{K})$, hence $c \in \mathbb{K}^{\times}$and $g \in \exp \mathfrak{f}_{2}$, we define

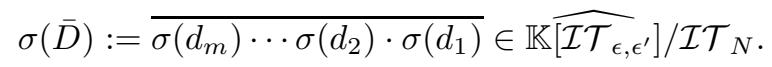

with $\sigma\left(d_{j}\right)$ given below.

(1) when $d_{j} \in A_{k, l}^{\epsilon}$, we define

$$
\sigma\left(d_{j}\right):=d_{j} \cdot\left(\nu_{g}\right)_{k+2}^{s\left(d_{j}\right)} \cdot g_{1 \cdots k, k+1, k+2}^{s\left(d_{j}\right)}
$$

Here the middle term stands for the infinitesimal tangle whose source is $s\left(d_{j}\right)$ which is obtained by putting the trivial infinitesimal braid with $k+1$-strands on the left of $\nu_{g}$ (see below) and the trivial infinitesimal braid with $l$-strands on its right.

(2) when $d_{j}=\left(b_{n}, \epsilon\right) \in \widehat{I B_{\tau}^{\epsilon}}$ with $b_{n} \in \widehat{U \mathfrak{p}_{n}} \cdot \tau \subset \widehat{U \mathfrak{b}_{n}}$, we define

$$
\sigma\left(d_{j}\right):=\left(\rho_{n}(\sigma)\left(b_{n}\right), \epsilon\right) .
$$

Here $\rho_{n}(\sigma)\left(b_{n}\right)$ is the image of $b_{n}$ by the action $\sigma \in G R T(\mathbb{K})$ on $\widehat{U \mathfrak{b}_{n}}$ explained in 1.2

(3) when $d_{j} \in C_{k, l}^{\epsilon}$, we define

$$
\sigma\left(d_{j}\right):=g_{1 \cdots k, k+1, k+2}^{-1, t\left(d_{j}\right)} \cdot d_{j} .
$$

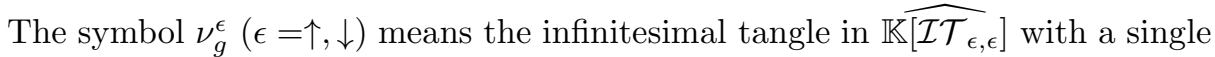
connected component such that $s\left(\mu_{g}^{\epsilon}\right)=t\left(\mu_{g}^{\epsilon}\right)=\epsilon$ and which is given by the inverse of $\Lambda_{g}^{\epsilon}$ with respect to the composition:

$$
\nu_{g}^{\epsilon}:=\left\{\Lambda_{g}^{\epsilon}\right\}^{-1} .
$$

Here $\Lambda_{g}^{\downarrow}$ in $\widehat{\mathbb{K}\left[\mathcal{I T}_{\epsilon, \epsilon}\right]}$ represents the infinitesimal string link with a single strands given by

$$
\Lambda_{g}^{\downarrow}:=a_{1,0}^{\downarrow \curvearrowright} \cdot g^{\downarrow \uparrow \downarrow} \cdot c_{0,1}^{\downarrow \downarrow} \cdot
$$

(which can be depicted as the picture in Figure 2.4 replacing $f$ by $g$ ) and $\Lambda_{g}^{\uparrow}$ is the same one obtained by reversing its all arrows.

We note that the existence of the inverse of $\Lambda_{g}^{\epsilon}$ in $\widehat{\mathbb{K}\left[\mathcal{I T}_{\epsilon, \epsilon}\right]}$ is immediate because $\Lambda_{g}^{\epsilon}$ is congruent to the unit in $\widehat{\mathbb{K}\left[\mathcal{I \mathcal { T }}_{\epsilon, \epsilon}\right]}$ modulo $\mathcal{T}_{1}$ and $\overline{\mathbb{K}\left[\mathcal{I T}_{\epsilon, \epsilon}\right]}$ is completed by the filtration $\left\{\mathcal{T}_{n}\right\}_{n=0}^{\infty}$.

Proposition 2.23. (1). The equation (2.16) yields a well-defined $G R T(\mathbb{K})$-action on $\mathbb{K}\left[\widehat{\mathcal{I} \mathcal{T}_{\epsilon, \epsilon^{\prime}}}\right] / \mathcal{I} \mathcal{T}_{N}$ for any $\epsilon, \epsilon^{\prime}$ and any $N \geqslant 0$, and induces a well-defined $G R T(\mathbb{K})$ -

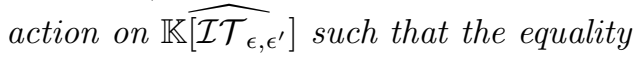

$$
\sigma\left(D \cdot D^{\prime}\right)=\sigma(D) \cdot \sigma\left(D^{\prime}\right)
$$

holds in $\mathbb{K}\left[\widehat{\mathcal{I} \mathcal{T}_{\epsilon_{1}}, \epsilon_{3}}\right]$ for two infinitesimal tangles $D \in \mathbb{K}\left[\widehat{\mathcal{I} \mathcal{T}_{\epsilon_{1}}, \epsilon_{2}}\right]$ and $D^{\prime} \in \mathbb{K}\left[\widehat{\mathcal{I} \mathcal{T}_{\epsilon_{2}}, \epsilon_{3}}\right]$ for any $\epsilon_{1}, \epsilon_{2}$ and $\epsilon_{3}$.


stable under the above $G R T(\mathbb{K})$-action. The action is compatible with its algebra structure whose product is given by the composition. 
(3) The subspace $\widehat{\mathbb{K}[\mathcal{I K}]}$ of infinitesimal knots is stable under the above $G R T(\mathbb{K})$ action. The action there is not compatible with the connected sum $\sharp$ however the equality

$$
\sigma\left(D_{1} \sharp D_{2}\right) \sharp \sigma(\circlearrowleft)=\sigma\left(D_{1}\right) \sharp \sigma\left(D_{2}\right)
$$

holds for any $\sigma \in G R T(\mathbb{K})$ and any $D_{1}, D_{2} \in \widehat{\mathbb{K}[\mathcal{I K}]}$.

Proof. Proof can be done in a completely same way to the proof of Proposition 2.11 and 2.13 ,

We denote the above induced actions respectively by

$$
\begin{aligned}
& \rho_{\epsilon, \epsilon^{\prime}}: G R T(\mathbb{K}) \rightarrow \text { Aut } \widehat{\mathbb{K}} \widehat{\left[\mathcal{I} \mathcal{T}_{\epsilon, \epsilon^{\prime}}\right]},
\end{aligned}
$$

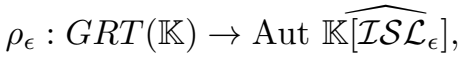

$$
\begin{aligned}
& \rho_{0}: G R T(\mathbb{K}) \rightarrow \text { Aut } \widehat{\mathbb{K}[\mathcal{I} \mathcal{K}]} .
\end{aligned}
$$

We may say that the map (2.18) is a generalization of the map (1.13) into the infinitesimal tangle case. We will see that the action (2.19) restricted into $G R T_{1}(\mathbb{K})$ is given by inner conjugation in Theorem 3.10 and that the action (2.20) is given by $\mathbb{G}_{m}$-action in Proposition 3.19 .

2.3. Associators. In this subsection we reformulate the isomorphism given in $\mathrm{KT}$ Theorem 2.4 (also shown in [Ba2, C, [KT, LM1, P]) in our terminologies of proalgebraic and infinitesimal tangles. It is shown that each associator gives an isomorphism between the system of proalgebraic tangles and the system of infinitesimal tangles in Proposition 2.25. Proposition 2.28 shows an equivalence of the notion of infinitesimal tangles and the notion of chord diagrams.

Similarly to our previous subsections, such an isomorphism is constructed piecewise.

Definition 2.24. Let $\bar{\Gamma} \in \widehat{\mathbb{K}\left[\mathcal{T}_{\epsilon, \epsilon^{\prime}}\right]} / \mathcal{T}_{N}$ with any sequences $\epsilon, \epsilon^{\prime}$ and $N \geqslant 0$. Let $\Gamma$ be its representative in $\mathbb{K}\left[\mathcal{T}_{\epsilon, \epsilon^{\prime}}^{\text {pre }}\right]$ with a presentation $\Gamma=\gamma_{m} \cdots \gamma_{2} \cdot \gamma_{1}\left(\gamma_{j}\right.$ : fundamental proalgebraic tangle). For $p=(\mu, \varphi) \in M(\mathbb{K})$, hence $\mu \in \mathbb{K}^{\times}$and $\varphi \in \exp \mathfrak{f}_{2}$, we define

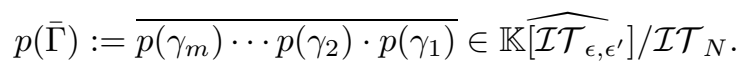

with $p\left(\gamma_{j}\right)$ given below.

(1) when $\gamma_{j} \in A_{k, l}^{\epsilon}$, we define

$$
p\left(\gamma_{j}\right):=\gamma_{j} \cdot\left(\nu_{\varphi}\right)_{k+2}^{s\left(\gamma_{j}\right)} \cdot \varphi_{1 \cdots k, k+1, k+2}^{s\left(\gamma_{j}\right)} .
$$

Here $\nu_{\varphi}$ is the infinitesimal string link defined in Definition 2.22.

(2) when $\gamma_{j}=\left(b_{n}, \epsilon\right) \in \widehat{B_{\tau}^{\epsilon}}$ with $b_{n} \in \widehat{\mathbb{K}\left[P_{n}\right]} \cdot \tau \subset \widehat{\mathbb{K}\left[B_{n}\right]}$, we define

$$
p\left(\gamma_{j}\right):=\left(\rho_{n}(p)\left(b_{n}\right), \epsilon\right) .
$$

Here $\rho_{n}(p)\left(b_{n}\right) \in \widehat{\widehat{U b}_{n}}$ is the image of $b_{n}$ by the map given in Proposition 1.19

(3) when $\gamma_{j} \in C_{k, l}^{\epsilon}$, we define

$$
p\left(\gamma_{j}\right):=\varphi_{1 \cdots k, k+1, k+2}^{-1, t\left(\gamma_{j}\right)} \cdot \gamma_{j} .
$$

As an analogue of Proposition 2.11 and 2.23 in this subsection, we have the following: 
Proposition 2.25. (1) For each $p=(\mu, \varphi) \in M(\mathbb{K})$, the equation (2.21) yields a well-defined isomorphism of $\mathbb{K}$-linear spaces



for any $\epsilon$ and $\epsilon^{\prime}$ and $N \geqslant 0$.

(2) This induces an isomorphism of $\mathbb{K}$-linear spaces

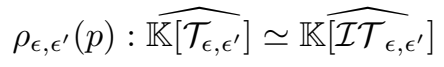

such that the equality

$$
\rho_{\epsilon_{1}, \epsilon_{3}}(p)\left(\Gamma \cdot \Gamma^{\prime}\right)=\rho_{\epsilon_{1}, \epsilon_{2}}(p)(\Gamma) \cdot \rho_{\epsilon_{2}, \epsilon_{3}}(p)\left(\Gamma^{\prime}\right)
$$

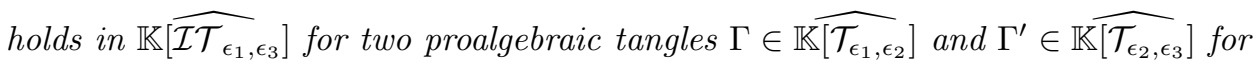
any $\epsilon_{1}, \epsilon_{2}$ and $\epsilon_{3}$.

(3) Restriction of the map (2.22) into proalgebraic string links of type $\epsilon$ yields an isomorphism

$$
\rho_{\epsilon}(p): \widehat{\mathbb{K}\left[\mathcal{S L}_{\epsilon}\right]} \simeq \widehat{\mathbb{K}\left[\widehat{\mathcal{I S L}}_{\epsilon}\right]} .
$$

It is compatible with non-commutative product structures of both algebra given by the compositions.

(4) Restriction of the map (2.22) into proalgebraic knots yields an isomorphism

$$
\rho_{0}(p): \widehat{\mathbb{K}[\mathcal{K}]} \simeq \widehat{\mathbb{K}[\mathcal{I} \mathcal{K}]}
$$

It is not compatible with the commutative product structure given by the connected sum $\sharp$ however the equality

$$
\rho_{0}(p)\left(K_{1} \sharp K_{2}\right) \sharp \rho_{0}(p)(\bigcirc)=\rho_{0}(p)\left(K_{1}\right) \sharp \rho_{0}(p)\left(K_{2}\right)
$$

holds in $\widehat{\mathbb{K}[\mathcal{I} \mathcal{K}]}$ for any $K_{1}, K_{2} \in \widehat{\mathbb{K}[\mathcal{K}]}$.

Proof. (1) Firstly we have to show that Definition 2.24 makes sense, that is, the map $\rho_{\epsilon, \epsilon^{\prime}}^{N}(p)$ is well-defined. It is enough to prove the equality $\rho_{\epsilon, \epsilon^{\prime}}^{N}(p)\left(\overline{\Gamma_{1}}\right)=\rho_{\epsilon, \epsilon^{\prime}}^{N}(p)\left(\overline{\Gamma_{2}}\right)$ for $\Gamma_{1}$ and $\Gamma_{2} \in \widehat{\mathbb{K}\left[\mathcal{T}_{\epsilon, \epsilon^{\prime}}\right]}$ when $\Gamma_{1}$ is obtained from $\Gamma_{2}$ by a single operation of one of the moves (T1)-(T6). This can be proved in a completely same way to the proof of F6 Theorem 2.38.(1).

Secondly we prove that the map $\rho_{\epsilon, \epsilon^{\prime}}^{N}(p)$ is isomorphic. This is achieved by considering the $\mathbb{K}$-linear map

$$
S_{a}: \mathcal{I T}_{a} / \mathcal{I T}_{a+1} \rightarrow \mathcal{T}_{a} / \mathcal{I T}_{a+1}
$$

for $a=0,1,2, \ldots, N-1$. It is a map sending each $\Gamma=\gamma_{m} \cdots \gamma_{1}$ with each $\gamma_{i}$ belonging to one $V_{i}$ of the ABC-spaces to $S_{a}(\Gamma):=S_{a}\left(\gamma_{m}\right) \cdots S_{a}\left(\gamma_{1}\right)$ with $S_{a}\left(\gamma_{i}\right)=\gamma_{i}$ when $V_{i}=A_{k, l}^{\epsilon}$ or $C_{k, l}^{\epsilon}$ for some $k, l, \epsilon$, and $S_{a}\left(\gamma_{i}\right)=\rho_{n}(p)^{-1}\left(\gamma_{i}\right)$ when $V_{i}=\widehat{I B_{\epsilon}^{\tau}}$ for some $\tau \in \mathfrak{S}_{n}$ (some $n \geqslant 1$ ) and $\epsilon$. Here $\rho_{n}(p): \widehat{\mathbb{K}\left[B_{n}\right]} \rightarrow \widehat{U \mathfrak{b b}_{n}}$ is the isomorphism given by (1.16). The well-definedness of $S_{a}$ can be checked directly. To see the compatibility for the move (IT6), we need to use the congruence $\rho_{n}(p)^{-1}\left(t_{k+1, k+2}\right) \equiv \sigma_{k+1}-\sigma_{k+1}^{-1}\left(\bmod I^{1}\right)$. By the construction, $S_{a}$ is isomorphic. By checking that $S_{a}$ gives an right inverse of

$$
\left.\rho_{\epsilon, \epsilon^{\prime}}^{a+1}(p)\right|_{\mathcal{T}_{a}}: \mathcal{T}_{a} / \mathcal{I T}_{a+1} \rightarrow \mathcal{I T}_{a} / \mathcal{I T}_{a+1},
$$

inductively we get that $\rho_{\epsilon, \epsilon^{\prime}}^{a+1}(p)$ is isomorphic. 
(2) Since both filtration $\left\{\mathcal{T}_{n}\right\}_{n=0}^{\infty}$ and $\left\{\mathcal{I} \mathcal{T}_{n}\right\}_{n=0}^{\infty}$ are compatible with the isomorphism $\rho_{\epsilon, \epsilon^{\prime}}^{N}(p)$, the isomorphism (2.22) is obtained. Checking the equality of the composition is immediate to see.

(3) It can be proved by the same arguments of the proof of Proposition 2.12,

(4) The statements follow from the same arguments given in the proof of Proposition 2.13 .

The above isomorphism (2.22) is compatible with both $G T(\mathbb{K})$-action on $\widehat{\mathbb{K}\left[\mathcal{T}_{\epsilon, \epsilon^{\prime}}\right]}$ and $G R T(\mathbb{K})$-action on $\widehat{\mathbb{K}} \widehat{\left[\mathcal{I} \mathcal{T}_{\epsilon, \epsilon^{\prime}}\right]}$.

Proposition 2.26. The induced maps

$$
\begin{aligned}
& \rho_{\epsilon, \epsilon^{\prime}}: M(\mathbb{K}) \rightarrow \operatorname{Isom}\left(\widehat{\mathbb{K}\left[\mathcal{T}_{\epsilon, \epsilon^{\prime}}\right]}, \widehat{\mathbb{K}\left[\widehat{\mathcal{I} \mathcal{T}_{\epsilon, \epsilon^{\prime}}}\right.}\right),
\end{aligned}
$$

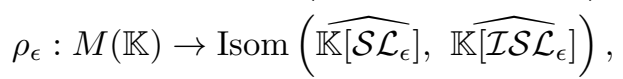

$$
\begin{aligned}
& \rho_{0}: M(\mathbb{K}) \rightarrow \operatorname{Isom}(\widehat{\mathbb{K}[\mathcal{K}]}, \widehat{\mathbb{K}[\mathcal{I} \mathcal{K}]})
\end{aligned}
$$

are all morphisms of bitorsors.

Proof. It is derived from Proposition 1.19,

We may say that the map (2.26) is a generalization of the map (1.19) into proalgebraic tangles.

Next we will discuss a relation of infinitesimal tangles with chord diagrams.

Notation 2.27 (KT, $\mathrm{KRT}$ etc). Let $\Gamma$ be a tangle in $\mathcal{T}_{\epsilon, \epsilon^{\prime}}$. A chord diagram on a curve $\Gamma$ is a finite (possibly empty) set of unordered pair of points on $\Gamma \backslash \partial \Gamma$. A homeomorphism of chord diagrams means a homeomorphism of the underlying curves preserving their orientations and fixing their endpoints such that it preserves the distinguished pairs of points. In our picture, we draw a dashed line, called a chord, between the two points of a distinguished pair.

We denote $\mathcal{C D}_{\epsilon, \epsilon^{\prime}}^{m}$ to be the $\mathbb{K}$-linear space generated by all homeomorphism classes of chord diagrams with $m$ chords $(m \geqslant 0)$ on tangles of type $\left(\epsilon, \epsilon^{\prime}\right)$, subject to the 4T-relation and the FI-relation. Here the $4 T$-relation stands for the 4 terms relation defined by $D_{1}-D_{2}+D_{3}-D_{4}=0$ where $D_{j}$ are chord diagrams with four chords identical outside a ball in which they differ as illustrated in Figure 2.6 and

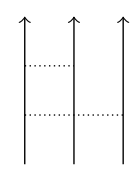

$D_{1}$

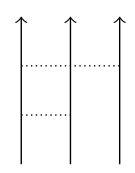

$D_{2}$



$D_{3}$

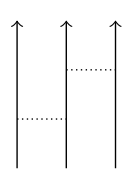

$D_{4}$

FiguRE 2.6. 4T-relation

the FI-relation stands for the frame independent relation where we put $D=0$ for any chord diagrams $D$ with an isolated chord, a chord that does not intersect on any other one in their diagrams. We put $\widehat{\mathcal{C D}}_{\epsilon, \epsilon^{\prime}}:=\widehat{\oplus}_{m=0}^{\infty} \mathcal{C D}_{\epsilon, \epsilon^{\prime}}^{m}$.

It is known that it is well-behaved under the composition

$$
\cdot: \widehat{\mathcal{C D}}_{\epsilon_{3}, \epsilon_{2}} \times \widehat{\mathcal{C D}}_{\epsilon_{2}, \epsilon_{1}} \rightarrow \widehat{\mathcal{C D}}_{\epsilon_{3}, \epsilon_{1}} \text {. }
$$


We denote the subspace of $\widehat{\mathcal{C D}}_{\epsilon, \epsilon^{\prime}}$ consisting of chord diagrams whose underlying spaces are string links with type $\epsilon$ by $\widehat{\mathcal{C D}}(\epsilon)$. It forms a non-commutative $\mathbb{K}$-algebra by the composition map (2.29).

The subspace $\widehat{\mathcal{C D}}(\circlearrowleft)\left(\subset \widehat{\mathcal{C D}}_{\emptyset, \emptyset)}\right.$ of chord diagrams whose underlying spaces are homeomorphic to the oriented circles forms a commutative algebra by the connected sum

$$
\sharp: \widehat{\mathcal{C D}}(\circlearrowleft) \times \widehat{\mathcal{C D}}(\circlearrowleft) \rightarrow \widehat{\mathcal{C D}}(\circlearrowleft)
$$

We remind that the unit is given by the chordless chord diagram on the oriented circle $\bigcirc$.

Proposition 2.28. (1) For each $\epsilon, \epsilon^{\prime}$, there is a natural identifications

$$
\widehat{\mathbb{K}\left[\widehat{\mathcal{I}}_{\epsilon, \epsilon^{\prime}}\right]} \simeq \widehat{\mathcal{C D}}_{\epsilon, \epsilon^{\prime}}
$$

which is compatible with the composition maps.

(2) For each $\epsilon$, there is a natural identification of non-commutative graded $\mathbb{K}$ algebras:



(3) There is a natural identification of commutative graded $\mathbb{K}$-algebras:

$$
\widehat{\mathbb{K}[\mathcal{I K}]} \simeq \widehat{\mathcal{C D}}(\circlearrowleft)
$$

Proof. (1) By replacing each \-part on the associated picture of each infinitesimal tangle $D$ by $~(a c t u a l l y$ we may replace it by because both are equivalent modulo homeomorphisms of underlying tangles) and multiplying $(-1)^{D_{\downarrow}}$ to each $D$ (which is necessary to keep 4T-relation), we obtain a well-defined $\mathbb{K}$-linear map

$$
\widehat{\mathbb{K}\left[\widehat{\mathcal{I T}}_{\epsilon, \epsilon^{\prime}}\right]} \rightarrow \widehat{\mathcal{C D}}_{\epsilon, \epsilon^{\prime}} \text {. }
$$

Here $D_{\downarrow}$ is the the set of ends of chord on $D$ which hit downward lines. Its composition with the map $\rho_{\epsilon, \epsilon^{\prime}}(p)$ in (2.22) is the isomorphism

$$
\widehat{\mathbb{K}\left[\widehat{\mathcal{T}}_{\epsilon, \epsilon^{\prime}}\right]} \rightarrow \widehat{\mathcal{C D}}_{\epsilon, \epsilon^{\prime}}
$$

given as the non-framed version of $[\mathrm{KT}]$ Theorem 2.4. Since $\rho_{\epsilon, \epsilon^{\prime}}(p)$ is isomorphic, the morphism in our claim should be isomorphic. Checking the compatibility with the composition map is immediate.

(2) It immediately follows from (1).

(3) It is obtained by a restriction of the above claim into the case $\left(\epsilon, \epsilon^{\prime}\right)=(\emptyset, \emptyset)$. It can be checked directly that the map is compatible with the connected sum.

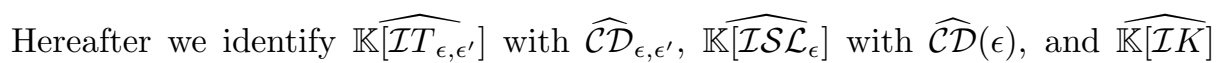
with $\widehat{\mathcal{C D}}(\bigcirc)$ by the map (2.31). Thus the identification given in Lemma 2.21 is reformulated as the identification below

$$
(\widehat{\mathcal{C D}}(\epsilon), \cdot) \simeq(\widehat{\mathcal{C D}}(\circlearrowleft), \sharp)
$$

for $\epsilon=\uparrow$ or $\downarrow$.

Remark 2.29. Kontsevich's isomorphism ( $[\mathrm{K}]$ )

$$
I: \widehat{\mathbb{C}[\mathcal{K}]} \simeq \widehat{\mathcal{C D}}(\circlearrowleft)
$$


is given by specifying $p$ of $\rho_{0}(p)$ of (2.24) to $p_{\mathrm{KZ}}=\left(1, \varphi_{\mathrm{KZ}}\right) \in M(\mathbb{C})$ with $\varphi_{\mathrm{KZ}}=$ $\Phi_{\mathrm{KZ}}\left(\frac{1}{2 \pi \sqrt{-1}} A, \frac{1}{2 \pi \sqrt{-1}} B\right.$ ). We note that it is independent of the choice of $\varphi$ (cf. [LM1]). For each oriented knot $K$, the image $I(K)$ is called the Kontsevich invariant of $K$.

Remark 2.30. By the same arguments to Remark 1.23, the proalgebraic tangles $\widehat{\mathbb{Q}\left[\mathcal{T}_{\epsilon, \epsilon^{\prime}}\right]}$, the proalgebraic string links $\widehat{\mathbb{Q}\left[\mathcal{S L}_{\epsilon}\right]}$ and the proalgebraic knots $\widehat{\mathbb{K}[\mathcal{K}]}$ are regarded as Betti realizations of mixed Tate (pro-)motives over Spec $\mathbb{Z}$. And their corresponding de Rham realizations are given by the spaces $\widehat{\mathcal{C D}}_{\epsilon, \epsilon^{\prime}}, \widehat{\mathcal{C D}}(\epsilon)$ and $\widehat{\mathcal{C D}}(\circlearrowleft)$ of chord diagrams located there respectively. In Remark 3.28 , we will see that the proalgebraic knots $\widehat{\mathbb{K}[\mathcal{K}]}$ carries a structure of Tate (pro-)motives.

\section{Main Results}

We discuss and derive distinguished properties of the action of the GrothendieckTeichmüller groups on proalgebraic tangles (constructed in 92.1 ) which can not be observed in the action on proalgebraic braids (discussed in \$1.1). By exploiting the properties, we explicitly determine the proalgebraic knot whose Kontsevich invariant is the unit, the trivial chord diagram (Theorem 3.22).

3.1. Proalgebraic string links. We restrict the previously constructed action of the Grothendieck-Teichmüller group $G T(\mathbb{K})$ on proalgebraic tangles into the action of its unipotent part $G T_{1}(\mathbb{K})$ on proalgebraic string links and show that it is simply described by an inner conjugation (Proposition 3.10 and Theorem 3.14). The proofs are based on Twistor Lemmas (Lemma 3.2 and 3.12).

Notation 3.1. (1) For $n>1, \epsilon_{i}: \widetilde{\mathbb{K}\left[\widehat{\mathcal{I S \mathcal { L }}} \uparrow^{n}\right]} \rightarrow \mathbb{K}\left[\widehat{\mathcal{I} \mathcal{S L}_{\uparrow n-1}}\right](i=1,2 \ldots n)$ means the map sending an infinitesimal string link $D$ to 0 if at least one chord of $D$ has an endpoint on the $i$-th strand; otherwise $\epsilon_{i}(D)$ is obtained by removing the $i$-th strand.

(2) For $D \in \mathbb{\mathbb { K }} \widehat{\left.\mathcal{I S \mathcal { L }}_{\uparrow n}\right]}$, we denote $D_{1, \ldots, n}$ (resp. $\left.D_{2, \ldots, n+1}\right)$ to be the element in $\mathbb{K}\left[\widehat{\mathcal{I} \mathcal{S} \mathcal{L}_{\uparrow n+1}}\right]$ obtained by putting a chordless straight line on the right (resp. the left) of $D$ and $D_{1, \ldots, i-1, i} i+1, i+2, \ldots, n+1(i=1,2, \ldots, n)$ to be also the element in $\mathbb{K}\left[\widehat{\mathcal{I S \mathcal { L L } _ { \uparrow n + 1 }}}\right]$ obtained by doubling the $i$-th strand and taking the sum over all possible lifts of the chord endpoints of $D$ from the $i$-th strand to one of the new two strands.

(3) For $D \in \mathbb{K}\left[\widehat{\mathcal{I} \mathcal{S L}_{\uparrow n}}\right]$ and $\tau \in \mathfrak{S}_{n}, D_{\tau(1), \ldots, \tau(n)}$ denote the element in $\tau^{-1}$. $D \cdot \tau$ where the product is taken as a product of infinitesimal tangles (recall the identification given in Proposition 2.28).

Hereafter we regard $\exp \mathfrak{f}_{2}$ and $U \mathfrak{p}_{5}$ to be the subspaces of $\mathbb{K}[\widehat{\mathcal{I S \mathcal { L }} \uparrow \uparrow \uparrow}]$ and $\mathbb{K}\left[\widehat{\mathcal{I S \mathcal { L } _ { \uparrow \uparrow \uparrow \uparrow }}}\right]$ respectively. The following lemma which is shown for $G R T(\mathbb{K})$ might be called as a reformulation of [LM1] Theorem 8 which is shown for a 'chord diagrammatic' analogue of $M_{1}(\mathbb{K})$.

Lemma 3.2 (Twistor Lemma). Let $\sigma=(c, g) \in G R T(\mathbb{K})$, thus $c \in \mathbb{K}^{\times}$and $g \in \exp \mathfrak{f}_{2} \subset \mathbb{K}[\widehat{\mathcal{I S \mathcal { L }}} \uparrow \uparrow \uparrow]$. Then $g$ is gauge equivalent to 1 , namely, there exists

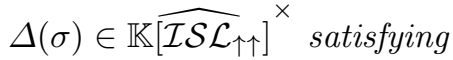

$$
\epsilon_{1}(\Delta(\sigma))=\epsilon_{2}(\Delta(\sigma))=\uparrow
$$


and the symmetric condition

$$
\Delta(\sigma)=\Delta(\sigma)_{2,1}
$$

such that

$$
g=\Delta(\sigma)_{2,3} \cdot \Delta(\sigma)_{1,23} \cdot \Delta(\sigma)_{12,3}^{-1} \cdot \Delta(\sigma)_{1,2}^{-1}
$$

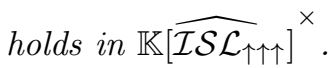

Proof. The proof can be done recursively in the same way to the proof of [LM1] Theorem 8, or rather, we may say that actually it is easier: Because we have $(1, g)$ and $(1,1) \in G R T_{1}(\mathbb{K})$, both $g$ and 1 satisfy the same relations (1.8)-(1.10). Our proof is obtained just by replacing $\Phi$ by $g$ and $\Phi^{\prime}$ by 1 in their proof.

Remark 3.3. Other variants of twistor lemma can be found in several literatures such as [LS] Theorem 2.1 with twistors in Aut $\widehat{F}_{2}$ for $\widehat{G T}_{1}$, AT2 Theorem 7.5 with twistors in $\mathrm{TAutf}_{2}$ for $K R V_{3}^{0}$, and [AET] Theorem 2 with twistors in TAutf $f_{2}$ for $M_{1}(\mathbb{K})$. Actually all of them are attributed to $[\mathrm{Dr}$ Theorem A'.

The above $\Delta(\sigma)$ may not be uniquely chosen but it can be chosen independently from $c$ by the construction. When we make such a choice, we occasionally denote $\Delta(g)$ instead of $\Delta(\sigma)$ by abuse of notations. We note that the first two terms on the right hand side of (3.3) commute each other, so do the last two terms.

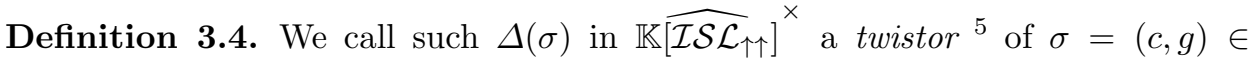
$G R T(\mathbb{K})$. For a twisor $\Delta(\sigma)$, we put $\Delta(\sigma, \uparrow):=\uparrow \in \mathbb{K} \widehat{\mathcal{I S \mathcal { L }}} \uparrow_{\uparrow}{ }^{\times}$and

$$
\Delta\left(\sigma, \uparrow^{n}\right):=\Delta(\sigma)_{12 \cdots n-1, n} \cdots \Delta(\sigma)_{12,3} \cdot \Delta(\sigma)_{1,2} \in \mathbb{K}_{\left.\mathcal{I \mathcal { S L }}_{\uparrow n}\right]} \times
$$

for $n \geqslant 2$. Here $\Delta(\sigma)_{1 \cdots k, k+1}$ means the element in $\left.\mathbb{\mathbb { K }} \widehat{\mathcal{I S \mathcal { L }}_{\uparrow n}}\right]$ obtained multidoubling of the first strand of $\Delta(\sigma) \in \mathbb{\mathbb { K }} \widehat{\left[\mathcal{I S L}_{\uparrow \uparrow}\right]}$ by $k$ strands, summing up all possible lifts of chords and putting $n-k-1$ chordless line $\uparrow^{n-k-1}$ on its right.

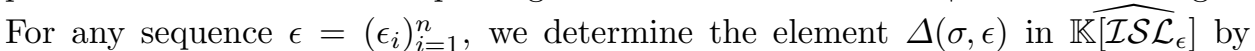
reversing corresponding all arrows of $\Delta\left(\sigma, \uparrow^{n}\right)$. We note that $\Delta(\sigma, \uparrow \uparrow)=\Delta(\sigma)$ and $g=\Delta(\sigma, \uparrow \uparrow \uparrow)_{3,2,1} \cdot \Delta(\sigma, \uparrow \uparrow \uparrow)^{-1}$ by (3.2).

An automorphism $\theta_{\epsilon, \epsilon^{\prime}}^{\Delta(\sigma)}$ of $\mathbb{K} \widehat{\left[\mathcal{I T}_{\epsilon, \epsilon^{\prime}}\right]}$ associated to a twistor $\Delta(\sigma)$ can be constructed piecewise as follows.

(1) when $D \in A_{k, l}^{\epsilon}$, we define

$$
\theta_{\epsilon, \epsilon^{\prime}}^{\Delta(\sigma)}(D):=D \cdot\left(\Delta(\sigma)_{k+1, k+2}^{-1}\right)^{s(D)} \cdot\left(\nu_{g}\right)_{k+2}^{s(D)} \cdot g_{1 \cdots k, k+1, k+2}^{s(D)} .
$$

Here the term $\left(\Delta(\sigma)_{k+1, k+2}^{-1}\right)^{s(D)}$ means the element in $\left.\widehat{\mathbb{K}[\widehat{\mathcal{I S \mathcal { L }}}(D)}\right]$ obtained by putting the trivial chordless diagram with $k$-strands on the left of $\Delta(\sigma)^{-1}$ and and the trivial chordless diagram with $l$-strands on its right.

(2) when $D=\left(b_{n}, \epsilon\right) \in \widehat{I B_{\tau}^{\epsilon}}$, we define

$$
\theta_{\epsilon, \epsilon^{\prime}}^{\Delta(\sigma)}(D):=\left(\rho_{n}(\sigma)\left(b_{n}\right), \epsilon\right)
$$

(3) when $D \in C_{k, l}^{\epsilon}$, we define

$$
\theta_{\epsilon, \epsilon^{\prime}}^{\Delta(\sigma)}(D):=g_{1 \cdots k, k+1, k+2}^{-1, t(D)} \cdot\left(\Delta(\sigma)_{k+1, k+2}\right)^{s(D)} \cdot D .
$$

\footnotetext{
${ }^{5}$ We call this element twistor because it is related to Drinfeld's notion of twisting in $\mathrm{Dr}$.
} 
Here $\mu_{g}^{\epsilon}$ is the one defined in Definition 2.22 .

Lemma 3.5. For any sequences $\epsilon, \epsilon^{\prime}$, any $\sigma \in G R T(\mathbb{K})$ and any twistor $\Delta(\sigma)$, the above construction determines a well-defined automorphism $\theta_{\epsilon, \epsilon^{\prime}}^{\Delta(\sigma)}$ of $\left.\overline{\mathbb{K}} \widehat{\mathcal{I} \mathcal{T}_{\epsilon, \epsilon^{\prime}}}\right]$ which is compatible with the composition map (2.29).

Proof. This can be verified by an almost same way to the proof of Proposition 2.23 except for compatibilities of (IT5) and (IT6).

- To check the compatibility of the first equality of (IT5), it is enough to show the equality illustrated in Figure 3.1. By the identification of $\overline{\mathbb{K}\left[\mathcal{I S L}_{\uparrow}\right]}$ with $\widehat{\mathbb{K}[\mathcal{I K}]}$ given in Lemma 2.21 (cf. $(2.32)$ ), showing the validity is deduced to showing the equality illustrated in Figure 3.2. It is immediate to see because we have (IT6) and $\Delta(\sigma)_{2,1}=\Delta(\sigma)$ by (3.2).

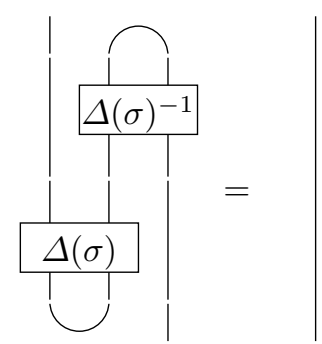

FiguRE 3.1

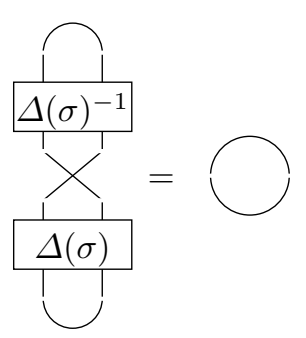

FiguRE 3.2

The compatibility of the second equality of (IT5) can be done in the same way.

- To check the compatibility of the first equality of (IT6), it is enough to show the equality illustrated in Figure 3.3 Actually it is a consequence of (IT3), (IT5) and (IT6). The compatibility of the second equality of (IT6) can be done in the same way.

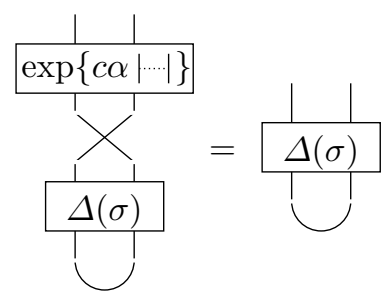

FIGURE 3.3

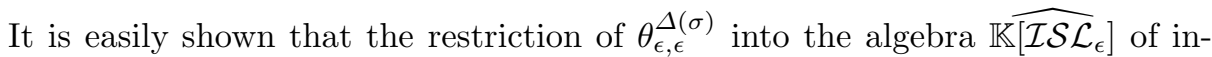
finitesimal string links induces its automorphism, denoted by $\theta_{\epsilon}^{\Delta(\sigma)}$. The following explains its relationship with our previous automorphism $\rho_{\epsilon}(\sigma)$ in (2.18). 
Proposition 3.6. For any $\sigma \in G R T(\mathbb{K})$ and any sequence $\epsilon$,

$$
\rho_{\epsilon}(\sigma)(D)=\theta_{\epsilon}^{\Delta(\sigma)}(D)
$$

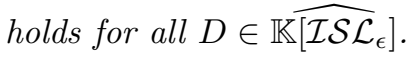

Proof. Differences between two actions $\theta_{\epsilon}^{\Delta(\sigma)}$ and $\rho_{\epsilon}(\sigma)$ are observed only on the action on $A_{k, l}^{\epsilon}$ and $C_{k, l}^{\epsilon}$. It is enough to show that equality of Figure 3.1 which were proved in the above lemma.

When we restrict $\sigma$ into the unipotent part $G R T_{1}(\mathbb{K})$, we obtain a relationship of $\theta_{\epsilon, \epsilon^{\prime}}^{\Delta(\sigma)}$ with the identity map of $\widehat{\mathbb{K}} \widehat{\left.\mathcal{I T}_{\epsilon, \epsilon^{\prime}}\right]}$ as follows.

Proposition 3.7. For any $\sigma=(1, g) \in G R T_{1}(\mathbb{K})$ and any sequences $\epsilon$ and $\epsilon^{\prime}$,

$$
\theta_{\epsilon, \epsilon^{\prime}}^{\Delta(\sigma)}(D)=\Delta(\sigma, \epsilon) \cdot D \cdot \Delta\left(\sigma, \epsilon^{\prime}\right)^{-1} .
$$

holds for any $\left.D \in \widehat{\mathbb{K}} \widehat{\left[\mathcal{I T}_{\epsilon, \epsilon^{\prime}}\right.}\right]$.

Proof. The equation can be checked piecewise.

- When $D \in A_{k, l}^{\epsilon}$, we may assume that $D=a_{k, l}^{\epsilon}$ with $s(D)=\epsilon_{1}$ and $t(D)=$ $\epsilon_{2}$. Then

$$
\begin{aligned}
\theta_{\epsilon_{2}, \epsilon_{1}}^{\Delta(\sigma)}\left(a_{k, l}^{\epsilon}\right)= & a_{k, l}^{\epsilon} \cdot \Delta(\sigma)_{k+1, k+2}^{-1} \cdot\left(\mu_{g}\right)_{k+2} \cdot g_{1 \cdots k, k+1, k+2} \\
= & a_{k, l}^{\epsilon} \cdot \Delta(\sigma)_{k+1, k+2}^{-1} \cdot\left(\mu_{g}\right)_{k+2} \\
& \cdot \Delta(\sigma)_{k+1, k+2} \cdot \Delta(\sigma)_{1 \cdots k, k+1} k+2 \cdot \Delta(\sigma)_{1 \cdots k+1, k+2}^{-1} \cdot \Delta(\sigma)_{1 \cdots k, k+1}^{-1} \\
= & a_{k, l}^{\epsilon} \cdot\left(\mu_{g}\right)_{k+2} \cdot \Delta(\sigma)_{1 \cdots k+1, k+2}^{-1} \cdot \Delta(\sigma)_{1 \cdots k, k+1}^{-1} .
\end{aligned}
$$

By Lemma 3.8 ,

$$
\begin{aligned}
& =a_{k, l}^{\epsilon} \cdot \Delta(\sigma)_{1 \cdots k+1, k+2}^{-1} \cdot \Delta(\sigma)_{1 \cdots k, k+1}^{-1} \\
& =\Delta(\sigma)_{1,2} \cdots \Delta(\sigma)_{1 \cdots k+l-1, k+l} \cdot a_{k, l}^{\epsilon} \cdot \Delta(\sigma)_{1 \cdots k+l+1, k+l+2}^{-1} \cdots \Delta(\sigma)_{1,2}^{-1} \\
& =\Delta\left(\sigma, \epsilon_{2}\right) \cdot a_{k, l}^{\epsilon} \cdot \Delta\left(\sigma, \epsilon_{1}\right)^{-1} .
\end{aligned}
$$

- When $D \in \widehat{I B}$, it is enough to to show the case when $D=\tau_{i, i+1}$ or $t_{i, i+1} \in U \mathfrak{b}_{n}(1 \leqslant i \leqslant n-1)$. If $D=\tau_{i, i+1}$ with $s(D)=\epsilon_{1}$ and $t(D)=\epsilon_{2}$, by Proposition 1.12 ,

$$
\begin{aligned}
\theta_{\epsilon_{2}, \epsilon_{1}}^{\Delta(\sigma)}\left(\tau_{i, i+1}\right)= & g_{1 \cdots i-1, i, i+1}^{-1} \cdot \tau_{i, i+1} \cdot g_{1 \cdots i-1, i, i+1} \\
= & \Delta(\sigma)_{1 \cdots i-1, i} \cdot \Delta(\sigma)_{1 \cdots i, i+1} \cdot \Delta(\sigma)_{1 \cdots i-1, i i+1}^{-1} \cdot \Delta(\sigma)_{i, i+1}^{-1} \\
& \cdot \tau_{i, i+1} \cdot \Delta(\sigma)_{i, i+1} \cdot \Delta(\sigma)_{1 \cdots i-1, i} i_{i+1} \cdot \Delta(\sigma)_{1 \cdots i, i+1}^{-1} \cdot \Delta(\sigma)_{1 \cdots i-1, i}^{-1} .
\end{aligned}
$$

By (3.2),

$$
\begin{aligned}
& =\Delta(\sigma)_{1 \cdots i-1, i} \cdot \Delta(\sigma)_{1 \cdots i, i+1} \cdot \tau_{i, i+1} \cdot \Delta(\sigma)_{1 \cdots i, i+1}^{-1} \cdot \Delta(\sigma)_{1 \cdots i-1, i}^{-1} \\
& =\Delta(\sigma)_{1,2} \cdots \Delta(\sigma)_{1 \cdots n-1, n} \cdot \tau_{i, i+1} \cdot \Delta(\sigma)_{1 \cdots n-1, n}^{-1} \cdots \Delta(\sigma)_{1,2}^{-1} \\
& =\Delta\left(\sigma, \epsilon_{2}\right) \cdot \tau_{i, i+1} \cdot \Delta\left(\sigma, \epsilon_{1}\right)^{-1}
\end{aligned}
$$


If $D=t_{i, i+1}$ with $s(D)=\epsilon_{1}$ and $t(D)=\epsilon_{2}$, again by Proposition 1.12,

$$
\begin{aligned}
\theta_{\epsilon_{2}, \epsilon_{1}}^{\Delta(\sigma)}\left(t_{i, i+1}\right)= & g_{1 \cdots i-1, i, i+1}^{-1} \cdot t_{i, i+1} \cdot g_{1 \cdots i-1, i, i+1} \\
= & \Delta(\sigma)_{1 \cdots i-1, i} \cdot \Delta(\sigma)_{1 \cdots i, i+1} \cdot \Delta(\sigma)_{1 \cdots i-1, i}^{-1} i+1 \cdot \Delta(\sigma)_{i, i+1}^{-1} \\
& \cdot t_{i, i+1} \cdot \Delta(\sigma)_{i, i+1} \cdot \Delta(\sigma)_{1 \cdots i-1, i} i_{i+1} \cdot \Delta(\sigma)_{1 \cdots i, i+1}^{-1} \cdot \Delta(\sigma)_{1 \cdots i-1, i}^{-1}
\end{aligned}
$$

By Lemma 3.9,

$$
\begin{aligned}
& =\Delta(\sigma)_{1 \cdots i-1, i} \cdot \Delta(\sigma)_{1 \cdots i, i+1} \cdot t_{i, i+1} \cdot \Delta(\sigma)_{1 \cdots i, i+1}^{-1} \cdot \Delta(\sigma)_{1 \cdots i-1, i}^{-1} \\
& =\Delta(\sigma)_{1,2} \cdots \Delta(\sigma)_{1 \cdots n-1, n} \cdot t_{i, i+1} \cdot \Delta(\sigma)_{1 \cdots n-1, n}^{-1} \cdots \Delta(\sigma)_{1,2}^{-1} \\
& =\Delta\left(\sigma, \epsilon_{2}\right) \cdot t_{i, i+1} \cdot \Delta\left(\sigma, \epsilon_{1}\right)^{-1} .
\end{aligned}
$$

We note that we use $c=1$ for the second equality.

- When $D \in C_{k, l}^{\epsilon}$, we may assume that $D=c_{k, l}^{\epsilon}$ with $s(D)=\epsilon_{1}$ and $t(D)=$ $\epsilon_{2}$. Then

$$
\begin{aligned}
\theta_{\epsilon_{2}, \epsilon_{1}}^{\Delta(\sigma)}\left(c_{k, l}^{\epsilon}\right)= & g_{1 \cdots k, k+1, k+2}^{-1} \cdot \Delta(\sigma)_{k+1, k+2} \cdot c_{k, l}^{\epsilon} \\
= & \Delta(\sigma)_{1 \cdots k, k+1} \cdot \Delta(\sigma)_{1 \cdots k+1, k+2} \cdot \Delta(\sigma)_{1 \cdots k, k+1}^{-1} k+2 \cdot \Delta(\sigma)_{k+1, k+2}^{-1} \\
& \cdot \Delta(\sigma)_{k+1, k+2} \cdot c_{k, l}^{\epsilon} \\
= & \Delta(\sigma)_{1 \cdots k, k+1} \cdot \Delta(\sigma)_{1 \cdots k+1, k+2} \cdot c_{k, l}^{\epsilon} \\
= & \Delta(\sigma)_{1,2} \cdots \Delta(\sigma)_{1 \cdots k+l+1, k+l+2} \cdot c_{k, l}^{\epsilon} \cdot \Delta(\sigma)_{1 \cdots k+l, k+l}^{-1} \cdots \Delta(\sigma)_{1,2}^{-1} \\
= & \Delta\left(\sigma, \epsilon_{2}\right) \cdot c_{k, l}^{\epsilon} \cdot \Delta\left(\sigma, \epsilon_{1}\right)^{-1} .
\end{aligned}
$$

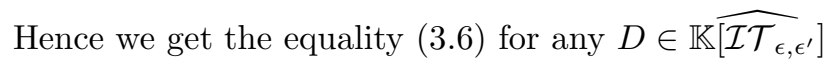

The followings are required to prove the previous proposition.

Lemma 3.8. For $\sigma=(c, g) \in G R T(\mathbb{K})$, the infinitesimal long knot $\mu_{g} \in \widehat{\mathbb{K}\left[\widehat{\mathcal{I S L}}_{\uparrow}\right]}$ is actually equal to the trivial chordless chord diagram on $\uparrow$.

Proof. It is enough to show that $\Lambda_{g}$ is the trivial diagram $\downarrow$. Since $g=\Delta(\sigma)_{2,3}$. $\Delta(\sigma)_{1,23} \cdot \Delta(\sigma)_{12,3}^{-1} \cdot \Delta(\sigma)_{1,2}^{-1}$, it is enough to show the equality depicted in Figure 3.4. It can be proved in a same way to Figure 3.2 .

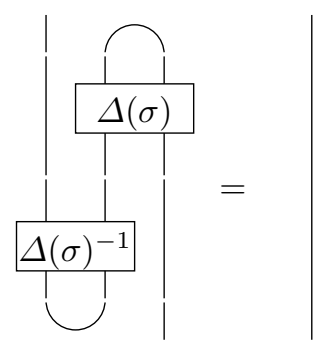

Figure 3.4. Proof of Lemma 3.8

The following is proved in a topological way. 




$$
t_{12} \cdot D=D \cdot t_{12}
$$

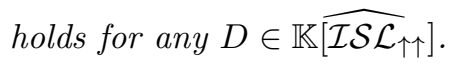

Proof. First, for a discrete case, we have $T \cdot\left(\sigma_{1,2}\right)^{2 \alpha}=\left(\sigma_{1,2}\right)^{2 \alpha} \cdot T$ for any $T \in \mathcal{S L}(\uparrow \uparrow)$ and $\alpha \in \mathbb{Z}$. Whence we will have the same equality $T \cdot\left(\sigma_{1,2}\right)^{2 \alpha}=\left(\sigma_{1,2}\right)^{2 \alpha} \cdot T$ for any $T \in \widehat{\mathbb{K}\left[\widehat{\mathcal{S} \mathcal{L}_{\uparrow \uparrow}}\right]}$ and $\alpha \in \mathbb{K}$. Then by the isomorphism (2.23), we have $D \cdot \exp \left\{\alpha t_{1,2}\right\}=\exp \left\{\alpha t_{1,2}\right\} \cdot D$ for any $D \in \mathbb{\mathbb { K } [ \widehat { \mathcal { I S L } } _ { \uparrow \uparrow } ]}$ and $\alpha \in \mathbb{K}$. Since the space

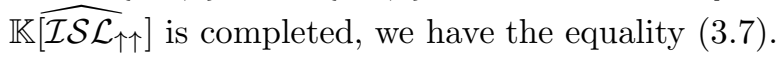

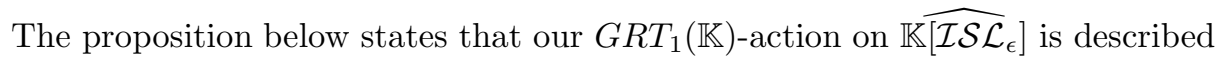
by an inner conjugation action of twistor.

Proposition 3.10. Let $\epsilon$ be any sequence. The action $\rho_{\epsilon}$ of (2.19) restricted into

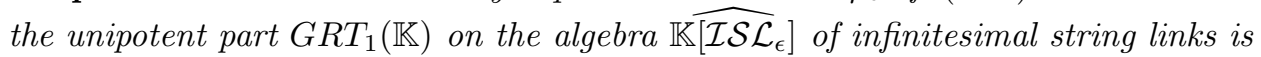
given by the inner conjugation of the twistor $\Delta(\sigma, \epsilon)$, i.e.

$$
\rho_{\epsilon}(\sigma)(D)=\Delta(\sigma, \epsilon) \cdot D \cdot \Delta(\sigma, \epsilon)^{-1}
$$

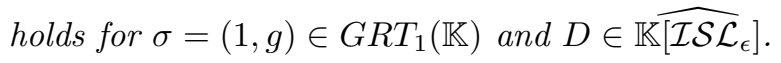

Proof. The formula is obtained by combining (3.6) and (3.7).

Next we discuss $G T_{1}(\mathbb{K})$-action on the algebra $\widehat{\mathbb{K}\left[\mathcal{S L}_{\epsilon}\right]}$ of proalgebraic string links.


the map removing the $i$-th strand on each proalgebraic string links.

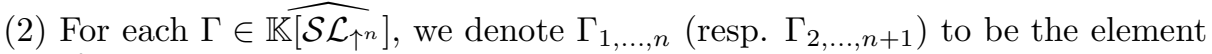
in $\mathbb{K}\left[\widehat{\mathcal{S} \mathcal{L}_{\uparrow n+1}}\right]$ obtained by putting a straight line on the right (resp. the left) of $\Gamma$ and $\left.\Gamma_{1, \ldots, i-1, i} i+1, i+2, \ldots, n+1=1,2, \ldots, n\right)$ to be also the element in $\mathbb{K}\left[\widehat{\mathcal{S} \mathcal{L}_{\uparrow n+1}}\right]$ obtained by doubling the $i$-th strand.

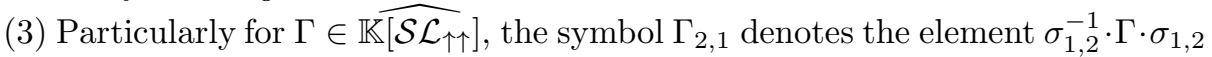

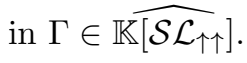

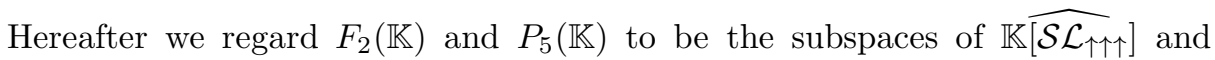
$\mathbb{K}\left[\widehat{\mathcal{S} \mathcal{L}_{\uparrow \uparrow \uparrow \uparrow}}\right]$ respectively. The following lemma is a $G T(\mathbb{K})$-analogue of Twistor Lemma 3.2 .

Lemma 3.12 (Twistor Lemma). Let $\sigma=(\lambda, f) \in G T(\mathbb{K})$, thus $\lambda \in \mathbb{K}^{\times}$and $f \in F_{2}(\mathbb{K}) \subset \widehat{\mathbb{K}} \widehat{\left[\mathcal{S L}_{\uparrow \uparrow \uparrow}\right]}$. Then $f$ is gauge equivalent to 1 , namely, there exists $\varpi(\sigma) \in{\widehat{\mathbb{K}\left[\mathcal{S L}_{\uparrow \uparrow}\right]}}^{\times}$satisfying

$$
\epsilon_{1}(\varpi(\sigma))=\epsilon_{2}(\varpi(\sigma))=\uparrow
$$

and the symmetric condition

$$
\varpi(\sigma)=\varpi(\sigma)_{2,1}
$$

such that

$$
f=\varpi(\sigma)_{2,3} \cdot \varpi(\sigma)_{1,23} \cdot \varpi(\sigma)_{12,3}^{-1} \cdot \varpi(\sigma)_{1,2}^{-1}
$$


holds in $\widehat{\mathbb{K}[\widehat{\mathcal{S L}} \uparrow \uparrow \uparrow]^{\times}}$.

Proof. Fix an element $p=(1, \varphi) \in M_{1}(\mathbb{K})$. Then by Proposition 1.18 it yields an isomorphism

$$
r_{p}: G T_{1}(\mathbb{K}) \simeq G R T_{1}(\mathbb{K}) .
$$

By Proposition 2.25. (3), we have an isomorphism

$$
\left.\rho_{\uparrow \uparrow \uparrow}(p): \widetilde{\mathbb{K}} \widehat{\left[\mathcal{S L}_{\uparrow \uparrow \uparrow}\right.}\right] \simeq \mathbb{K}\left[\widehat{\mathcal{I} \mathcal{S} \mathcal{L}_{\uparrow \uparrow \uparrow}}\right] .
$$

Put $\varpi(\sigma):=\rho_{\uparrow \uparrow \uparrow}(p)^{-1}\left(\Delta\left(r_{p}(\sigma)\right)\right)$. Then using Proposition 2.26 and Twistor Lemma 3.2, we can check the validities of (3.9) - (3.11) for $\varpi(\sigma)$ by direct calculations.

The above $\varpi(\sigma)$ may not be uniquely chosen and may depend on $\lambda \in \mathbb{K}^{\times}$unlike the case for $G R T(\mathbb{K})$. Here we note again that the first two terms on the right hand side of (3.3) commute each other, so do the last two terms.

Definition 3.13. We call such $\varpi(\sigma)$ in ${\widehat{\mathbb{K}\left[\mathcal{S L}_{\uparrow \uparrow}\right]}}^{\times}$a twistor of $\sigma=(\lambda, f) \in G T(\mathbb{K})$. For a twisor $\varpi(\sigma)$, we put $\varpi(\sigma, \uparrow):=\uparrow \in{\widehat{\mathbb{K}\left[\mathcal{S L}_{\uparrow}\right.}}^{\times}$and

$$
\varpi(\sigma, \uparrow n):=\varpi(\sigma)_{12 \cdots n-1, n} \cdots \varpi(\sigma)_{12,3} \cdot \varpi(\sigma)_{1,2} \in{\widehat{\mathbb{K}\left[\mathcal{S L}_{\uparrow n}\right]}}^{\times}
$$

for $n \geqslant 2$. Here $\varpi(\sigma)_{1 \cdots k, k+1}$ means the element in $\widehat{\mathbb{K}\left[\mathcal{S L}_{\uparrow n}\right]}$ obtained multi-


straight lines $\uparrow^{n-k-1}$ on its right. For any sequence $\epsilon=\left(\epsilon_{i}\right)_{i=1}^{n}$, we determine the element $\varpi(\sigma, \epsilon)$ in ${\widehat{\mathbb{K}\left[\mathcal{S L}_{\epsilon}\right]}}^{\times}$by reversing all corresponding arrows of $\varpi(\sigma, \uparrow n)$. We note that $\varpi(\sigma, \uparrow \uparrow)=\varpi(\sigma)$ and $f=\varpi(\sigma, \uparrow \uparrow \uparrow)_{3,2,1} \cdot \varpi(\sigma, \uparrow \uparrow \uparrow)^{-1}$ by (3.10).

Our theorem in this subsection is to state that our $G T_{1}(\mathbb{K})$-action on $\widehat{\mathbb{K}\left[\mathcal{S} \mathcal{L}_{\epsilon}\right]}$ is described by the inner conjugation action by the twistor.

Theorem 3.14. Let $\epsilon$ be any sequence. The action

$$
\rho_{\epsilon}: G T_{1}(\mathbb{K}) \rightarrow \text { Aut } \widehat{\mathbb{K}\left[\mathcal{S L}_{\epsilon}\right]}
$$

induced by (2.10), of the unipotent part $G T_{1}(\mathbb{K})$ on the algebra $\widehat{\mathbb{K}\left[\mathcal{S L}_{\epsilon}\right]}$ of proalgebraic string links is simply given by an inner conjugation of twistor $\varpi(\sigma, \epsilon) \in$ $\widehat{\mathbb{K}\left[\mathcal{S L}_{\epsilon}\right]} \times$. That is,

$$
\rho_{\epsilon}(\sigma)(\Gamma)=\varpi(\sigma, \epsilon) \cdot \Gamma \cdot \varpi(\sigma, \epsilon)^{-1}
$$

holds for $\sigma=(1, f) \in G T_{1}(\mathbb{K})$ and $\Gamma \in \widehat{\mathbb{K}\left[\mathcal{S L}_{\epsilon}\right]}$.

Proof. Let $p=(1, \varphi) \in M_{1}(\mathbb{K})$ be an element taken in the proof of Twistor Lemma 3.12. By Proposition 1.18 it yields an isomorphism $r_{p}: G T_{1}(\mathbb{K}) \simeq G R T_{1}(\mathbb{K})$. By Proposition 2.25.(3), we have an isomorphism

$$
\rho_{\epsilon}(p): \widehat{\mathbb{K}\left[\widehat{\mathcal{S L}} \mathcal{L}_{\uparrow n}\right]} \simeq \widetilde{\mathbb{K}[\widehat{\mathcal{I S \mathcal { L }}} \uparrow n} .
$$

Our claim follows from Proposition 2.26 and 3.10. We may take $\varpi(\sigma, \epsilon)$ in the above (3.13). by $\rho_{\epsilon}(p)^{-1}\left(\Delta\left(r_{p}(\sigma), \epsilon\right)\right)$.

Here is a corollary of Theorem 3.14 
Corollary 3.15. The restricted action of (1.5) into the unipotent part $G T_{1}(\mathbb{K})$ on proalgebraic pure braids, denoted by

$$
\rho_{n}: G T_{1}(\mathbb{K}) \rightarrow \text { Aut } \widehat{\mathbb{K}\left[P_{n}\right]},
$$


is,

$$
\rho_{n}(\sigma)\left(x_{i j}\right)=\varpi\left(\sigma, \uparrow^{n}\right) \cdot x_{i j} \cdot \varpi\left(\sigma, \uparrow^{n}\right)^{-1}
$$

holds for $1 \leqslant i, j \leqslant n$ and $\sigma \in G T_{1}(\mathbb{K})$.

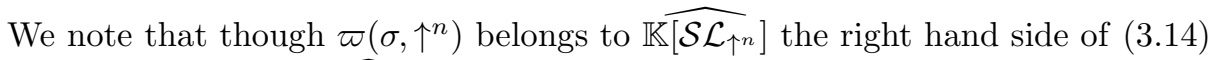
belongs to its subspace $\widehat{\mathbb{K}\left[P_{n}\right]}$.

Remark 3.16. (1) Since the $G T(\mathbb{K})$-action on $\widehat{\mathbb{K}\left[P_{n}\right]}$ is faithful for $n \geqslant 3$, the action $\rho_{\epsilon}: G T_{1}(\mathbb{K}) \rightarrow$ Aut $\widehat{\mathbb{K}\left[\mathcal{S \mathcal { L } _ { \epsilon }}\right]}$ is faithful for $n \geqslant 3$, where $n$ is the number of strings, i.e. the cardinality of $\epsilon$, by Remark 2.8 .

(2) When $n=1$, the action is far from faithful. Actually the kernel of the action $\rho_{\epsilon}: G T_{1}(\mathbb{K}) \rightarrow$ Aut $\widehat{\mathbb{K}\left[\mathcal{S L}_{\epsilon}\right]}$ (with $\epsilon=\uparrow$ or $\downarrow$ ) on proalgebraic long knots is the unipotent part $G T_{1}(\mathbb{K})$ because $\varpi(\sigma, \epsilon)$ in Theorem 3.14 is trivial.

The rest case $n=2$ looks unclear.

Problem 3.17. Is the action

$$
\rho_{\epsilon}: G T(\mathbb{K}) \rightarrow \text { Aut } \widehat{\mathbb{K}\left[\widehat{\mathcal{S}} \mathcal{L}_{\epsilon}\right]}
$$

(with $\epsilon=\left(\epsilon_{1}, \epsilon_{2}\right) \in\{\uparrow, \downarrow\}^{2}$ ) on proalgebraic 2-string links faithful?

3.2. Proalgebraic knots. We discuss a non-trivial grading on proalgebraic knots induced by $G T(\mathbb{K})$-action. In $[\mathrm{BLT}]$ the image of the trivial knot, the unknot, under the Kontsevich isomorphism (2.33) is explicitly determined. In this subsection, we work in an opposite direction. That is, we explicitly calculate the inverse image $\gamma_{0}$ of the unit, the trivial (chordless) chord diagram, under the Kontsevich isomorphism. It is explicitly described as combinations of two-bridge knots (Theorem 3.22). We also show that the invariant space of proalgebraic knots under the $G T(\mathbb{K})$-action is one-dimensional generated by the element $\gamma_{0}$ (Theorem 3.27).

The following lemma is a knot analogue of Proposition 3.6.

Lemma 3.18. For any $\sigma \in G R T(\mathbb{K})$

$$
\rho_{0}(\sigma)(D)=\theta_{0}^{\Delta(\sigma)}(D)
$$

holds for all $D \in \widehat{\mathbb{K}[\mathcal{I K}]}$.

Proof. It can be proven in a completely same way to the proof of Proposition 3.6.

We may say that the following is an analogue of Theorem 3.10 and 3.14 for proalgebraic knots.

Proposition 3.19. (1) The $G R T(\mathbb{K})$-action $\rho_{0}$ constructed in (2.20) on the algebra $\widehat{\mathbb{K}[\mathcal{I K}]}$ of infinitesimal knots actually factors through $\mathbb{G}_{m}(\mathbb{K})\left(=\mathbb{K}^{\times}\right)$-action. Namely the kernel of the action is its unipotent part $G R T_{1}(\mathbb{K})$. 
(2) The $G T(\mathbb{K})$-action $\rho_{0}$ constructed in $(2.12)$ on the algebra $\widehat{\mathbb{K}[\mathcal{K}]}$ of proalgebraic knots actually factors through $\mathbb{G}_{m}(\mathbb{K})\left(=\mathbb{K}^{\times}\right)$-action. Namely the kernel of the action is its unipotent part $G T_{1}(\mathbb{K})$.

Proof. (1) It is obtained from a combination of (3.15) and (3.6) because we have $s(\bigcirc)=t(\circlearrowleft)=\emptyset$.

(2) The proof is almost same to the proof of Theorem 3.14. It can be derived from the above claim in this proposition and Proposition 2.26.

As a corollary of the above proposition, we obtain a non-trivial decomposition of knots below.

Corollary 3.20. Each oriented knot $K$ admits a canonical decomposition

$$
K=K_{0}+K_{1}+K_{2}+\cdots
$$

in $\widehat{\mathbb{K}[\mathcal{K}]}$ such that

$$
\sigma\left(K_{m}\right)=\lambda^{m} \cdot K_{m}
$$

holds for $\sigma=(\lambda, f) \in G T(\mathbb{K})$ and $m \geqslant 0$.

Proof. It is a direct corollary of Proposition 3.19 (2). By the representation theory of $\mathbb{G}_{m}$, the completed vector space $\widehat{\mathbb{K}[\mathcal{K}]}$ is decomposed into the product of eigenspaces $V_{a}(a \in \mathbb{Z})$ where $\mathbb{G}_{m}$ acts as a multiplication of $a$-th power:

$$
\widehat{\mathbb{K}[\mathcal{K}]}=\prod_{i \geqslant 0} V_{i}
$$

By our construction, the decomposition is compatible its filtration $\{\mathcal{K}\}_{N=0}^{\infty}$, i.e. $\prod_{i \geqslant N} V_{i}=\mathcal{K}_{N}$. We also have $\operatorname{dim} V_{a}<\infty$ for all $a \in \mathbb{Z}$ and actually $\operatorname{dim} V_{a}=0$ for $a<0$. Thus we get the claim.

Another Proof. Again by the representation theory of $\mathbb{G}_{m}$, Proposition 3.19.(1) implies that the completed vector space $\widehat{\mathbb{K}[\mathcal{I K}]}$, hence $\widehat{\mathcal{C D}}(\circlearrowleft)$, is decomposed into the product of eigenspaces $W_{a}(a \in \mathbb{Z})$ where $\mathbb{G}_{m}$ acts as a multiplication of $a$-th power:

$$
\widehat{\mathcal{C D}}(\circlearrowleft)=\prod_{i \geqslant 0} W_{i}
$$

Since the $\mathbb{G}_{m}$-action is compatible with the grading $\widehat{\mathcal{C D}}(\circlearrowleft)=\widehat{\oplus}_{m=0}^{\infty} \mathcal{C D}^{m}(\circlearrowleft)$ where $\mathcal{C D}^{m}(\bigcirc)$ is the $\mathbb{K}$-linear space spanned by the chord diagrams with $m$-chords, we have

$$
W_{-m}=\mathcal{C D}^{m}(\circlearrowleft)
$$

for $m \geqslant 0$. Thus $\operatorname{dim} W_{a}<\infty$ for all $a \in \mathbb{Z}$ and actually $\operatorname{dim} W_{a}=0$ for $a>0$. Let $p=(1, \varphi) \in M_{1}(\mathbb{K})$. Then we have an isomorphism

$$
r_{p}: G T(\mathbb{K}) \simeq G R T(\mathbb{K})
$$

by Proposition 1.18 and an isomorphism

$$
\rho_{0}(p): \widehat{\mathbb{K}[\mathcal{K}]} \simeq \widehat{\mathcal{C D}}(\circlearrowleft)
$$

by Proposition 2.25. (4). By using Proposition 2.26, we can show that

$$
V_{a}:=\rho_{0}(p)^{-1}\left(W_{-a}\right)
$$


is the eigenspace where $\mathbb{G}_{m}$ acts as a multiplication of $a$-th power and actually the space is invariant under any choice of $p=(1, \varphi) \in M_{1}(\mathbb{K})$.

We note that in the decomposition (3.17), we have $\operatorname{dim} V_{m}=\operatorname{dim} \mathcal{C D}^{m}(\bigcirc)$. for all $m \geqslant 0$.

Remark 3.21. Let $\iota_{0}=(-1,1) \in G T(\mathbb{K})$. Since it is an involution, the space $\widehat{\mathbb{K}[\mathcal{K}]}$ is divided into two eigenspaces $V_{+}$and $V_{-}$where the action of $\iota_{0}$ is given by the multiplication by 1 and -1 respectively. So an each oriented knot $K$ is decomposed as

$$
K=K_{+}+K_{-} \in \widehat{\mathbb{K}[\mathcal{K}]} \quad \text { with } \quad K_{ \pm} \in V_{ \pm} .
$$

Since $\iota_{0}(K)$ is nothing but the mirror image $\bar{K}$ of $K$, we have $K_{+}=\frac{1}{2}(K+\bar{K})$ and $K_{-}=\frac{1}{2}(K-\bar{K})$. We note that in terms of the decomposition (3.16) they are expressed as

$$
K_{+}=\sum_{i \geqslant 0} K_{2 i} \quad \text { and } \quad K_{-}=\sum_{i \geqslant 0} K_{2 i+1} .
$$

Our first theorem in this subsection is an explicit presentation of the proalgebraic knot whose Kontsevich invariant is trivial. That is, we explicitly calculate the inverse image of the unit, the trivial (chordless) chord diagram, under the Kontsevich isomorphism $I: \widehat{\mathbb{C}[\mathcal{K}]} \simeq \widehat{\mathcal{C D}}(\bigcirc)$ given in (2.33).

Theorem 3.22. The inverse image $I^{-1}(e)$ of the unit $e \in \widehat{\mathcal{C D}}(\circlearrowleft)(\simeq \widehat{\mathbb{K}[\mathcal{I K}]})$, under Kontsevich's isomorphism I is explicitly given by

$$
\gamma_{0}:=\bigcirc-c_{0}+c_{0} \sharp c_{0}-c_{0} \sharp c_{0} \sharp c_{0}+c_{0} \sharp c_{0} \sharp c_{0} \sharp c_{0}-\cdots \in \widehat{\mathbb{C}[\mathcal{K}]}
$$

where $\sharp$ is the connected sum and $c_{0} \in \widehat{\mathbb{C}[\mathcal{K}]}$ is given below (see also Figure 3.5):

$$
\begin{aligned}
c_{0}:= & \sum_{\substack{m, k_{1}, \ldots, k_{m} \in \mathbb{N} \\
k_{m}>1}}(-1)^{m} \frac{\zeta^{\operatorname{inv}}\left(k_{1}, \ldots, k_{m}\right)}{(2 \pi \sqrt{-1})^{k_{1}+\cdots+k_{m}}} \cdot\left\{a_{0,0}^{\curvearrowleft} \cdot a_{2,0}^{\downarrow \uparrow \uparrow} \cdot\left(\log \sigma_{2}^{2}\right)^{k_{m}-1} \cdot\left(\log \sigma_{3}^{2}\right) .\right. \\
& \left.\left(\log \sigma_{2}^{2}\right)^{k_{m-1}-1} \cdot\left(\log \sigma_{3}^{2}\right) \cdots \cdots\left(\log \sigma_{2}^{2}\right)^{k_{1}-1} \cdot\left(\log \sigma_{3}^{2}\right) \cdot c_{1,1}^{\downarrow \uparrow \uparrow} \cdot c_{0,0}\right\} .
\end{aligned}
$$

Here we define

$$
\log \sigma_{i}^{2}=-\sum_{k=1}^{\infty} \frac{1}{k}\left\{1-\sigma_{i}^{2}\right\}^{k} \in \widehat{\mathbb{C}\left[P_{4}\right]}
$$

for $i=2,3$. And we define the inversed $M Z V \zeta^{\text {inv }}\left(k_{1}, \ldots, k_{m}\right)$ to be the coefficient of $A^{k_{m}-1} B \cdots A^{k_{1}-1} B$ multiplied by $(-1)^{m}$ in the inversed KZ-associator $\Phi_{\mathrm{KZ}}^{\mathrm{inv}}(A, B) \in \mathbb{R}\langle\langle A, B\rangle\rangle$, which is the inverse of the $\mathrm{KZ}$-associator $\Phi_{\mathrm{KZ}}(A, B)$ in (1.15) with respect to the multiplication (1.11). Namely

$$
\begin{gathered}
\Phi_{\mathrm{KZ}}^{\mathrm{inv}}(A, B)=: 1+\sum_{\substack{m, k_{1}, \ldots, k_{m} \in \mathbb{N} \\
k_{m}>1}}(-1)^{m} \zeta^{\mathrm{inv}}\left(k_{1}, \cdots, k_{m}\right) A^{k_{m}-1} B \cdots A^{k_{1}-1} B \\
+ \text { (other terms) }
\end{gathered}
$$

where $\Phi_{\mathrm{KZ}}^{\mathrm{inv}}(A, B)$ is the series uniquely defined by

$$
\Phi_{\mathrm{KZ}}^{\mathrm{inv}}\left(\Phi_{\mathrm{KZ}}(A, B) \cdot A \cdot \Phi_{\mathrm{KZ}}(A, B)^{-1}, B\right)=\Phi_{\mathrm{KZ}}(A, B)^{-1} .
$$




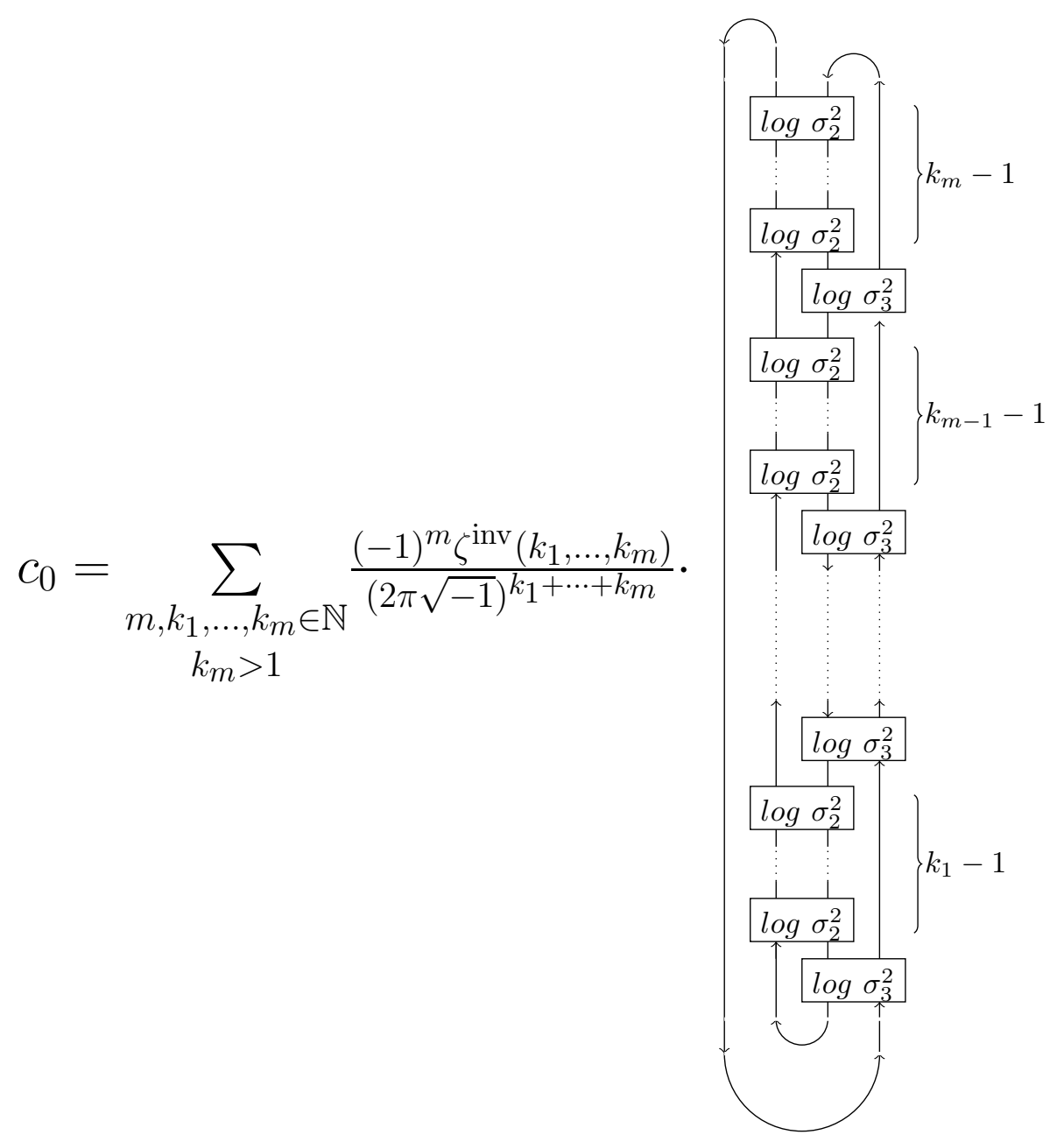

FiguRE 3.5. $c_{0}$

The inversed MZV's with small depth are calculated in Example 3.24 and the first two terms of $\gamma_{0}$ is calculated in Example 3.25 .

Proof. By our construction, the degree 0-part of the image $I(\circlearrowleft)$ of the trivial knot (unknot) O under the Kontsevich isomorphism $I$ given in (2.33) is the trivial (chordless) chord diagram $e$. Therefore on the decomposition $K=K_{0}+K_{1}+\cdots$ in (3.16) for $K=O$, we have

$$
K_{0}=I^{-1}(e)
$$

by (3.20). To calculate $K_{0}$, we take $\mathbb{K}$ to be the polynomial algebra $\mathbb{C}\left[T^{ \pm}\right]$generated by $T$ and $T^{-1}$ and take $p \in M_{1}(\mathbb{C})$ to be a specific element $p_{\mathrm{KZ}}=\left(1, \varphi_{\mathrm{KZ}}\right) \in$ $M(\mathbb{C})$ with $\varphi_{\mathrm{KZ}}=\Phi_{\mathrm{KZ}}\left(\frac{1}{2 \pi \sqrt{-1}} A, \frac{1}{2 \pi \sqrt{-1}} B\right)$. We have $\left(T^{-1}, 1\right) \in G R T(\mathbb{K})$. By Proposition 1.18, we obtain a unique element $\left(T, f_{T}\right) \in G T(\mathbb{K}(T))$ satisfying

$$
\left(T^{-1}, 1\right) \circ\left(1, \varphi_{\mathrm{KZ}}\right)=\left(1, \varphi_{\mathrm{KZ}}\right) \circ\left(T, f_{T}\right) .
$$


It can be read as

$$
\varphi_{\mathrm{KZ}}(T A, T B)=f_{T}\left(\varphi_{\mathrm{KZ}} \cdot e^{A} \cdot \varphi_{\mathrm{KZ}}^{-1}, e^{B}\right) \cdot \varphi_{\mathrm{KZ}} .
$$

We get that $f_{T}$ belongs to $F_{2}(\mathbb{C}[T])$. So

$$
f_{T}\left(\exp \left\{\varphi_{\mathrm{KZ}} \cdot A \cdot \varphi_{\mathrm{KZ}}^{-1}\right\}, \exp \{B\}\right) \equiv \varphi_{\mathrm{KZ}}^{-1} \quad(\bmod T) .
$$

By replacing $A$ and $B$ by $2 \pi \sqrt{-1} A$ and $2 \pi \sqrt{-1} B$ respectively, we obtain

$$
f_{T}\left(e^{2 \pi \sqrt{-1} A}, e^{2 \pi \sqrt{-1} B}\right) \equiv \Phi_{\mathrm{KZ}}^{\mathrm{inv}} \quad(\bmod T) .
$$

It says that

$$
f_{T}\left(\sigma_{1}^{2}, \sigma_{2}^{2}\right) \equiv \Phi_{\mathrm{KZ}}^{\mathrm{inv}}\left(\frac{\log \sigma_{1}^{2}}{2 \pi \sqrt{-1}}, \frac{\log \sigma_{2}^{2}}{2 \pi \sqrt{-1}}\right) \quad(\bmod T),
$$

where precisely it means that

$$
\operatorname{red}\left(f_{T}\left(\sigma_{1}^{2}, \sigma_{2}^{2}\right)\right)=\operatorname{red}\left(\Phi_{\mathrm{KZ}}^{\mathrm{inv}}\left(\frac{\log \sigma_{1}^{2}}{2 \pi \sqrt{-1}}, \frac{\log \sigma_{2}^{2}}{2 \pi \sqrt{-1}}\right)\right)
$$

with the reduction map red : $F_{2}(\mathbb{C}[T]) \rightarrow F_{2}(\mathbb{C})$ caused by putting $T=0$. Therefore by Definition 2.9

$$
\begin{aligned}
& \Lambda_{f_{T}}^{\uparrow}=a_{1,0}^{\uparrow \curvearrowleft} \cdot f_{T}\left(\sigma_{1}^{2}, \sigma_{2}^{2}\right) \cdot \widetilde{c_{0,1}^{\uparrow}} \\
& \equiv a_{1,0}^{\uparrow \curvearrowleft} \cdot \Phi_{\mathrm{KZ}}^{\mathrm{inv}}\left(\frac{\log \sigma_{1}^{2}}{2 \pi \sqrt{-1}}, \frac{\log \sigma_{2}^{2}}{2 \pi \sqrt{-1}}\right) \cdot{\overrightarrow{c_{0,1}}}_{\uparrow}^{\sim}(\bmod T) \\
& =a_{1,0}^{\uparrow \curvearrowleft} \cdot c_{0,1}^{\sim \uparrow}+\sum_{\substack{m, k_{1}, \ldots, k_{m} \in \mathbb{N} \\
k_{m}>1}}(-1)^{m} \frac{\zeta^{\mathrm{inv}}\left(k_{1}, \ldots, k_{m}\right)}{(2 \pi \sqrt{-1})^{k_{1}+\cdots+k_{m}}} \cdot\left\{a_{1,0}^{\uparrow \uparrow} \cdot\left(\log \sigma_{1}^{2}\right)^{k_{m}-1} \cdot\left(\log \sigma_{2}^{2}\right)\right. \\
& \left.\cdot\left(\log \sigma_{1}^{2}\right)^{k_{m-1}-1} \cdot\left(\log \sigma_{2}^{2}\right) \cdots \cdots\left(\log \sigma_{1}^{2}\right)^{k_{1}-1} \cdot\left(\log \sigma_{2}^{2}\right) \cdot c_{0,1}^{\sim \uparrow}\right\} .
\end{aligned}
$$

The third equality follows from (3.24). Here we note that the non-admissible terms, the 'other terms' in (3.24), all vanish by (T6). By the isomorphism (2.5), we have

$$
\operatorname{cl}\left(\Lambda_{f_{T}}^{\uparrow}\right)=a_{0,0}^{\curvearrowleft} \cdot\left(\downarrow \otimes \Lambda_{f_{T}}^{\uparrow}\right) \cdot c_{0,0}^{\bigcup} \equiv \bigcirc+c_{0} \quad(\bmod T)
$$

(for $c_{0}$, see Figure 3.5). Whence, by taking inverse of both, we have

$$
\operatorname{cl}\left(\nu_{f_{T}}^{\uparrow}\right) \equiv \gamma_{0} \quad(\bmod T)
$$

(for $\gamma_{0}$, see (0.1)). We note that the infinite summation on the right hand side of (3.21) converges because $c_{0}^{\sharp n} \in \mathcal{K}_{n}$ for $n \geqslant 1$. By Corollary 3.20, $K_{0}$ is obtained by evaluating $\left(T, f_{T}\right)(\bigcirc)$ at $T=0$, i.e.

$$
\left(T, f_{T}\right)(\circlearrowleft) \equiv K_{0} \quad(\bmod T)
$$

Since $\bigcirc=a_{0,0}^{\curvearrowleft} \cdot c_{0,0}^{\curlyvee}$, the action on $\bigcirc$ is calculated to be

$$
\left(T, f_{T}\right)(\bigcirc)=a_{0,0}^{\curvearrowleft} \cdot\left(\downarrow \otimes \nu_{f_{T}}^{\uparrow}\right) \cdot c_{0,0}^{\bigcup}=\operatorname{cl}\left(\nu_{f_{T}}^{\uparrow}\right) .
$$

By (3.27)-(3.29), we have

$$
\gamma_{0}=K_{0}
$$

because both $\gamma_{0}$ and $K_{0}$ are independent of $T$. Finally we obtain

$$
\gamma_{0}=I^{-1}(e)
$$

by (3.26). 
It might be worthy to mention that our $c_{0}$ is given by a linear combination of two bridge knots and our $\gamma_{0}$ is given by a linear combination of connected sums of two bridge knots.

Remark 3.23. We remind that the image $I(O)$ of the unit $O$, the trivial knot, under the Kontsevich isomorphism $I$ was calculated in [BLT], which may be be regarded as a calculation in an opposite direction to our theorem.

By the definition of inversed MZV's, (3.24) and (3.25), they are given by polynomial combinations of MZV's.

Example 3.24. (1) For $n>1$,

$$
\zeta^{\mathrm{inv}}(n)=-\zeta(n)
$$

(2) For $a>0$ and $b>1$,

$$
\begin{aligned}
\zeta^{\mathrm{inv}}(a, b)= & \zeta(a) \zeta(b)-\zeta(a, b)+\sum_{i=0}^{a-2}(-1)^{i}\left(\begin{array}{c}
i+b-1 \\
i
\end{array}\right) \zeta(b+i) \zeta(a-i) \\
& +(-1)^{a} \sum_{j=0}^{b-2}\left(\begin{array}{c}
j+a-1 \\
j
\end{array}\right) \zeta(b-j) \zeta(a+j) .
\end{aligned}
$$

Here is a brief computation of our $\gamma_{0}$.

Example 3.25.

$$
\gamma_{0} \equiv \bigcirc-\frac{1}{24}\left\{\bigcirc-3_{1}^{l}\right\} \quad\left(\bmod \mathcal{K}_{4}\right) .
$$

Here $3_{1}^{l}$ stands for the left trefoil knot.

Proposition 3.26. $\gamma_{0} \in \widehat{\mathbb{Q}[\mathcal{K}]}$.

Proof. In the proof of above theorem, there is no specific reason to choose $\left(1, \varphi_{\mathrm{KZ}}\right) \in$ $M_{1}(\mathbb{C})$. Take $(\mu, \varphi) \in M(\mathbb{K})$ to be any associator with an expansion

$$
\varphi(A, B)=1+\sum_{\substack{m, k_{1}, \ldots, k_{m} \in \mathbb{N} \\ k_{m}>1}}(-1)^{m} c\left(k_{1}, \cdots, k_{m}\right) A^{k_{m}-1} B \cdots A^{k_{1}-1} B+\text { (regularized terms). }
$$

Then $(1, \varphi(A / \mu, B / \mu)) \in M_{1}(\mathbb{K})$. By using $(1, \varphi(A / \mu, B / \mu)) \in M_{1}(\mathbb{K})$ instead of $\left(1, \varphi_{\mathrm{KZ}}\right) \in M_{1}(\mathbb{C})$ in the proof of Theorem 3.22 , we obtain an explicit formula of $c_{0}$, which is nothing but the replacement of $2 \pi \sqrt{-1}$ by $\mu \in \mathbb{K}^{\times}$and $\zeta\left(k_{1}, \ldots, k_{m}\right)$ by $c\left(k_{1}, \ldots, k_{m}\right)$ in (3.22). Thus our claim is obtained by particularly taking a rational associator, an element of $M(\mathbb{Q})$, whose existence is guaranteed in $[\mathrm{Dr}$.

Our second theorem in this subsection is on the invariant subspace of $\widehat{\mathbb{K}[\mathcal{K}]}$ under our $G T(\mathbb{K})$-action (cf. (2.12)).

Theorem 3.27. (1) The invariant subspace $V_{0}$ of $\widehat{\mathbb{K}[\mathcal{K}]}$ under our $G T(\mathbb{K})$-action is 1-dimensional and actually generated by $\gamma_{0}$.

(2) On the decomposition $K=K_{0}+K_{1}+\cdots$ given in (3.16),

$$
K_{0}=\gamma_{0}
$$

holds for any oriented knot $K$. 
Proof. (1) Since $\mathcal{C D}^{0}(\circlearrowleft)$ is 1-dimensional generated by the trivial (chordless) chord diagram $e$, the $\mathbb{K}$-linear space $V_{0}$ in (3.17) is 1 -dimensional by (3.19) and (3.20). Thus it is enough to show that $\rho_{0}(p)^{-1}(e)=\gamma_{0}$ for a particular choice of $p=$ $(1, \varphi) \in M_{1}(\mathbb{K})$. It is obtained by Theorem 3.22 because $\rho_{0}(p)=I$ for $p=p_{\mathrm{KZ}}$ (actually it holds for any $p \in M_{1}(\mathbb{K})$ ).

(2) By our construction, the degree 0-part of the image $I(K)$ is always equal to $e \in \mathcal{C D}^{0}(\circlearrowleft)$. So $I\left(K_{0}\right)=e$, by (3.20). Then by the arguments given above, we get $K_{0}=\gamma_{0}$.

Though our knot $\gamma_{0}$ is not equal to the trivial knot, the unknot 0 , we may say that it is the 'trivial' knot in a particular sense.

Remark 3.28. We saw in Remark 2.30 particularly that the space $\widehat{\mathbb{K}[\mathcal{K}]}$ of proalgebraic knots carries a structure of a mixed Tate (pro-)motive over Spec $\mathbb{Z}$. But Proposition 3.19 tells that it falls to infinite direct sum of Tate motives $\mathbb{Q}(n)$ for $n \geqslant 0$. Our $\gamma_{0}$ is the generator of the degree 0 -part $V_{0}$. Finding a basis of the degree $n$-part $V_{n}$ for another $n$, such as the basis which is dual to the Vassiliev invariants listed in [CDM] Table 3.2, is worthy to calculate.

Acknowledgements. Part of the paper was written at Max Planck Institute for Mathematics. The author also thanks the institute for its hospitality. The referees' efforts to make this paper better is gratefully acknowledged. This work was supported by Grant-in-Aid for Young Scientists (A) 24684001.

\section{REFERENCES}

[AT1] Alekseev, A. and Torossian, C., Kontsevich deformation quantization and flat connections, Comm. Math. Phys. 300 (2010), no. 1, 47-64.

[AT2] Alekseev, A. and Torossian, C., The Kashiwara-Vergne conjecture and Drinfeld's associators, Ann. of Math. (2) 175 (2012), no. 2, 415-463.

[AET] Alekseev, A., Enriquez, B. and Torossian, C., Drinfeld associators, braid groups and explicit solutions of the Kashiwara-Vergne equations, Publ. Math. Inst. Hautes Études Sci. No. 112 (2010), 143-189.

[A] André, Y., Une introduction aux motifs (motifs purs, motifs mixtes, périodes), Panoramas et Synthèses, 17, Société Mathématique de France, Paris, 2004.

[Ba1] Bar-Natan, D., Vassiliev and quantum invariants of braids, The interface of knots and physics (San Francisco, CA, 1995), 129-144, Proc. Sympos. Appl. Math., 51, Amer. Math. Soc., Providence, RI, 1996.

[Ba2] Bar-Natan, D., Non-associative tangles, Geometric topology (Athens, GA, 1993), 139-183, AMS/IP Stud. Adv. Math., 2.1, Amer. Math. Soc., Providence, RI, 1997.

[Ba3] Bar-Natan, D., On associators and the Grothendieck-Teichmuller group. I, Selecta Math. (N.S.) 4 (1998), no. 2, 183-212.

[BLT] Bar-Natan, D., Le, T. T. Q. and Thurston, D., Two applications of elementary knot theory to Lie algebras and Vassiliev invariants, Geom. Topol. 7 (2003), 1-31

[Be] Belyı̆, G. V., Galois extensions of a maximal cyclotomic field, Izv. Akad. Nauk SSSR Ser. Mat. 43 (1979), no. 2, 267-276, 479.

[Br1] Brown, F., Mixed Tate Motives over Spec(Z), Annals of Math., volume 175, no. 2 (2012), 949-976.

[Br2] Brown, F., Single-valued periods and multiple zeta values, preprint, arXiv:1309.5309

[C] Cartier, P., Construction combinatoire des invariants de Vassiliev-Kontsevich des nœuds, C. R. Acad. Sci. Paris Ser. I Math. 316, no. 11 (1993), 1205-1210.

[CDM] Chmutov, S., Duzhin, S. and Mostovoy, J., Introduction to Vassiliev Knot Invariants, Cambridge University Press, 2012.

[De] Deligne, P., Le groupe fondamental de la droite projective moins trois points, Galois groups over $\mathbb{Q}$ (Berkeley, CA, 1987), 79-297, Math. S. Res. Inst. Publ., 16, Springer, New YorkBerlin, 1989. 
[DeG] and Goncharov, A.; Groupes fondamentaux motiviques de Tate mixte, Ann. Sci. Ecole Norm. Sup. (4) 38 (2005), no. 1, 1-56.

[Dr] Drinfel'd, V. G., On quasitriangular quasi-Hopf algebras and a group closely connected with $\operatorname{Gal}(\overline{\mathbb{Q}} / \mathbb{Q})$, Leningrad Math. J. 2 (1991), no. 4, 829-860.

[F1] Furusho, H., The multiple zeta value algebra and the stable derivation algebra, Publ. Res. Inst. Math. Sci. Vol 39. no 4. (2003). 695-720.

[F2] Furusho, H., p-adic multiple zeta values I-p-adic multiple polylogarithms and the p-adic KZ equation, Inv. Math. Vol 155, Number 2, 253-286(2004).

[F3] Furusho, H., Multiple zeta values and Grothendieck-Teichmüller groups, AMS Contemporary Math, Vol 416, (2006), 49-82.

[F4] Furusho, H., p-adic multiple zeta values II - tannakian interpretations, Amer. J. Math, Vol 129, No 4, (2007),1105-1144.

[F5] Furusho, H., Pentagon and hexagon equations, Annals of Math. Vol. 171 (2010), No. 1, 545-556.

[F6] Furusho, H., Galois action on knots I: Action of the absolute Galois group, Quantum Topol. 8 (2017), no. 2, 295-360.

[H] Hain, R., The Hodge de Rham theory of relative Malcev completion, Ann. Sci. École Norm. Sup. (4) 31, no. 1, 47-92, 1998.

[I] Ihara, Y., Braids, Galois groups, and some arithmetic functions, Proceedings of the International Congress of Mathematicians, Vol. I, II (Kyoto, 1990), 99-120, Math. Soc. Japan, Tokyo, 1991.

[JS] Joyal, A. and Street, R., Braided tensor categories, Adv. Math. 102 (1993), no. 1, 20-78.

[K] Kassel, C., Quantum groups, Graduate Texts in Mathematics,155. Springer-Verlag, New York, 1995.

[KRT] Kassel, C., Rosso, M. and Turaev, V., Quantum groups and knot invariants, Panoramas et Synthèses, 5, Société Mathématique de France, Paris, 1997.

[KT] Kassel, C. and Turaev, V., Chord diagram invariants of tangles and graphs, Duke Math. J. 92 (1998), no. 3, 497-552.

[Ko] Kontsevich, M., Vassiliev's knot invariants, I. M. Gel'fand Seminar, 137-150, Adv. Soviet Math.16, Part 2, Amer. Math. Soc., Providence, RI, 1993.

[LM1] Le, T. T. Q. and Murakami, J., The universal Vassiliev-Kontsevich invariant for framed oriented links, Compositio Math. 102 (1996), no. 1, 41-64.

[LM2] _ _ Kontsevich's integral for the Kauffman polynomial, Nagoya Math. J. 142 (1996), $39-65$.

[LS] Lochak, P. and Schneps, L., Every acyclotomic element of the profinite GrothendieckTeichmüller group is a twist, Rev. Roumaine Math. Pures Appl. 60 (2015), no. 2, 117-128.

[M] Majid, S. A quantum groups primer, London Mathematical Society Lecture Note Series, 292. Cambridge University Press, Cambridge, 2002.

[P] Piunikhin, S., Combinatorial expression for universal Vassiliev link invariant, Comm. Math. Phys. 168 (1995), no. 1, 1-22.

[RW] Rossi, C.A. and Willwacher, T.; P. Etingof's conjecture about Drinfeld associators, preprint, arXiv:1404.2047

[SW] Ševera, P. and Willwacher, T., Equivalence of formalities of the little discs operad, Duke Math. J. 160 (2011), no. 1, 175-206.

[T] Turaev, V. G., Operator invariants of tangles, and R-matrices, Izv. Akad. Nauk SSSR Ser. Mat. 53 (1989), no. 5, 1073-1107, 1135; translation in Math. USSR-Izv. 35 (1990), no. 2, 411-444

[U] Ünver, S., Drinfel'd-Ihara relations for p-adic multi-zeta values, J. Number Theory 133 (2013), no. 5, 1435-1483.

Graduate School of Mathematics, Nagoya University, Chikusa-ku, Furo-cho, Nagoya, 464-8602, JAPAN

E-mail address: furusho@math.nagoya-u.ac.jp 